\title{
Faint Lines in the Arc Spectrum of Iron ( $\mathrm{Fe} \mathrm{I})$
}

\author{
Carl C. Kiess, Vera C. Rubin, and Charlotte E. Moore
}

(September 9, 1960)

\begin{abstract}
A search for new faint iron lines has been made on spectrograms taken with an arcin-air as source. The range of observations is from 2102 to $8679 \mathrm{~A}$. The reciprocal dispersion of the spectrographs used for the various spectral regions varies from $1 \mathrm{~A} / \mathrm{mm}$ to $3 \mathrm{~A} / \mathrm{mm}$.

Twelve new energy levels have been found, resulting in a total of 121 classified lines. A table containing 698 classified lines includes many lines whose wavelengths had been predicted as combinations among the known energy levels, and found in the solar spectrum in earlier work. Their reality has been confirmed in the present work.

A list containing 1,102 newly measured unclassified lines is included. Many of the lines listed in the tables have been reported by other observers with varying degrees of accuracy. All such reference sources are indicated in the tables. As a result of the new measurements, these lines may safely be attributed to Fe I.

A comparison of the new lines with the solar spectrum has resulted in the identification of 306 solar lines of Fe I unblended, and of 85 as blends to which Fe I is a contributor.
\end{abstract}

When the analysis of Fe I was carried out in $1944,\left[{ }^{1}\right],{ }^{1}$ it was recognized that the laboratory observations - were far from complete. From the known atomic energy levels, wavelengths were calculated for missing members of multiplets. The presence in the solar spectrum of almost all known lines of neutral iron indicated that a search for these "predicted" lines might be rewarding. The predicted lines were graded into three classes, good, fair, and poor, the grades being assigned roughly on the likelihood of the transition according to the rules of the quantum theory, the agreement between calculated and solar wavelengths, and the solar intensity with respect to known lines in the respective multiplets. The lines in the categories "good" and "fair" were published in Table C of the 1944 Monograph [1].

A search for faint iron lines has been made on spectrograms taken with an arc-in-air as source, and with exposures long enough to reveal fainter lines than have been recorded previously. The range of observations is from 2102 to $8679 \mathrm{~A}$. The electrodes used in the arcs were prepared from the purest iron obtainable at the time. The observations were made by the senior author (CCK) with the spectrographs at NBS. The spectrograms that have been measured are listed in table 1. The letters indicate the type of instrument used to cover the various spectral regions, namely:

$\mathrm{H}$ Prism: reciprocal dispersion $1 \mathrm{~A} / \mathrm{mm}$ to $1 / 3$ $\mathrm{A} / \mathrm{mm}$.

$\mathrm{X}$ Grating: Wood, $21 \mathrm{ft}$. concave grating 30,000 lines per inch, reciprocal dispersion $1 \mathrm{~A} / \mathrm{mm}$ in the second order.

$\mathrm{R}$ Grating: Rowland, $21 \mathrm{ft}$. concave grating 20,000 lines per inch, reciprocal dispersion $3 \mathrm{~A} / \mathrm{mm}$.

${ }^{1}$ Figures in brackets indicate the literature references on page 3.
TABLE 1. List of spectrograms

\begin{tabular}{|c|c|c|c|}
\hline Plate & $\begin{array}{c}\text { Region } \\
\text { A }\end{array}$ & Plate & $\begin{array}{c}\text { Region } \\
\mathrm{A}\end{array}$ \\
\hline $\begin{array}{lr}\mathrm{H} & 53 \\
\mathrm{H} & 55 \\
\mathrm{H} & 41 \\
\mathrm{H} & 42 \\
\mathrm{H} & 27 \\
\mathrm{H} & 26 \\
\mathrm{H} & 13 \\
\mathrm{H} & 1 \\
\mathrm{H} & 65 \\
\mathrm{H} & 76 \\
\mathrm{H} & 86 \\
\mathrm{H} & 96\end{array}$ & $\begin{array}{l}2102 \text { to } 2178 \\
2103 \text { to } 2178 \\
2136 \text { to } 2224 \\
2138 \text { to } 2231 \\
2184 \text { to } 2301 \\
2184 \text { to } 2303 \\
2260 \text { to } 2368 \\
2327 \text { to } 2474 \\
2404 \text { to } 2582 \\
2488 \text { to } 2714 \\
2611 \text { to } 2851 \\
2767 \text { to } 3083\end{array}$ & $\begin{array}{ll}\mathrm{X} & 242 \\
\mathrm{X} & 441 \\
\mathrm{X} & 546 \\
\mathrm{X} & 430 \\
\mathrm{X} & 436 \\
\mathrm{X} & 244 \\
\mathrm{X} & 132 \\
\mathrm{X} & 275 \\
\mathrm{X} & 303 \\
\mathrm{X} & 630 \\
\mathrm{X} & 453 \\
\mathrm{X} & 304 \\
\mathrm{X} & 452 \\
\mathrm{R} & 640 \\
\mathrm{R} & 638\end{array}$ & $\begin{array}{l}3007 \text { to } 3773 \\
3090 \text { to } 3820 \\
3348 \text { to } 3631 \\
3358 \text { to } 4482 \\
3581 \text { to } 3820 \\
3614 \text { to } 4294 \\
4250 \text { to } 4635 \\
4280 \text { to } 4425 \\
4400 \text { to } 6625 \\
4632 \text { to } 5216 \\
4752 \text { to } 6421 \\
5145 \text { to } 6253 \\
6885 \text { to } 8643 \\
6608 \text { to } 7945 \\
7832 \text { to } 8679\end{array}$ \\
\hline
\end{tabular}

In order to obtain at least two exposures over the entire range it has been necessary to fill in gaps by using $\mathrm{Fe}$ comparison spectra from miscellaneous spectrograms in the collection at the Georgetown College Observatory. These were taken with the Rowland grating described above. A serious attempt has been made to eliminate all possible impurities from the spectra.

The present work has been carried out to confirm the predicted lines attributed to $\mathrm{Fe}_{\mathrm{I}}$ in the solar spectrum, and to extend the identifications of FeI in the sun. It was hoped, also, that the analysis could be extended by additional observations. A search has been made among the new unclassified lines for levels combining with the known low terms of Fe I. Twelve new levels have been found, of which three are subject to some question. These levels are listed in table 2 with their respective $J$-values entered in column 2 . They account for a 
TABLE 2. New odd levels of Fe I

\begin{tabular}{|c|c|c|c|}
\hline Level & $J$ & Level & $J$ \\
\hline $\begin{array}{l}4945 \% .36^{\circ} ? \\
5335 \% .53^{\circ} \\
53610.44^{\circ} \\
53733.51^{\circ} \\
53749.39^{\circ} \\
53784.74^{\circ}\end{array}$ & $\begin{array}{l}4 \\
3 \\
4 \\
3 \\
2 \\
3\end{array}$ & $\begin{array}{l}53881.91^{\circ} \\
54289.09^{\circ} \\
5435 \% .40^{\circ} \\
57565.35^{\circ} ? \\
60563.61^{\circ} \\
62081.27^{\circ} ?\end{array}$ & $\begin{array}{l}4 \\
3 \\
3 \\
3 \\
3 \\
2\end{array}$ \\
\hline
\end{tabular}

total of 121 classified lines. Observations with a more suitable source will be required to extend this study further. One serious disadvantage encountered here is the masking of faint lines by wings of stronger ones. An electrodeless lamp doubtless would excite many new faint lines, and thus make it possible to find additional terms and assign their configurations.

The classified lines are listed in table 3 . The wave lengths in column 1 are the mean values from the present work. They include early unpublished measurements by Burns and Kiess, as well as a number of more recent ones by various workers. In preparing the final lists no line has been included as real unless it has been measured on at least two exposures. Many have more than two measurements. The classifications are new except for those lines in Table $\mathrm{C}$ of the Monograph that have been confirmed in the present work, and those lines having notes (a) and (b).

Some wavelengths are in italics. These were included in the original Princeton line list as unclassified and have been used for combinations with the new levels in Table 2. The best available reference source has been adopted for these lines, as was done in the Monograph. They are not new lines, but are newly classified.

The total number of lines in table 3 is 698 . The lines are mostly faint. Estimated intensities on an arbitrary scale are given in column 2. Diffuse lines are indicated by " $n$ " and " $N$ ". The next two columns contain, respectively, the observed wave numbers and those calculated from the term combinations. The designations in column 5 are those used for Fe I in the 1944 Monograph [1] and in "Atomic Energy Levels" [2]. The multiplet numbers in column 6 are from the 1945 Princeton Multiplet Table [3]. New lines belonging to known multiplets have been assigned the appropriate multiplet rumber. The notes in column 7 explain the different categories of lines. Most of the classifications are either "New" or "Pred". The latter include lines from Table $\mathrm{C}$ of the Monograph, i.e., those predicted lines classed as "good" or "fair" for which a solar wavelength was used. Additional predicted lines are also included, as indicated by the note (b) in this column. Although the predicted Fe I lines were classified earlier, they are included because for the first time they have been confirmed from laboratory observations. A few lines have the note "SS". The solar wavelengths were previously used for these lines as preferable to various laboratory values.
The letters in the last column are the same ones used for reference sources in the Monograph line list. They refer to lines that are not new but are newly classified, to lines measured by earlier observers that needed additional confirmation, or to unpublished measurements from the Massachusetts Institute of Technology, kindly furnished by G. R. Harrison in 1942. References $\mathrm{Z}, \mathrm{BK}$, and $\mathrm{ZZ}$ have been added.

In 1934 a list of faint lines of Fe I was compiled by Burns and Kiess. It contains early unpublished measurements made by Burns at Bonn and possibly at the Allegheny Observatory, and, also, measurements by Kiess of "H" plates described above. Lines in tables 3 and 4 that are present in this early list are so indicated by "BK" in the last column.

Table 4 contains the newly measured unclassified lines of Fe I, 1102 in all. The four columns contain respectively, the wavelengths, estimated intensities, wave numbers, and a column headed "Notes and References". As before, diffuse lines are marked " $n$ " and " $N$ " in the intensity column. In the last column the letters denote the same reference sources as were used in the 1944 paper. The symbols and references are described at the end of table 3.

Table 5 contains the solar lines that have been newly identified as Fe I, from the present work. The total number is 391, of which 306 are unblended, and 85 blended in the solar spectrum. The lefthand part of the table contains the laboratory data, i.e., wavelength and intensity from tables 3 and 4 . The solar data are entered in the right-hand part of the table: wavelength, disk intensity, the difference between the solar and laboratory wavelength, $(\odot-$ lab.), and the solar identification. These data are from the current revision of the solar spectrum now in progress [5]. From $\lambda 2945$ to $\lambda 3164$ the disk intensities are eye estimates: those in the range $\lambda 2945$ to the $\lambda 3062$ are from the 1948 paper [6], from $\lambda 3062$ to $\lambda 3164$ they are from Rowland's Preliminary Table of Solar Spectrum Wavelengths, as quoted in the 1928 edition [4]. The estimated intensities are entered in brackets. From $\lambda 3164$ to longer waves Rowland's estimated intensities are replaced by equivalent widths measured by Minnaert and Houtgast at Utrecht [5]. Italics denote that the reduced equivalent width is the weighted mean of the Utrecht measurements and of those by other observers.

In the last column of table 5 a predominant contributor to a solar line that is a blend, is indicated by the symbol "\|l". A leading contributor has the symbol "". A dash is used in this column to show whether the contributors to a blend are on the shortor long-wave side of the solar line. For example, the solar line at $4424.072 \mathrm{~A}$ is identified as a blend of Fe I on the short-wave side and $\mathrm{Cr} 1$ on the longwave side. Approximately 50 more lines in the present lists, whose identification in the solar spectrum is more dubious, have been omitted from table 5 . 
In conclusion, it is a pleasure to express our gratitude to those who have assisted with this laborious program. Special thanks are due Misses Eva Novotny and Janet Rountree for their work in measuring the plates. Mrs. Isabel Murray has prepared the tabular data with meticulous care. The work could not have been brought to its present stage tof completion without special financial aid. The project has been carried in part by grants NR 046136 from the Office of Naval Research and G 8193 from the National Science Foundation to the Georgetown College Observatory. Both of these are gratefully acknowledged.

\section{References}

[1] H. N. Russell and C. E. Moore, Trans. Am. Phil. Soc. 34, pt. II, 113 to 179 (1944).

[2] C. E. Moore, NBS Circ. 467, Vol. II, 49 to 54 (1952).

[3] C. E. Moore, NBS Tech. Note 36, PB 151395 (1959).

[4] C. E. St. John, C. E. Moore, L. M. Ware, E. F. Adams, H. D. Babcock, Carnegie Inst. Wash. Publ. No. 396, Papers Mt. Wilson Obs. III (1928).

[5] M. Minnaert and C. E. Moore, Revised Solar Table in progress, Utrecht Obs. and NBS, 1960; M. Minnaert, Recherches Astron. Obs. Utrecht 15, 168 ( $\lambda 3164$ to $\lambda 8770),(1960$.

[6] H. D. Babcock, C. E. Moore, and M. F. Coffeen, Astroph. J. 107, 287 to 302, Contr. Mount Wilson Obs. No. 745 (1948).

TABLE 3. Classified faint lines of Fe I

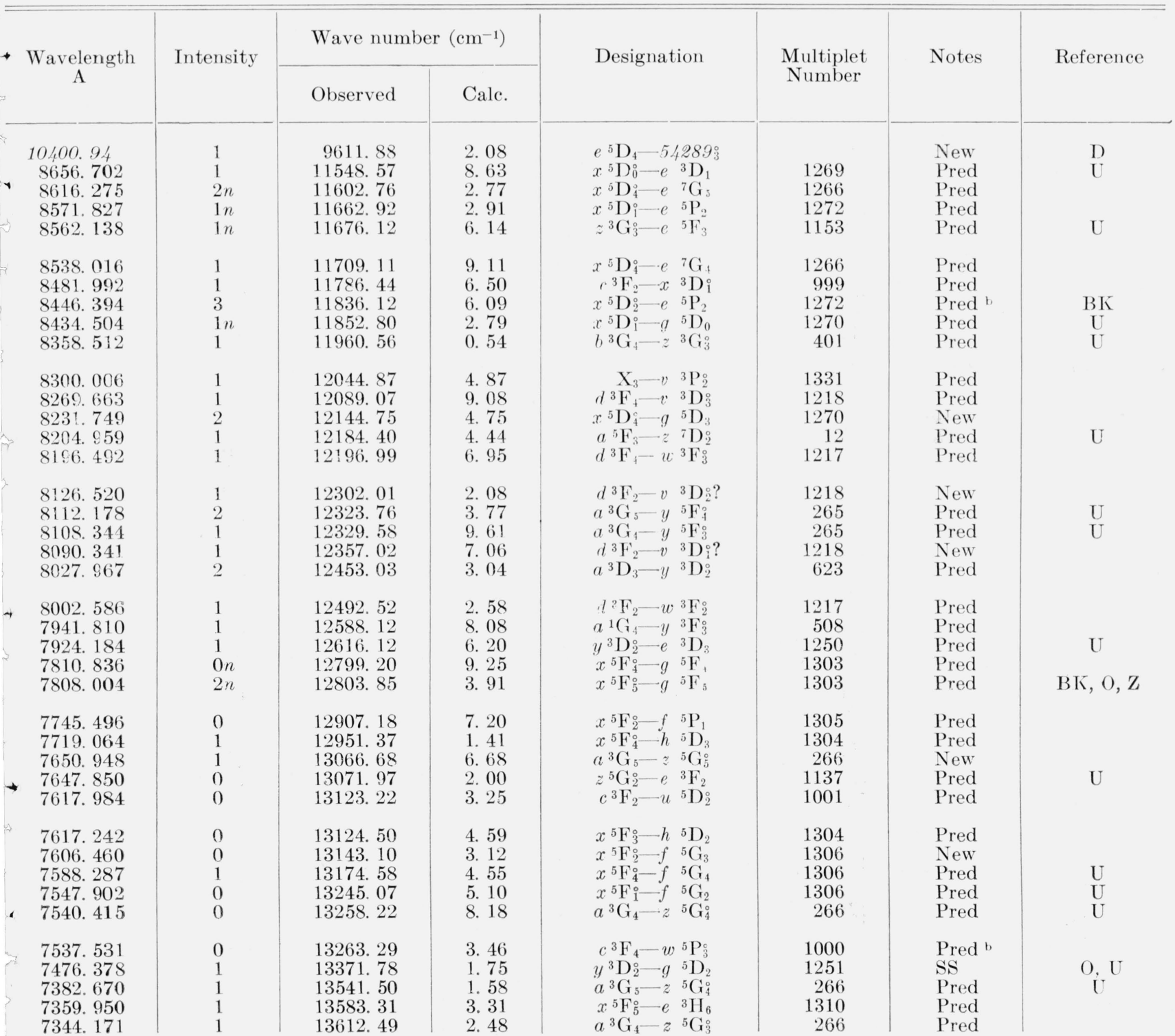


TABLE 3. Classified faint lines of Fe I-Continued

\begin{tabular}{|c|c|c|c|c|c|c|c|}
\hline \multirow{2}{*}{$\begin{array}{l}\text { Wavelength } \\
\text { A }\end{array}$} & \multirow{2}{*}{ Intensity } & \multicolumn{2}{|c|}{ Wave number $\left(\mathrm{cm}^{-1}\right)$} & \multirow{2}{*}{ Designation } & \multirow{2}{*}{$\begin{array}{l}\text { Multiplet } \\
\text { Number }\end{array}$} & \multirow{2}{*}{ Notes } & \multirow{2}{*}{ Reference } \\
\hline & & Observed & Calc. & & & & \\
\hline $\begin{array}{l}7330.148 \\
7317.402 \\
7316.752 \\
7315.595 \\
7300.532\end{array}$ & $\begin{array}{l}1 \\
1 \\
1 \\
1 \\
1 n\end{array}$ & $\begin{array}{l}13638.53 \\
13662.29 \\
13663.50 \\
13665.66 \\
13693.86\end{array}$ & $\begin{array}{l}\text { 8. } 52 \\
\text { 2. } 28 \\
\text { 3. } 47 \\
\text { 5. } 68 \\
\text { 3. } 76\end{array}$ & $\begin{array}{c}y^{5} \mathrm{P}_{\mathrm{i}}-f{ }^{7} \mathrm{D}_{1} \\
x^{5} \mathrm{D}_{\mathrm{i}}-f \\
{ }^{3} \mathrm{D}_{2} \\
a^{3} \mathrm{G}_{5}-z{ }^{3} \mathrm{G}_{5}^{\circ} \\
z^{3} \mathrm{P}_{0}^{0}-e \\
c^{5} \mathrm{~F}_{1} \\
c^{3} \mathrm{~F}_{3}-z{ }^{3} \mathrm{H}_{4}^{\circ}\end{array}$ & $\begin{array}{r}1187 \\
1278 \\
267 \\
1105 \\
1003\end{array}$ & $\begin{array}{l}\text { Pred } \\
\text { Pred } \\
\text { Pred } \\
\text { New } \\
\text { Pred }\end{array}$ & $\begin{array}{l}\mathrm{U} \\
\mathrm{U} \\
\mathrm{U}\end{array}$ \\
\hline $\begin{array}{l}7256.180 \\
7213.900 \\
7197.182 \\
7190.143 \\
7127.576\end{array}$ & $\begin{array}{l}1 n \\
0 \\
1 \\
1 \\
1 n\end{array}$ & $\begin{array}{l}13777.56 \\
13858.31 \\
13890.50 \\
13904.10 \\
14026.15\end{array}$ & $\begin{array}{l}\text { 7. } 67 \\
8.43 \\
0.51 \\
\text { 4. } 14 \\
6.16\end{array}$ & 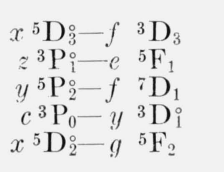 & $\begin{array}{r}1278 \\
1105 \\
1187 \\
463 \\
1273\end{array}$ & $\begin{array}{l}\text { SS } \\
\text { Pred } \\
\text { New } \\
\text { Pred } \\
\text { Pred }\end{array}$ & $\mathrm{U}$ \\
\hline $\begin{array}{l}7119.987 \\
7118.106 \\
7114.527 \\
7093.042 \\
7092.866\end{array}$ & $\begin{array}{l}1 \\
1 n \\
1 n \\
1 n \\
1 n\end{array}$ & $\begin{array}{l}14041.10 \\
14044.81 \\
14051.87 \\
14094.44 \\
14094.79\end{array}$ & $\begin{array}{l}\text { 1. } 06 \\
\text { 4. } 79 \\
\text { 1. } 82 \\
\text { 4. } 32 \\
\text { 4. } 85\end{array}$ & 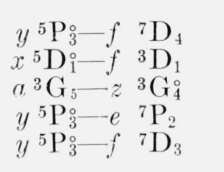 & $\begin{array}{r}1187 \\
1278 \\
267 \\
1189 \\
1187\end{array}$ & $\begin{array}{l}\text { Pred } \\
\text { Pred } \\
\text { Pred } \\
\text { Pred } \\
\text { New }\end{array}$ & ${ }_{\mathrm{U}}^{\mathrm{O}, \mathrm{U}}$ \\
\hline $\begin{array}{l}7072.832 \\
7069.571 \\
6936.501 \\
6847.605 \\
6785.754\end{array}$ & $\begin{array}{l}1 \\
0 \\
1 \\
1 \\
1\end{array}$ & $\begin{array}{l}14134.71 \\
14141.23 \\
14412.52 \\
14599.62 \\
14732.69\end{array}$ & $\begin{array}{l}4.75 \\
1.30 \\
2.57 \\
9.65 \\
2.69\end{array}$ & 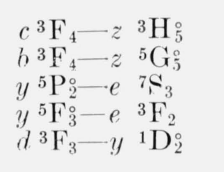 & $\begin{array}{r}1003 \\
205 \\
1196 \\
1078 \\
1226\end{array}$ & $\begin{array}{l}\text { Pred } \\
\text { SS } \\
\text { Pred } \\
\text { SS } \\
\text { Pred }\end{array}$ & $\begin{array}{l}\mathrm{U} \\
\mathrm{U} \\
\mathrm{U}\end{array}$ \\
\hline $\begin{array}{l}6745.969 \\
6704.513 \\
6565.700 \\
6249.667 \\
6028.344\end{array}$ & $\begin{array}{l}1 \\
1 \\
1 n \\
2 n \\
1\end{array}$ & $\begin{array}{l}14819.58 \\
14911.21 \\
15226.46 \\
15996.43 \\
16583.71\end{array}$ & $\begin{array}{l}9.61 \\
\text { 1. } 30 \\
6.39 \\
6.49 \\
\text { 3. } 68\end{array}$ & $\begin{array}{c}c^{3} \mathrm{~F}_{4}-w^{5} \mathrm{G}_{3}^{\circ} \\
y^{5} \mathrm{D}_{1}^{\circ}-e{ }^{3} \mathrm{~F}_{2} \\
\mathrm{X}_{3}-s{ }^{3} \mathrm{G}_{3}^{\circ} ? \\
z^{5} \mathrm{~F}_{4}^{\circ}-e{ }^{7} \mathrm{D}_{4} \\
c^{3} \mathrm{~F}_{4}-4945{ }^{\circ}{ }_{4}^{\circ} ?\end{array}$ & $\begin{array}{r}1005 \\
1052 \\
\\
685\end{array}$ & $\begin{array}{l}\text { Pred } \\
\text { SS } \\
\text { New } \\
\text { Pred } \\
\text { New }\end{array}$ & $\begin{array}{l}\mathrm{U} \\
\mathrm{U}\end{array}$ \\
\hline $\begin{array}{l}5905.030 \\
5899.094 \\
5738.248 \\
5473.171 \\
5470.105\end{array}$ & $\begin{array}{l}1 \\
2 \\
3 \\
2 \\
3\end{array}$ & $\begin{array}{l}16930.02 \\
16947.06 \\
17422.09 \\
18265.87 \\
18276.11\end{array}$ & $\begin{array}{l}0.07 \\
7.22 \\
2.17 \\
5.85 \\
6.06\end{array}$ & 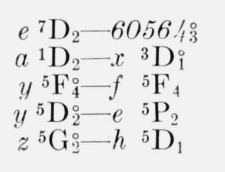 & $\begin{array}{r}738 \\
1084 \\
1064 \\
1144\end{array}$ & $\begin{array}{l}\text { New } \\
\text { New } \\
\text { Pred } \\
\text { Pred } \\
(a)\end{array}$ & $\begin{array}{c}\mathrm{U} \\
\mathrm{BK} \\
\mathrm{W}\end{array}$ \\
\hline $\begin{array}{l}5438.024 \\
5407.401 \\
5406.790 \\
5401.260 \\
5308.678\end{array}$ & $\begin{array}{l}3 \\
3 \\
3 \\
3 \\
1\end{array}$ & $\begin{array}{l}18383.92 \\
18488.03 \\
18490.12 \\
18509.05 \\
18831.84\end{array}$ & $\begin{array}{l}3.89 \\
8.17 \\
0.20 \\
9.04 \\
1.75\end{array}$ & 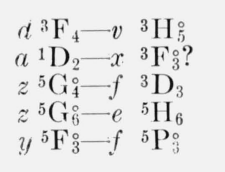 & $\begin{array}{l}1237 \\
1148 \\
1146 \\
1091\end{array}$ & $\begin{array}{l}\text { Pred } \\
\text { New } \\
\text { Pred } \\
\text { Pred } \\
\text { Pred }\end{array}$ & \\
\hline $\begin{array}{l}5305.397 \\
5300.403 \\
5297.142 \\
5245.717 \\
5238.246\end{array}$ & $\begin{array}{l}0 \\
1 \\
2 \\
3 \\
2\end{array}$ & $\begin{array}{l}18843.49 \\
18861.24 \\
18872.85 \\
19057.87 \\
19085.05\end{array}$ & $\begin{array}{l}\text { 3. } 44 \\
\text { 1. } 22 \\
\text { 2. } 81 \\
\text { 7. } 88 \\
\text { 5. } 04\end{array}$ & 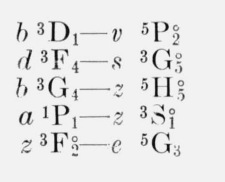 & $\begin{array}{r}877 \\
1240 \\
407 \\
715 \\
962\end{array}$ & $\begin{array}{l}\text { Pred b } \\
\text { Pred } \\
\text { New } \\
\text { Pred } \\
\text { Pred }\end{array}$ & \\
\hline $\begin{array}{l}5234.594 \\
5221.046 \\
5214.616 \\
5213.827 \\
5211.216\end{array}$ & $\begin{array}{l}3 \\
1 \\
3 \\
1 \\
2\end{array}$ & $\begin{array}{l}19098.36 \\
19147.92 \\
19171.53 \\
19174.43 \\
19184.04\end{array}$ & $\begin{array}{l}8.54 \\
\text { 7. } 92 \\
\text { 1. } 55 \\
\text { 4. } 53 \\
\text { 4. } 14\end{array}$ & $\begin{array}{l}b^{3} \mathrm{H}_{4}-x{ }^{5} \mathrm{G}_{i}^{\circ} \\
b{ }^{1} \mathrm{D}_{2}-53785^{\circ} \\
b^{1} \mathrm{D}_{2}-v{ }^{3} \mathrm{P}_{i} \\
z^{3} \mathrm{~F}_{3}-e \\
a^{5} \mathrm{G}_{4} \\
a^{1} \mathrm{P}_{1}-y{ }^{3} \mathrm{P}_{2}^{0}\end{array}$ & $\begin{array}{r}1131 \\
962 \\
716\end{array}$ & $\begin{array}{l}\text { New } \\
\text { New } \\
\text { New } \\
\text { Pred } \\
\text { New }\end{array}$ & $\underset{\mathrm{U}}{\mathrm{BK}}$ \\
\hline $\begin{array}{l}5209.883 \\
5207.960 \\
5205.372 \\
5204.953 \\
5197.942\end{array}$ & $\begin{array}{l}3 \\
3 \\
1 \\
2 \\
3\end{array}$ & $\begin{array}{l}19188.95 \\
19196.03 \\
19205.58 \\
19207.12 \\
19233.03\end{array}$ & $\begin{array}{l}8.91 \\
6.10 \\
\text { 5. } 60 \\
\text { 7. } 13 \\
\text { 3. } 09\end{array}$ & $\begin{array}{l}b^{3} \mathrm{H}_{6}-y{ }^{3} \mathrm{G}_{5}^{\circ} \\
b^{3} \mathrm{D}_{1}-x{ }^{3} \mathrm{P}_{i}^{\circ} \\
b^{3} \mathrm{H}_{4}-x \\
{ }^{5} \mathrm{G}_{4}^{\circ} \\
b^{3} \mathrm{G}_{4}-z \\
y^{5} \mathrm{H}_{3}^{\circ} \\
{ }^{5} \mathrm{~F}_{1}^{\circ}-f{ }^{5} \mathrm{P}_{1}\end{array}$ & $\begin{array}{r}584 \\
880 \\
\\
407 \\
1091\end{array}$ & $\begin{array}{l}\text { Pred } \\
\text { SS } \\
\text { New } \\
\text { Pred b } \\
\text { Pred }\end{array}$ & $\begin{array}{c}\mathrm{W}, \mathrm{ZZ} \\
\mathrm{U}\end{array}$ \\
\hline $\begin{array}{l}5174.703 \\
5157.156 \\
5156.599 \\
5149.492 \\
5146.322\end{array}$ & $\begin{array}{l}2 \\
3 n \\
2 \\
1 \\
3\end{array}$ & $\begin{array}{l}19319.40 \\
19385.13 \\
19387.23 \\
19413.98 \\
19425.94\end{array}$ & $\begin{array}{l}9.56 \\
5.25 \\
7.22 \\
\text { 3. } 96 \\
6.05\end{array}$ & $\begin{array}{c}c^{3} \mathrm{P}_{0}-w^{5} \mathrm{D}_{1}^{\circ} ? \\
\mathrm{X}_{2}-6056 \%_{i}^{\circ} \\
z^{3} \mathrm{~F}_{3}^{\circ}-e{ }^{7} \mathrm{~F}_{4} \\
z^{3} \mathrm{~F}_{3}^{\circ}-e{ }^{5} \mathrm{C}_{3} \\
z^{5} \mathrm{G}_{4}^{\circ}-f{ }^{3} \mathrm{~F}_{4}\end{array}$ & $\begin{array}{r}465 \\
\\
960 \\
962 \\
1150\end{array}$ & $\begin{array}{l}\text { New } \\
\text { New } \\
\text { New } \\
\text { New } \\
\text { Pred }\end{array}$ & $\begin{array}{l}\mathrm{L}, \mathrm{W} \\
\mathrm{BK}\end{array}$ \\
\hline
\end{tabular}


TABLE 3. Classified faint lines of Fe $\mathrm{I}$-Continued

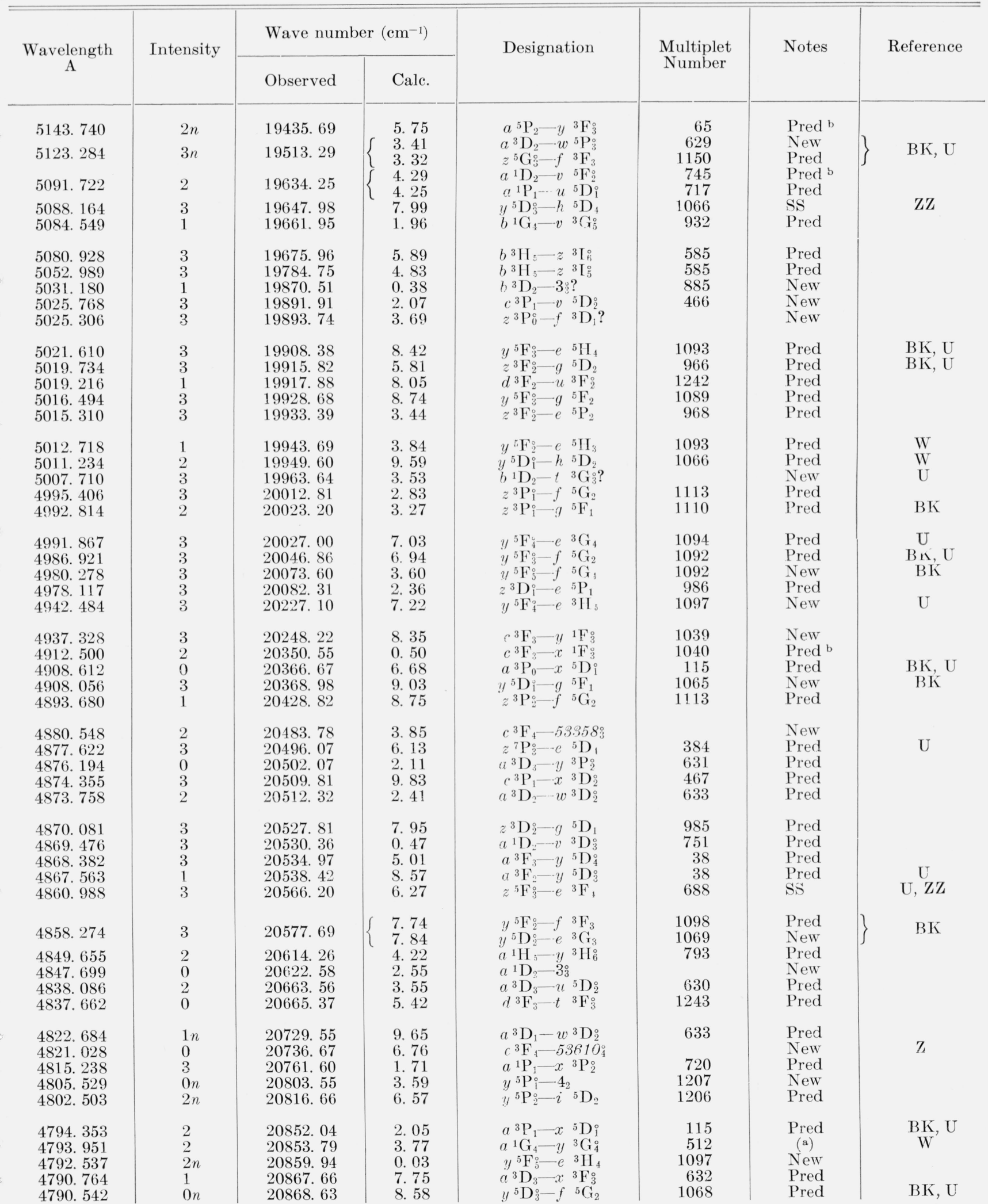


TABLE 3. Classified faint lines of Fe I-Continued

\begin{tabular}{|c|c|c|c|c|c|c|c|}
\hline \multirow{2}{*}{$\begin{array}{c}\text { Wavelength } \\
\text { A }\end{array}$} & \multirow{2}{*}{ Intensity } & \multicolumn{2}{|c|}{ Wave number $\left(\mathrm{cm}^{-1}\right)$} & \multirow{2}{*}{ Designation } & \multirow{2}{*}{$\begin{array}{l}\text { Multiplet } \\
\text { Number }\end{array}$} & \multirow{2}{*}{ Notes } & \multirow{2}{*}{ Reference } \\
\hline & & Observed & Calc. & & & & \\
\hline $\begin{array}{l}4782.789 \\
4780.789 \\
4773.496 \\
4760.050 \\
4758.689\end{array}$ & $\begin{array}{l}1 n \\
2 \\
0 \\
1 \\
1\end{array}$ & $\begin{array}{l}20902.46 \\
20911.20 \\
20943.15 \\
21002.31 \\
21008.32\end{array}$ & $\begin{array}{l}\text { 2. } 48 \\
\text { 1. } 11 \\
\text { 3. } 08 \\
\text { 2. } 23 \\
\text { 8. } 23\end{array}$ & $\begin{array}{l}b^{3} \mathrm{H}_{6}-z{ }^{3} \mathrm{H}_{3} \\
a^{3} \mathrm{D}_{3}-w{ }^{3} \mathrm{D}_{2} \\
b^{3} \mathrm{G}_{3}-x{ }^{3} \mathrm{D}_{2} \\
z^{7} \mathrm{P}_{2}-e \\
c^{5} \mathrm{D}_{1} \\
c^{3} \mathrm{~F}_{4}-53882_{4}^{8}\end{array}$ & $\begin{array}{l}588 \\
633 \\
408 \\
384\end{array}$ & $\begin{array}{l}\text { Pred } \\
\text { Pred } \\
\text { Pred b } \\
\text { Pred } \\
\text { New }\end{array}$ & $\mathrm{U}$ \\
\hline $\begin{array}{l}4749.580 \\
4744.615 \\
4742.920 \\
4718.410 \\
4716.816\end{array}$ & $\begin{array}{l}1 \\
1 n \\
1 \\
1 \\
0 n\end{array}$ & $\begin{array}{l}21048.61 \\
21070.63 \\
21078.16 \\
21187.65 \\
21194.81\end{array}$ & $\begin{array}{l}8.55 \\
0.55 \\
8.12 \\
7.57 \\
4.69\end{array}$ & $\begin{array}{l}a^{3} \mathrm{~F}_{2}-y{ }^{5} \mathrm{D}_{1}^{\circ} \\
a^{5} \mathrm{~F}_{2}-z{ }^{5} \mathrm{P}_{3} \\
y^{5} \mathrm{D}_{2}-e{ }^{3} \mathrm{P}_{2} \\
c^{3} \mathrm{~F}_{3}-t{ }^{3} \mathrm{G}_{3}^{\circ} \\
a^{3} \mathrm{D}_{3}-1_{2}^{\circ}\end{array}$ & $\begin{array}{r}38 \\
17 \\
1072 \\
1042 \\
634\end{array}$ & $\begin{array}{l}\text { New } \\
\text { Pred } \\
\text { Pred } \\
\text { New } \\
\text { Pred }\end{array}$ & $\mathrm{BK}, \mathrm{U}$ \\
\hline $\begin{array}{l}4702.926 \\
4690.367 \\
4685.036 \\
4677.572 \\
4674.651\end{array}$ & $\begin{array}{l}0 \\
1 \\
3 \\
2 n \\
2\end{array}$ & $\begin{array}{l}21257.41 \\
21314.33 \\
21338.58 \\
21372.63 \\
21385.98\end{array}$ & $\begin{array}{l}\text { 7. } 44 \\
\text { 4. } 31 \\
\text { 8. } 62 \\
\text { 2. } 58 \\
6.01\end{array}$ & $\begin{array}{l}b^{3} \mathrm{H}_{6}-w^{5} \mathrm{G}_{6}^{\circ} \\
a^{5} \mathrm{~F}_{1}-z{ }^{5} \mathrm{P}_{2}^{\circ} \\
b^{3} \mathrm{P}_{1}-w^{5} \mathrm{~F}_{2}^{\circ} \\
y^{5} \mathrm{D}_{3}-e{ }^{3} \mathrm{P}_{2} \\
a^{3} \mathrm{~F}_{3}-z{ }^{3} \mathrm{P}_{2}^{\circ}\end{array}$ & $\begin{array}{r}17 \\
347 \\
1072 \\
40\end{array}$ & $\begin{array}{l}\text { New } \\
\text { Pred } \\
\text { Pred } \\
\text { Pred } \\
\text { Pred }\end{array}$ & BK, $\mathrm{U}$ \\
\hline $\begin{array}{l}4665.522 \\
4653.446 \\
4642.624 \\
4636.672 \\
4634.170\end{array}$ & $\begin{array}{l}0 \\
1 \\
1 \\
1 \\
1\end{array}$ & $\begin{array}{l}21427.83 \\
21483.44 \\
21533.51 \\
21561.16 \\
21572.80\end{array}$ & $\begin{array}{l}\text { 7. } 68 \\
\text { 3. } 53 \\
\text { 3. } 72 \\
\text { 1. } 23 \\
2.82\end{array}$ & $\begin{array}{c}c^{3} \mathrm{~F}_{4}-13_{4}^{\circ} ? \\
b^{3} \mathrm{H}_{5}-x{ }^{3} \mathrm{G}_{5}^{\circ} \\
z^{5} \mathrm{~F}_{3}^{\circ}-e{ }^{3} \mathrm{~F}_{2} ? \\
a^{1} \mathrm{G}_{4}-z{ }^{3} \mathrm{I}_{5}^{\circ} \\
b^{3} \mathrm{P}_{2}-w^{5} \mathrm{D}_{1}^{\circ}\end{array}$ & $\begin{array}{r}1044 \\
591 \\
688 \\
513 \\
346\end{array}$ & $\begin{array}{l}\text { Pred } \\
\text { New } \\
\text { Pred } \\
\text { Pred } \\
\text { New }\end{array}$ & $B K$ \\
\hline $\begin{array}{l}4628.696 \\
4627.532 \\
4605.070 \\
4604.850 \\
4598.728\end{array}$ & $\begin{array}{l}0 \\
2 \\
0 \\
0 \\
1\end{array}$ & $\begin{array}{l}21598.31 \\
21603.74 \\
21709.12 \\
21710.15 \\
21739.05\end{array}$ & $\begin{array}{l}8.34 \\
3.69 \\
9.00 \\
0.15 \\
9.01\end{array}$ & $\begin{array}{c}z^{5} \mathrm{P}_{1}-e{ }^{7} \mathrm{~F}_{2} \\
b^{3} \mathrm{H}_{4}-w^{3} \mathrm{G}_{5} \\
b^{3} \mathrm{P}_{0}-v{ }^{5} \mathrm{D}_{1} \\
a^{1} \mathrm{I}_{6}-x{ }^{3} \mathrm{H}_{6}^{\circ} \\
z^{5} \mathrm{P}_{2}^{\circ}-e{ }^{7} \mathrm{~F}_{1}\end{array}$ & $\begin{array}{l}819 \\
593 \\
348 \\
846 \\
819\end{array}$ & $\begin{array}{l}\text { Pred } \\
\text { New } \\
\text { Pred } \\
\text { Pred } \\
\text { Pred }\end{array}$ & \\
\hline $\begin{array}{l}4591.502 \\
4583.726 \\
4572.849 \\
4571.448 \\
4561.426\end{array}$ & $\begin{array}{l}2 n \\
2 \\
2 \\
2 \\
2\end{array}$ & $\begin{array}{l}21773.26 \\
21810.20 \\
21862.08 \\
21868.78 \\
21916.83\end{array}$ & $\begin{array}{l}\text { 3. } 09 \\
0.27 \\
2.06 \\
8.83 \\
6.78\end{array}$ & $\begin{array}{l}a^{3} \mathrm{G}_{3}-w^{5} \mathrm{~F}_{4}^{\circ} ? \\
c^{3} \mathrm{P}_{0}-y{ }^{3} \mathrm{P}_{1}^{\circ} \\
z^{5} \mathrm{P}_{2}^{\circ}-e{ }^{7} \mathrm{~F}_{2} \\
z^{7} \mathrm{~F}_{2}^{\circ}-e{ }^{5} \mathrm{D}_{3} \\
a^{3} \mathrm{G}_{3}-w^{5} \mathrm{~F}_{3}^{\circ}\end{array}$ & $\begin{array}{l}472 \\
819 \\
319\end{array}$ & $\begin{array}{l}\text { New } \\
\text { Pred } \\
\text { (a) } \\
\text { Pred } \\
\text { New }\end{array}$ & $\begin{array}{c}\mathrm{U} \\
\mathrm{U}, \mathrm{W} \\
\mathrm{U} \\
\mathrm{U}\end{array}$ \\
\hline $\begin{array}{l}4546.477 \\
4541.319 \\
4540.651 \\
4533.953 \\
4520.240\end{array}$ & $\begin{array}{l}1 \\
2 \\
1 \\
1 \\
2\end{array}$ & $\begin{array}{l}21988.89 \\
22013.86 \\
22017.10 \\
22049.63 \\
22116.52\end{array}$ & $\begin{array}{l}\text { 8. } 96 \\
\text { 3. } 87 \\
\text { 7. } 07 \\
9.59 \\
6.52\end{array}$ & $\begin{array}{c}c^{3} \mathrm{~F}_{2}-w^{1} \mathrm{D}_{2}^{\circ} \\
a^{3} \mathrm{D}_{3}-v \\
b^{5} \mathrm{~F}_{2} \\
b^{3} \mathrm{G}_{4}-z{ }^{3} \mathrm{I}_{5}^{\circ} \\
b^{3} \mathrm{G}_{5}-x{ }^{5} \mathrm{G}_{1} \\
c^{3} \mathrm{P}_{1}-u^{5} \mathrm{D}_{2}^{\circ}\end{array}$ & $\begin{array}{r}1047 \\
640 \\
411 \\
410 \\
471\end{array}$ & $\begin{array}{l}\text { Pred } \\
\text { New } \\
\text { New } \\
\text { New } \\
(a, c)\end{array}$ & $\begin{array}{l}\text { BK, U } \\
\mathrm{U} \\
\mathrm{BK}, \mathrm{Z}\end{array}$ \\
\hline $\begin{array}{l}4518.583 \\
4516.265 \\
4515.146 \\
4513.713 \\
4507.232\end{array}$ & $\begin{array}{l}2 \\
1 \\
2 \\
1 \\
1\end{array}$ & $\begin{array}{l}22124.63 \\
22135.98 \\
22141.47 \\
22148.50 \\
22180.34\end{array}$ & $\begin{array}{l}\text { 4. } 67 \\
\text { 5. } 98 \\
\text { 1. } 37 \\
\text { 8. } 48 \\
0.48\end{array}$ & $\begin{array}{l}a^{5} \mathrm{P}_{1}-y{ }^{7} \mathrm{P}_{2}^{\circ} \\
z^{5} \mathrm{P}_{3}^{\circ}-e{ }^{7} \mathrm{~F}_{4} \\
z^{7} \mathrm{~F}_{2}^{\circ}-e \\
b^{5} \mathrm{D}_{2} \\
b^{3} \mathrm{~F}_{3}-y \\
c^{3} \mathrm{G}_{4}^{\circ} \\
{ }^{3} \mathrm{P}_{0}-w^{3} \mathrm{D}_{1}^{\circ}\end{array}$ & $\begin{array}{r}69 \\
819 \\
319 \\
213 \\
474\end{array}$ & $\begin{array}{l}\text { Pred } \\
\text { Pred } \\
\text { SS } \\
\text { Pred b } \\
\text { New c }\end{array}$ & $\underset{\mathrm{U}}{\mathrm{U}} \underset{\mathrm{ZZ}}{\mathrm{U}}$ \\
\hline $\begin{array}{l}4487.748 \\
4483.771 \\
4474.722 \\
4473.004\end{array}$ & $\begin{array}{l}1 \\
1 \\
1 \\
0\end{array}$ & $\begin{array}{l}22276.64 \\
22296.40 \\
22341.49 \\
22350.07\end{array}$ & $\begin{array}{l}\text { 6. } 68 \\
6.36 \\
\text { 1. } 51 \\
0.24\end{array}$ & $\begin{array}{c}b^{3} \mathrm{H}_{6}-z{ }^{1} \mathrm{H}_{5}^{\circ} \\
b^{3} \mathrm{D}_{3}-u^{3} \mathrm{G}_{4}^{\circ} \\
c^{3} \mathrm{~F}_{3}-w^{1} \mathrm{D}_{2}^{\circ} \\
z^{7} \mathrm{~F}_{1}-e{ }^{5} \mathrm{D}_{0} ?\end{array}$ & $\begin{array}{r}594 \\
898 \\
1047 \\
319\end{array}$ & $\begin{array}{l}\text { Pred } \\
\text { Pred } \\
\text { New } \\
\text { New }\end{array}$ & $\begin{array}{l}\mathrm{U} \\
\mathrm{U}\end{array}$ \\
\hline 4463.147 & 2 & 22399. 43 & $\begin{array}{l}\text { 9. } 46 \\
\text { 9. } 38\end{array}$ & $\begin{array}{l}c^{3} \mathrm{P}_{1}-u^{5} \mathrm{D}_{0}^{\circ} \\
b^{3} \mathrm{D}_{2}-7_{2}^{\circ}\end{array}$ & $\begin{array}{l}471 \\
901\end{array}$ & $\begin{array}{l}\text { Pred } \\
\text { Pred } b\end{array}$ & $\mathrm{U}$ \\
\hline $\begin{array}{l}4453.325 \\
4452.616 \\
4450.765 \\
4443.885 \\
4437.695\end{array}$ & $\begin{array}{l}2 \\
1 n \\
2 \\
1 \\
1\end{array}$ & $\begin{array}{l}22448.83 \\
22452.40 \\
22461.74 \\
22496.52 \\
22527.90\end{array}$ & $\begin{array}{l}9.03 \\
2.42 \\
\text { 1. } 75 \\
6.37 \\
7.90\end{array}$ & $\begin{array}{l}z^{5} \mathrm{D}_{1}^{\circ}-e{ }^{3} \mathrm{~F}_{2} \\
z^{3} \mathrm{~F}_{3}^{\circ}-g \\
{ }^{5} \mathrm{~F}_{2} \\
z^{3} \mathrm{~F}_{4}^{\circ}-f \\
b^{5} \mathrm{G}_{4} \\
b^{3} \mathrm{~F}_{4}-y{ }^{5} \mathrm{G}_{3}^{\circ} ? \\
a^{3} \mathrm{G}_{5}-w^{5} \mathrm{~F}_{5}^{\circ}\end{array}$ & $\begin{array}{l}555 \\
969 \\
972 \\
213\end{array}$ & $\begin{array}{l}\text { New } \\
\text { Pred } \\
\text { Pred } \\
\text { New } \\
\text { New }\end{array}$ & $\begin{array}{l}\mathrm{U} \\
\mathrm{U}\end{array}$ \\
\hline $\begin{array}{l}4428.554 \\
4419.790 \\
4419.076 \\
4417.334 \\
4407.230\end{array}$ & $\begin{array}{l}2 \\
0 \\
2 n \\
1 n \\
3\end{array}$ & $\begin{array}{l}22574.40 \\
22619.16 \\
22622.81 \\
22631.73 \\
22683.62\end{array}$ & $\begin{array}{l}\text { 4. } 34 \\
9.22 \\
2.64 \\
\text { 1. } 89 \\
\text { 3. } 62\end{array}$ & $\begin{array}{l}z^{3} \mathrm{~F}_{3}^{\circ}-e{ }^{3} \mathrm{G}_{3} \\
a^{3} \mathrm{D}_{2}-w^{3} \mathrm{~F}_{3}^{\circ} \\
z^{3} \mathrm{G}_{5}^{\circ}-g \\
z^{5} \mathrm{G}_{6}^{5} ? \\
z^{5} \mathrm{D}_{4}^{\circ}-e{ }^{3} \mathrm{~F}_{3} \\
z^{5} \mathrm{P}_{3}^{\circ}-e e^{3} \mathrm{D}_{2}\end{array}$ & $\begin{array}{r}973 \\
644 \\
1170 \\
555 \\
827\end{array}$ & $\begin{array}{l}\text { Pred } \\
\text { Pred } \\
\text { New } \\
\text { New } \\
\text { New }\end{array}$ & $\mathrm{U}$ \\
\hline
\end{tabular}


TABLE 3. Classified faint lines of Fe I-Continued

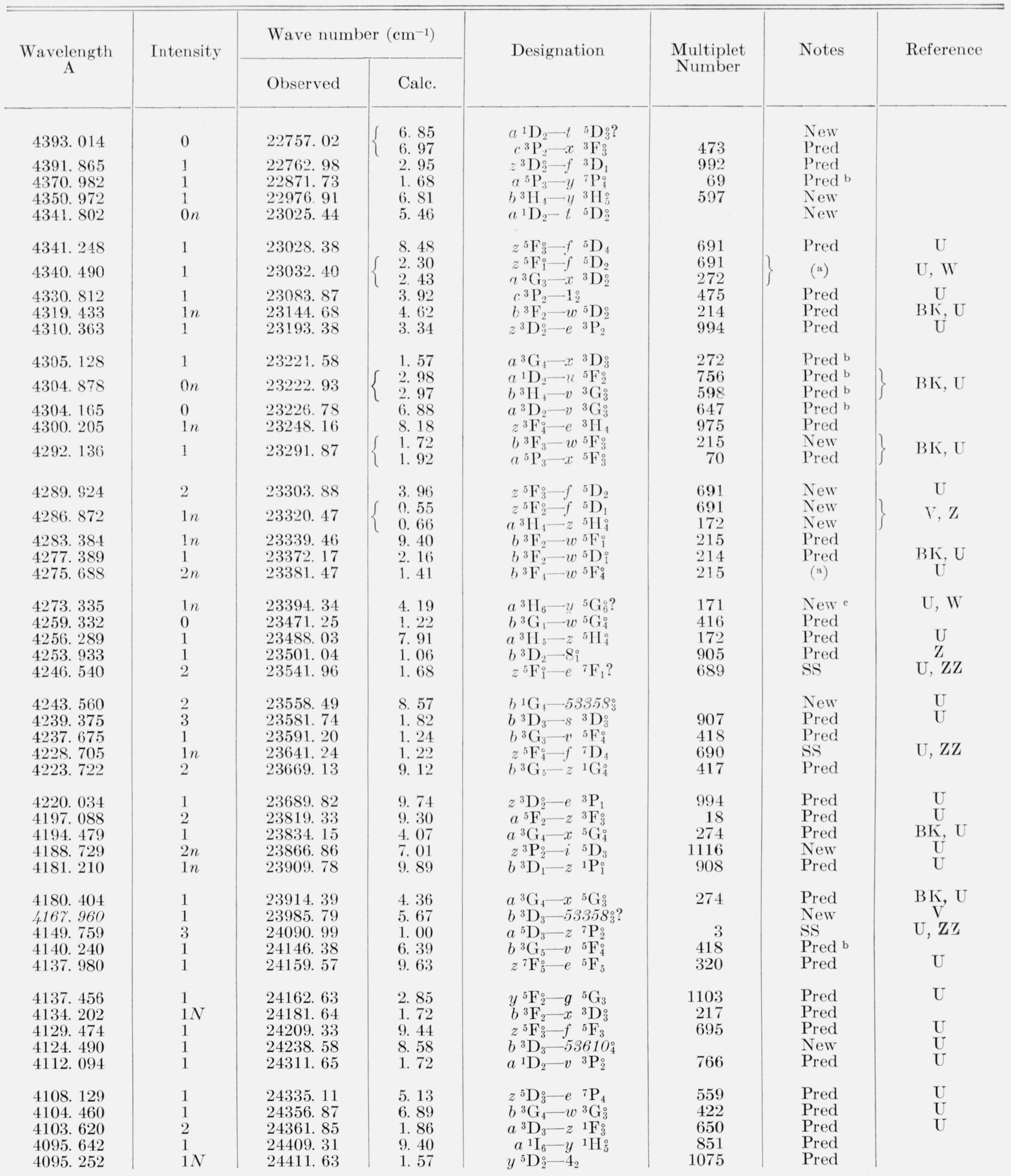


Table 3. Classified faint lines of Fe I-Continued

\begin{tabular}{|c|c|c|c|c|c|c|c|}
\hline \multirow{2}{*}{$\begin{array}{c}\text { Wavelength } \\
\text { A }\end{array}$} & \multirow{2}{*}{ Intensity } & \multicolumn{2}{|c|}{ Wave number $\left(\mathrm{cm}^{-1}\right)$} & \multirow{2}{*}{ Designation } & \multirow{2}{*}{$\begin{array}{l}\text { Multiplet } \\
\text { Number }\end{array}$} & \multirow{2}{*}{ Notes } & \multirow{2}{*}{ Reference } \\
\hline & & Observed & Calc. & & & & \\
\hline $\begin{array}{l}\text { 4092. } 287 \\
4090.326 \\
4079.214 \\
4078.822 \\
4070.422\end{array}$ & $\begin{array}{l}1 \\
1 \\
3 N \\
1 \\
0\end{array}$ & $\begin{array}{l}24429.32 \\
24441.03 \\
24507.61 \\
24509.96 \\
24560.54\end{array}$ & $\begin{array}{l}9.34 \\
1.00 \\
7.85 \\
0.05 \\
0.39\end{array}$ & $\begin{array}{c}b^{3} \mathrm{D}_{1}-53749_{2} \\
a^{3} \mathrm{~F}_{2}-y{ }^{5} \mathrm{P}_{1} \\
z^{5} \mathrm{~F}_{2}^{\circ}-e \\
{ }^{5} \mathrm{P}_{2} \\
b^{3} \mathrm{D}_{1}-5388^{\circ} 2_{4} \\
a^{1} \mathrm{G}_{4}-v{ }^{3} \mathrm{D}_{3}^{\circ} ?\end{array}$ & $\begin{array}{r}44 \\
700 \\
525\end{array}$ & $\begin{array}{l}\text { New } \\
\text { Pred } \\
\text { Pred } \\
\text { New } \\
\text { Pred b }\end{array}$ & $\begin{array}{c}\text { J } \\
\text { BK, U } \\
\text { BK, U } \\
\text { BK }\end{array}$ \\
\hline $\begin{array}{l}\text { 4070. } 010 \\
4057.654 \\
4036.370 \\
4031.727 \\
4022.212\end{array}$ & $\begin{array}{l}0 \\
1 \\
1 \\
2 \\
1 n\end{array}$ & $\begin{array}{l}24563.03 \\
24637.82 \\
24767.74 \\
24796.26 \\
24854.92\end{array}$ & $\begin{array}{l}\text { 3. } 03 \\
\text { 7. } 82 \\
\text { 7. } 78 \\
\text { 6. } 28 \\
\text { 4. } 93\end{array}$ & $\begin{array}{c}z^{7} \mathrm{~F}_{2}^{\circ}-e{ }^{5} \mathrm{~F}_{3} \\
a^{1} \mathrm{P}_{1}-t \\
{ }^{3} \mathrm{D}_{1}^{\circ} \\
a^{3} \mathrm{G}_{3}-w \\
b^{3} \mathrm{D}_{3}^{\circ} \mathrm{G}_{3}-v \\
{ }^{3} \mathrm{D}_{3}^{\circ} \\
b^{3} \mathrm{P}_{2}-w{ }^{5} \mathrm{G}_{3}^{\circ}\end{array}$ & $\begin{array}{l}320 \\
729 \\
279 \\
427 \\
360\end{array}$ & $\begin{array}{l}\text { New } \\
\text { Pred } \\
\text { Pred } \\
\text { Pred } \\
\text { New }\end{array}$ & $\begin{array}{c}\mathrm{BK} \\
\mathrm{U} \\
\mathrm{BK}, \mathrm{U} \\
\mathrm{UK}, \underset{\mathrm{U}}{\mathrm{U}}, \mathrm{W}\end{array}$ \\
\hline $\begin{array}{l}\text { 4001. } 212 \\
\text { 3998. } 743 \\
\text { 3996. } 779\end{array}$ & $\begin{array}{l}1 N \\
0 \\
1\end{array}$ & $\begin{array}{l}\text { 24985. } 36 \\
25000.79 \\
25013.08\end{array}$ & $\begin{array}{l}\text { 5. } 54 \\
0.62 \\
\text { 3. } 04\end{array}$ & $\begin{array}{l}b^{3} \mathrm{D}_{3}-54357^{\circ} \\
b^{3} \mathrm{D}_{2}-54357^{\circ} \\
y^{5} \mathrm{D}_{3}^{\circ}-g{ }^{5} \mathrm{G}_{4}\end{array}$ & 1074 & $\begin{array}{l}\text { New } \\
\text { New } \\
\text { Pred }\end{array}$ & $\begin{array}{c}\mathrm{BK}, \mathrm{U} \\
\mathrm{U}\end{array}$ \\
\hline 3996. 261 & $1 n$ & 25016. 32 & $\begin{array}{l}6.36 \\
6.23\end{array}$ & $\begin{array}{l}z^{5} \mathrm{D}_{0}^{\circ}-e{ }^{7} \mathrm{G}_{1} \\
b^{3} \mathrm{G}^{3}-v\end{array}$ & $\begin{array}{l}561 \\
427\end{array}$ & $\begin{array}{l}\text { Pred } \\
\text { Pred }\end{array}$ & $\mathrm{U}$ \\
\hline 3992. 634 & 1 & 25039. 04 & 9.01 & $b^{3} \mathrm{~F}_{3}-x{ }^{5} \mathrm{G}_{3}^{0}$ & 219 & Pred & BK, U \\
\hline $\begin{array}{l}\text { 3989. } 006 \\
3984.930 \\
3984.446 \\
3980.008 \\
3970.994\end{array}$ & $\begin{array}{l}1 N \\
1 \\
1 \\
1 \\
1\end{array}$ & $\begin{array}{l}25061.81 \\
25087.45 \\
25090.50 \\
25118.47 \\
25175.49\end{array}$ & $\begin{array}{l}\text { 1. } 93 \\
\text { 7. } 47 \\
\text { 0. } 46 \\
\text { 8. } 56 \\
\text { 5. } 54\end{array}$ & $\begin{array}{l}a{ }^{1} \mathrm{H}_{5}-53882_{4}^{\circ} \\
z^{5} \mathrm{D}_{1}^{\circ}-e{ }^{7} \mathrm{G}_{1} \\
b^{3} \mathrm{~F}_{3}-x{ }^{5} \mathrm{G}_{2}^{\circ} \\
b^{3} \mathrm{G}_{3}-495_{4}{ }^{\circ}{ }_{4}^{\circ} ? \\
y^{5} \mathrm{D}_{4}^{\circ}-g{ }^{5} \mathrm{G}_{5}\end{array}$ & $\begin{array}{r}561 \\
219 \\
1074\end{array}$ & $\begin{array}{l}\text { New } \\
\text { Pred } \\
\text { Pred } \\
\text { New } \\
\text { Pred }\end{array}$ & $\begin{array}{c}\mathrm{Z} \\
\mathrm{Z} \\
\mathrm{BK} \\
\mathrm{U} \\
\mathrm{U}\end{array}$ \\
\hline $\begin{array}{l}3963.438 \\
3962.635 \\
3953.512 \\
3951.638 \\
3948.458\end{array}$ & $\begin{array}{l}1 \\
0 N \\
2 \\
1 N \\
1\end{array}$ & $\begin{array}{l}25223.48 \\
25228.60 \\
25286.81 \\
25298.80 \\
25319.18\end{array}$ & $\begin{array}{l}\text { 3. } 58 \\
\text { 8. } 49 \\
6.93 \\
\text { 8. } 83 \\
9.06\end{array}$ & $\begin{array}{l}a{ }^{3} \mathrm{D}_{1}-t{ }^{5} \mathrm{D}_{2}^{\circ} \\
b^{3} \mathrm{D}_{3}-t{ }^{3} \mathrm{G}_{3}^{\circ} \\
a^{1} \mathrm{D}_{2}-10_{3}^{\circ} \\
b^{3} \mathrm{P}_{0}-v{ }^{5} \mathrm{~F}_{1}^{\circ} \\
z^{5} \mathrm{D}_{4}^{\circ}-e{ }^{5} \mathrm{G}_{3}^{\circ}\end{array}$ & $\begin{array}{l}654 \\
913 \\
770 \\
362 \\
560\end{array}$ & $\begin{array}{l}\text { Pred b } \\
\text { Pred } \\
\text { Pred b } \\
\text { New } \\
\text { Pred }\end{array}$ & $\begin{array}{c}\mathrm{U} \\
\mathrm{U} \\
\mathrm{U} \\
\mathrm{BK}\end{array}$ \\
\hline 3930.876 & $0 N$ & 25432. 42 & 2. 46 & $a^{3} \mathrm{H}_{4}-x{ }^{3} \mathrm{D}_{3}^{\circ} ?$ & & New & $\mathrm{U}$ \\
\hline 3922. 100 & $1 N$ & 25489.33 & $\begin{array}{l}9.51 \\
9.44\end{array}$ & $\begin{array}{l}z^{7} \mathrm{D}_{1}^{\circ}-e{ }^{5} \mathrm{D}_{1} \\
z^{5} \mathrm{D}_{0}^{\circ}-e{ }^{3} \mathrm{D}_{1}\end{array}$ & $\begin{array}{l}153 \\
564\end{array}$ & $\begin{array}{l}\text { Pred } \\
\text { Pred }\end{array}$ & $\mathrm{BK}, \quad \mathrm{U}$ \\
\hline $\begin{array}{l}\text { 3908. } 691 \\
3892.302 \\
3889.284\end{array}$ & $\begin{array}{l}1 N \\
1 \\
1 N\end{array}$ & $\begin{array}{l}25576.77 \\
25684.46 \\
25704.39\end{array}$ & $\begin{array}{l}\text { 6. } 84 \\
\text { 4. } 48 \\
\text { 4. } 46\end{array}$ & $\begin{array}{l}z^{7} \mathrm{D}_{3}^{\circ}-e{ }^{5} \mathrm{D}_{2} \\
a^{1} \mathrm{D}_{2}-54289_{3}^{\circ} \\
a^{3} \mathrm{G}_{5}-w^{5} \mathrm{G}_{5}^{\circ}\end{array}$ & $\begin{array}{l}153 \\
280\end{array}$ & $\begin{array}{l}\text { Pred } \\
\text { New } \\
\text { New }\end{array}$ & $\begin{array}{c}\mathrm{BK} \\
\mathrm{U} \\
\mathrm{U}\end{array}$ \\
\hline $\begin{array}{l}3847.226 \\
3847.077 \\
3842.901 \\
3819.497 \\
3816.908\end{array}$ & $\begin{array}{l}1 N \\
1 N \\
2 \\
2 N \\
1\end{array}$ & $\begin{array}{l}\text { 25985. } 38 \\
25986.39 \\
26014.63 \\
26174.03 \\
\text { 26191. } 78\end{array}$ & $\begin{array}{l}\text { 5. } 44 \\
\text { 6. } 28 \\
\text { 4. } 69 \\
\text { 4. } 02 \\
\text { 1. } 74\end{array}$ & 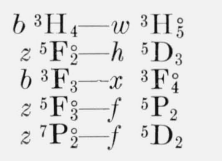 & $\begin{array}{l}607 \\
702 \\
222 \\
703 \\
387\end{array}$ & $\begin{array}{l}\text { New } \\
\text { New } \\
\text { Pred } \\
\text { Pred } \\
\text { Pred }\end{array}$ & $\begin{array}{c}\text { BK, U } \\
\text { BK, Z } \\
\text { Z } \\
\text { U }\end{array}$ \\
\hline $\begin{array}{l}3814.785 \\
3811.808 \\
3803.220 \\
3801.337 \\
3789.808\end{array}$ & $\begin{array}{l}1 \\
0 \\
1 N \\
1 \\
1 N\end{array}$ & $\begin{array}{l}\text { 26206. } 36 \\
26226.83 \\
26286.05 \\
26299.07 \\
26379.07\end{array}$ & $\begin{array}{l}\text { 6. } 39 \\
\text { 6. } 88 \\
\text { 5. } 91 \\
\text { 8. } 89 \\
\text { 9. } 04\end{array}$ & 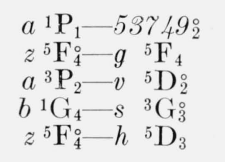 & $\begin{array}{l}701 \\
122 \\
948 \\
702\end{array}$ & $\begin{array}{l}\text { New } \\
\text { Pred } \\
\text { Pred } \\
\text { New } \\
\text { Pred }\end{array}$ & $\begin{array}{c}\mathrm{Z} \\
\mathrm{BK}, \mathrm{U} \\
\mathrm{U} \\
\mathrm{Z} \\
\mathrm{Z}\end{array}$ \\
\hline $\begin{array}{l}\text { 3789. } 292 \\
3784.127 \\
3771.473 \\
\text { 3769. } 766 \\
3759.597\end{array}$ & $\begin{array}{l}0 \\
3 n \\
2 \\
0 \\
1 n\end{array}$ & $\begin{array}{l}\text { 26382. } 66 \\
26418.67 \\
26507.31 \\
26519.31 \\
26591.04\end{array}$ & $\begin{array}{l}\text { 2. } 57 \\
\text { 8. } 86 \\
\text { 7. } 13 \\
\text { 9. } 15 \\
\text { 1. } 16\end{array}$ & $\begin{array}{l}a^{3} \mathrm{P}_{2}-v{ }^{5} \mathrm{D}_{1}^{\circ} \\
b^{3} \mathrm{D}_{3}-w^{1} \mathrm{~F}_{3}^{\circ} \\
b^{3} \mathrm{H}_{6}-w^{3} \mathrm{H}_{5}^{\circ} \\
z^{5} \mathbf{F}_{5}^{\circ}-g{ }^{5} \mathbf{F}_{4} \\
z^{5} \mathbf{F}_{1}^{\circ}-g{ }^{5} \mathrm{~F}_{2}\end{array}$ & $\begin{array}{l}122 \\
917 \\
607 \\
701 \\
701\end{array}$ & $\begin{array}{l}\text { New } \\
\text { New } \\
\text { Pred } \\
\text { New } \\
\text { New }\end{array}$ & $\begin{array}{c}\mathrm{U} \\
\mathrm{BK}, \mathrm{Z} \\
\mathrm{U}\end{array}$ \\
\hline $\begin{array}{l}\text { 3751. } 087 \\
3743.778 \\
3742.151 \\
3741.486 \\
3739.527\end{array}$ & $\begin{array}{l}2 \\
2 \\
2 n \\
1 n \\
3\end{array}$ & $\begin{array}{l}26651.36 \\
26703.39 \\
26715.00 \\
26719.75 \\
26733.75\end{array}$ & $\begin{array}{l}\text { 1. } 42 \\
\text { 3. } 40 \\
\text { 5. } 08 \\
\text { 9. } 80 \\
\text { 3. } 80\end{array}$ & $\begin{array}{c}a^{5} \mathrm{P}_{2}-w{ }^{5} \mathrm{~F}_{1} \\
a^{3} \mathrm{G}_{4}-y \\
{ }^{1} \mathrm{G}_{4}^{\circ} \\
z^{3} \mathrm{~F}_{3}^{\circ}-g{ }^{5} \mathrm{G}_{4} \\
z^{5} \mathrm{~F}_{1}-g{ }^{5} \mathrm{~F}_{1} \\
a^{3} \mathrm{D}_{3}-53358_{3}^{\circ}\end{array}$ & $\begin{array}{r}74 \\
290 \\
978 \\
701\end{array}$ & $\begin{array}{l}\text { Pred d } \\
\text { SS } \\
\text { Pred } \\
\text { New } \\
\text { New }\end{array}$ & $\begin{array}{c}\mathrm{Z} \\
\mathrm{Z}, \mathrm{ZZ} \\
\underset{\mathrm{U}}{\mathrm{BK}}, \mathrm{Z}\end{array}$ \\
\hline $\begin{array}{l}\text { 3707. } 578 \\
3707.458 \\
3707.335 \\
3699.004 \\
3698.148\end{array}$ & $\begin{array}{l}1 n \\
2 \\
1 \\
0 \\
1 n\end{array}$ & $\begin{array}{l}26964.11 \\
26964.99 \\
26965.88 \\
27026.61 \\
27032.87\end{array}$ & $\begin{array}{l}\text { 4. } 23 \\
\text { 4. } 96 \\
\text { 5. } 85 \\
\text { 6. } 50 \\
\text { 2. } 84\end{array}$ & 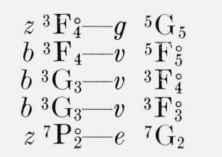 & $\begin{array}{l}978 \\
229 \\
437 \\
437 \\
390\end{array}$ & $\begin{array}{l}\text { New } \\
\text { New } \\
\text { New } \\
\text { New } \\
\text { New }\end{array}$ & $\begin{array}{l}Z \\
Z \\
U\end{array}$ \\
\hline
\end{tabular}


Table 3. Classified faint lines of Fe I-Continued

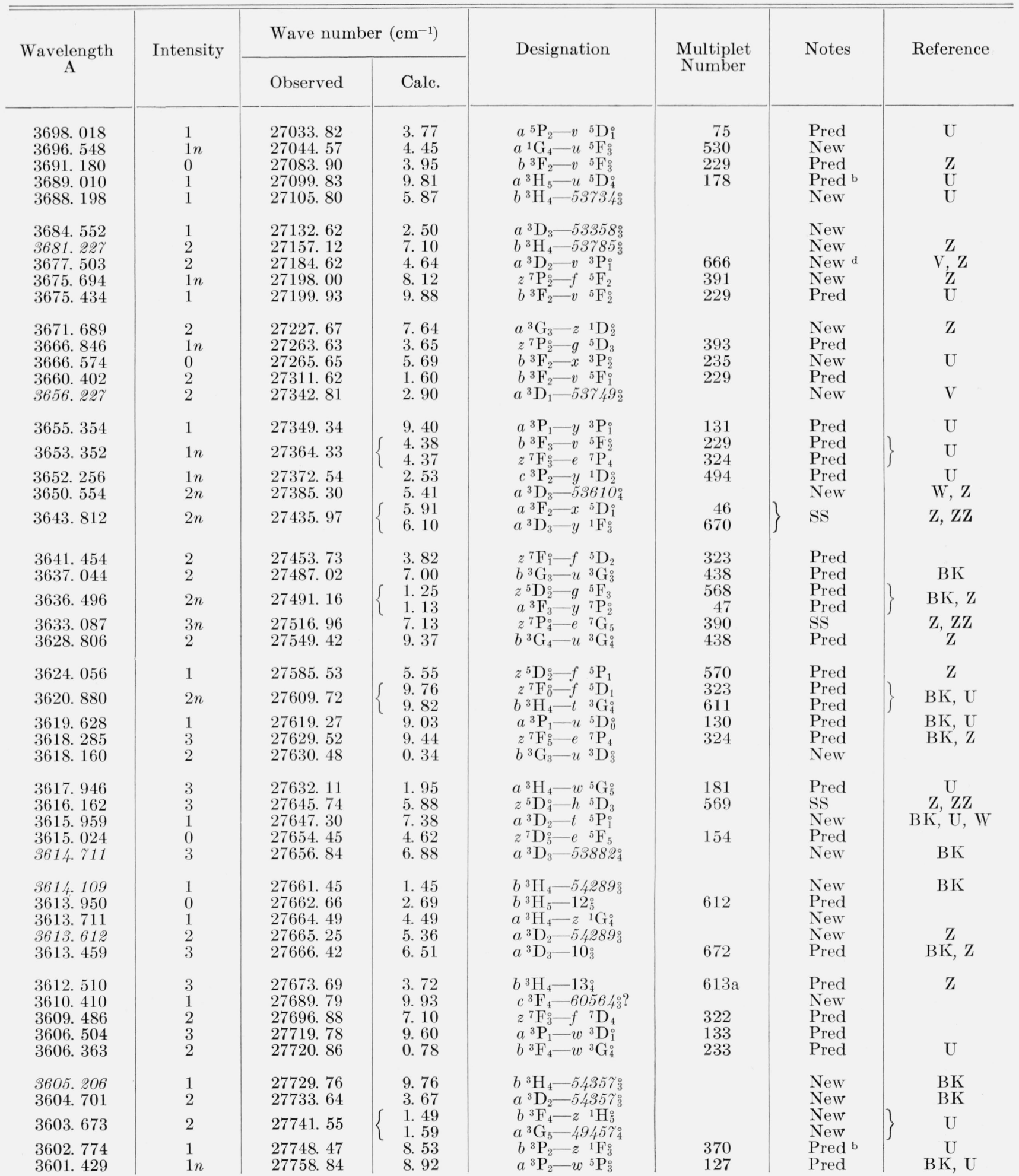


TABLE 3. Classified faint lines of Fe I-Continued

\begin{tabular}{|c|c|c|c|c|c|c|c|}
\hline \multirow{2}{*}{$\begin{array}{c}\text { Wavelength } \\
\text { A }\end{array}$} & \multirow{2}{*}{ Intensity } & \multicolumn{2}{|c|}{ Wave number $\left(\mathrm{cm}^{-1}\right)$} & \multirow{2}{*}{ Designation - } & \multirow{2}{*}{$\begin{array}{l}\text { Multiplet } \\
\text { Number }\end{array}$} & \multirow{2}{*}{ Notes } & \multirow{2}{*}{ Reference } \\
\hline & & Observed & Calc. & & & & \\
\hline $\begin{array}{l}\text { 3593. } 764 \\
3591.998 \\
3589.586 \\
3588.516 \\
358 \% .752\end{array}$ & $\begin{array}{l}0 n \\
0 \\
2 \\
3 \\
3\end{array}$ & $\begin{array}{l}27818.04 \\
27831.72 \\
27850.42 \\
27858.72 \\
27864.65\end{array}$ & $\begin{array}{l}\text { 7. } 82 \\
\text { 1. } 73 \\
\text { 0. } 29 \\
\text { 8. } 69 \\
\text { 4. } 62\end{array}$ & $\begin{array}{cc}a^{3} \mathrm{H}_{4}-v & { }^{5} \mathrm{~F}_{5}^{\circ} \\
a^{3} \mathrm{H}_{5}-z & { }^{1} \mathrm{G}_{4}^{\circ} \\
b^{3} \mathrm{G}_{4}-u & { }^{3} \mathrm{D}_{3}^{\circ} \\
z^{7} \mathrm{P}_{4}^{\circ}-e & { }^{7} \mathrm{~S}_{3} \\
a^{3} \mathrm{D}_{1}-t & { }^{5} \mathrm{P}_{1}^{\circ}\end{array}$ & $\begin{array}{l}182 \\
394\end{array}$ & $\begin{array}{l}\text { Pred } \\
\text { New } \\
\text { New } \\
\text { Pred } \\
\text { New }\end{array}$ & $\underset{J}{\mathrm{BK}, \mathrm{U}}$ \\
\hline $\begin{array}{l}3586.740 \\
\text { 3582. } 324 \\
3579.829 \\
\text { 3575. } 754 \\
\text { 3574. } 364\end{array}$ & $\begin{array}{l}3 n \\
2 n \\
1 \\
1 \\
0\end{array}$ & $\begin{array}{l}27872.52 \\
27906.87 \\
27926.32 \\
27958.15 \\
27969.02\end{array}$ & $\begin{array}{l}2.51 \\
6.77 \\
6.38 \\
8.16 \\
9.03\end{array}$ & $\begin{array}{l}z^{7} \mathrm{~F}_{6}^{\circ}-e{ }^{5} \mathrm{G}_{6} \\
z^{5} \mathrm{D}_{1}^{\circ}-g{ }^{5} \mathrm{~F}_{1} \\
z^{5} \mathrm{D}_{3}^{\circ}-e{ }^{3} \mathrm{G}_{4} \\
b^{3} \mathrm{G}_{3}-u{ }^{3} \mathrm{D}_{2}^{\circ} \\
a^{3} \mathrm{H}_{5}-w^{5} \mathrm{G}_{4}^{\circ}\end{array}$ & $\begin{array}{l}325 \\
568 \\
573 \\
\\
181\end{array}$ & $\begin{array}{l}\text { SS } \\
\text { Pred } \\
\text { Pred } \\
\text { New } \\
\text { Pred b }\end{array}$ & $\begin{array}{l}\mathrm{Z}, \mathrm{ZZ} \\
\mathrm{BK}, \mathrm{U} \\
\mathrm{BK}, \mathrm{Z}\end{array}$ \\
\hline $\begin{array}{l}3574.256 \\
3567.748 \\
3564.533 \\
3563.618 \\
3562.269\end{array}$ & $\begin{array}{l}1 \\
1 \\
3 \\
1 \\
1 n\end{array}$ & $\begin{array}{l}27969.86 \\
28020.88 \\
28046.16 \\
28053.36 \\
28063.98\end{array}$ & $\begin{array}{l}9.94 \\
0.99 \\
6.34 \\
\text { 3. } 48 \\
\text { 4. } 06\end{array}$ & $\begin{array}{cc}z^{5} \mathrm{D}_{1}^{\circ}-f & { }^{3} \mathrm{D}_{1} \\
z^{5} \mathrm{D}_{3}^{\circ}-f & { }^{5} \mathrm{G}_{3} \\
a^{3} \mathrm{H}_{4}-x & { }^{3} \mathrm{G}_{3}^{\circ} \\
z^{7} \mathrm{~F}_{6}^{\circ}-e & { }^{5} \mathrm{G}_{5} \\
a^{3} \mathrm{D}_{3}-5 & 4289_{3}^{\circ}\end{array}$ & $\begin{array}{l}574 \\
571 \\
183 \\
325\end{array}$ & $\begin{array}{l}\text { New } \\
\text { New } \\
\text { Pred } \\
\text { Pred } \\
\text { New }\end{array}$ & $\underset{\mathrm{U}}{\mathrm{BK}}, \mathrm{Z}$ \\
\hline $\begin{array}{l}3560.076 \\
3551.114 \\
3530.976 \\
3528.942 \\
3528.316\end{array}$ & $\begin{array}{l}1 \\
1 \\
1 \\
1 \\
0\end{array}$ & $\begin{array}{l}\text { 28081. } 27 \\
28152.14 \\
28312.69 \\
28329.01 \\
28334.03\end{array}$ & $\begin{array}{l}\text { 1. } 37 \\
\text { 2. } 18 \\
\text { 2. } 76 \\
\text { 9. } 06 \\
\text { 4. } 07\end{array}$ & 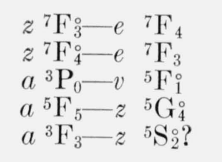 & $\begin{array}{r}321 \\
321 \\
138 \\
23\end{array}$ & $\begin{array}{l}\text { Pred } \\
\text { Pred } \\
\text { New } \\
\text { Pred b } \\
\text { New }\end{array}$ & $\underset{\mathrm{U}}{\mathrm{BK}, \mathrm{Z}}$ \\
\hline $\begin{array}{l}3528.233 \\
3515.404 \\
3509.736 \\
3507.139 \\
\text { 3502. } 853\end{array}$ & $\begin{array}{l}1 \\
1 \\
3 \\
3 \\
1\end{array}$ & $\begin{array}{l}\text { 28334. } 70 \\
28438.10 \\
28484.02 \\
28505.12 \\
28539.99\end{array}$ & $\begin{array}{l}\text { 4. } 69 \\
\text { 8. } 08 \\
\text { 4. } 14 \\
\text { 5. } 13 \\
0.01\end{array}$ & $\begin{array}{cc}a^{3} \mathrm{H}_{4}-v & { }^{5} \mathrm{~F}_{3}^{\circ} \\
b^{3} \mathrm{~F}_{2}-z & { }^{1} \mathrm{D}_{2}^{\circ} \\
z^{7} \mathrm{~F}_{0}^{\circ}-f & { }^{5} \mathrm{~F}_{1} \\
z^{5} \mathrm{P}_{2}^{\circ}-i & { }^{5} \mathrm{D}_{2} \\
z^{5} \mathrm{D}_{2}^{\circ}-e & { }^{3} \mathrm{P}_{2}\end{array}$ & $\begin{array}{l}182 \\
243 \\
327 \\
835 \\
577\end{array}$ & $\begin{array}{l}\text { Pred } \\
\text { Pred } \\
\text { Pred } \\
\text { Pred } \\
\text { Pred b }\end{array}$ & $\begin{array}{l}\mathrm{U} \\
\mathrm{U} \\
\mathrm{U} \\
\mathrm{Z}\end{array}$ \\
\hline $\begin{array}{l}3500.164 \\
3498.755 \\
3487.138 \\
3481.292 \\
34 \text { \%. } 303\end{array}$ & $\begin{array}{l}2 \\
2 \\
0 \\
1 \\
2\end{array}$ & $\begin{array}{l}\text { 28561. } 92 \\
28573.42 \\
28668.61 \\
28716.75 \\
28782.80\end{array}$ & $\begin{array}{l}\text { 2. } 03 \\
\text { 3. } 47 \\
\text { 8. } 49 \\
\text { 6. } 75 \\
\text { 2. } 84\end{array}$ & $\begin{array}{l}z^{7} \mathrm{~F}_{2}^{\circ}-f{ }^{5} \mathrm{~F}_{1} \\
z^{7} \mathrm{~F}_{4}^{\circ}-e{ }^{7} \mathrm{~S}_{3} \\
c^{3} \mathrm{~F}_{3}-62081_{2}^{\circ} ? \\
c^{3} \mathrm{P}_{0}-v{ }^{3} \mathrm{P}_{1} \\
a^{1} \mathrm{G}_{4}-53358_{3}^{\circ}\end{array}$ & $\begin{array}{l}327 \\
330 \\
\\
499\end{array}$ & $\begin{array}{l}\text { New } \\
\text { New } \\
\text { New } \\
\text { New } \\
\text { New }\end{array}$ & $\begin{array}{c}\mathrm{U} \\
\mathrm{W}, \mathrm{Z} \\
\mathrm{V}\end{array}$ \\
\hline $\begin{array}{l}3473.015 \\
3469.278 \\
3467.686 \\
3456.374 \\
3448.190\end{array}$ & $\begin{array}{l}0 n \\
0 \\
1 \\
1 n \\
1\end{array}$ & $\begin{array}{l}\text { 28785. } 18 \\
28816.19 \\
\text { 28829. } 42 \\
28923.77 \\
28992.41\end{array}$ & $\begin{array}{l}\text { 5. } 27 \\
\text { 6. } 22 \\
\text { 9. } 43 \\
\text { 3. } 76 \\
\text { 2. } 43\end{array}$ & $\begin{array}{c}z^{5} \mathrm{D}_{2}^{\circ}-f{ }^{3} \mathrm{~F}_{3} \\
b^{3} \mathrm{~F}_{4}-495^{4} \gamma_{4}^{\circ} ? \\
b^{3} \mathrm{G}_{5}-w^{3} \mathrm{H}_{5}^{\circ} \\
b^{3} \mathrm{P}_{2}-x \\
a^{3} \mathrm{D}_{6} \mathrm{D}_{2}^{\circ} \\
{ }^{3} \mathrm{H}_{6} \mathrm{H}_{5}^{\circ}\end{array}$ & $\begin{array}{l}576 \\
442 \\
375 \\
186\end{array}$ & $\begin{array}{l}\text { Pred } \\
\text { New } \\
\text { New } \\
\text { New } \\
\text { Pred }\end{array}$ & BK, U \\
\hline $\begin{array}{l}3434.960 \\
3429.808 \\
3418.905 \\
3414.564 \\
3410.581\end{array}$ & $\begin{array}{l}1 n \\
1 \\
1 \\
1 \\
0 n\end{array}$ & $\begin{array}{l}\text { 29104. } 08 \\
\text { 29147. } 79 \\
\text { 29240. } 74 \\
29277.92 \\
29312.11\end{array}$ & $\begin{array}{l}\text { 4. } 15 \\
\text { 7. } 75 \\
\text { 7. } 85 \\
\text { 0. } 62 \\
\text { 7. } 92 \\
\text { 2. } 35\end{array}$ & 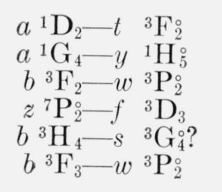 & $\begin{array}{l}776 \\
540 \\
244\end{array}$ & $\begin{array}{l}\text { Pred } \\
\text { Pred } \\
\text { Pred } \\
\text { New } \\
\text { New } \\
\text { Pred }\end{array}$ & $\underset{\mathrm{U}}{\mathrm{W}, \mathrm{Z}}$ \\
\hline $\begin{array}{l}3409.605 \\
3401.007 \\
3400.662 \\
3395.080 \\
3393.623\end{array}$ & $\begin{array}{l}1 \\
1 \\
1 \\
1 \\
2\end{array}$ & $\begin{array}{l}29320.50 \\
29394.62 \\
29397.60 \\
29445.94 \\
29458.58\end{array}$ & $\begin{array}{l}\text { 0. } 66 \\
\text { 4. } 71 \\
\text { 7. } 71 \\
\text { 5. } 94 \\
\text { 8. } 60\end{array}$ & $\begin{array}{c}a^{3} \mathrm{H}_{4}-w^{3} \mathrm{~F}_{4}^{\circ} \\
b^{3} \mathrm{G}_{3}-537344^{\circ} \\
c^{3} \mathrm{P}_{2}-537343 \\
b^{3} \mathrm{G}_{3}-53785_{3}^{\circ} \\
b^{3} \mathrm{P}_{2}-u{ }^{3} \mathrm{D}_{2}^{\circ}\end{array}$ & 188 & $\begin{array}{l}\text { New } \\
\text { New } \\
\text { New } \\
\text { New } \\
(a, d)\end{array}$ & $\underset{\mathrm{V}}{\mathrm{UK}} \underset{\mathrm{U}}{\mathrm{U}} \mathrm{U}$ \\
\hline $\begin{array}{l}3393.590 \\
3384.765 \\
3381.990 \\
3381.498 \\
3375.724\end{array}$ & $\begin{array}{l}1 \\
1 \\
1 n \\
1 n \\
1\end{array}$ & $\begin{array}{l}29458.86 \\
29535.67 \\
29559.90 \\
29564.20 \\
29614.77\end{array}$ & $\begin{array}{l}\text { 8. } 87 \\
\text { 5. } 39 \\
\text { 9. } 89 \\
\text { 4. } 19 \\
\text { 4. } 66\end{array}$ & 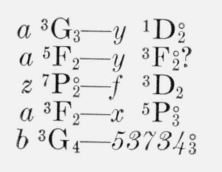 & $\begin{array}{r}305 \\
25 \\
49\end{array}$ & $\begin{array}{l}(a, d) \\
\text { Pred b } \\
\text { New } \\
\text { New } \\
\text { New }\end{array}$ & $U, V$ \\
\hline $\begin{array}{l}3374.176 \\
3370.254 \\
3369.146 \\
3364.402 \\
\text { 3358. } 911\end{array}$ & $\begin{array}{l}2 \\
0 n \\
2 \\
1 \\
2\end{array}$ & $\begin{array}{l}29628.36 \\
29662.83 \\
29672.59 \\
29714.43 \\
29763.00\end{array}$ & $\begin{array}{l}\text { 8. } 22 \\
\text { 2. } 77 \\
\text { 2. } 64 \\
\text { 4. } 40 \\
\text { 3. } 06\end{array}$ & $\begin{array}{l}a{ }^{5} \mathrm{P}_{1}-y{ }^{3} \mathrm{~S}_{1} \\
a^{1} \mathrm{G}_{4}-t \\
a^{3} \mathrm{G}_{4}^{\circ} \mathrm{H}_{4}-v \\
{ }^{3} \mathrm{G}_{5}^{\circ} \\
a^{1} \mathrm{G}_{4}-54289_{3}^{\circ} \\
b^{3} \mathrm{G}_{4}-53882_{4}^{\circ}\end{array}$ & $\begin{array}{l}89 \\
542 \mathrm{a} \\
191\end{array}$ & $\begin{array}{l}(a) \\
\text { New } \\
\text { Pred } \\
\text { New } \\
\text { New }\end{array}$ & $\begin{array}{l}\mathrm{Z} \\
\mathrm{Z} \\
\mathrm{V}\end{array}$ \\
\hline
\end{tabular}


TABLE 3. Classified faint lines of Fe $\mathrm{I}$-Continued

\begin{tabular}{|c|c|c|c|c|c|c|c|}
\hline \multirow{2}{*}{$\begin{array}{c}\text { Wavelength } \\
\text { A }\end{array}$} & \multirow{2}{*}{ Intensity } & \multicolumn{2}{|c|}{ Wave number $\left(\mathrm{cm}^{-1}\right)$} & \multirow{2}{*}{ Designation } & \multirow{2}{*}{$\begin{array}{l}\text { Multiplet } \\
\text { Number }\end{array}$} & \multirow{2}{*}{ Notes } & \multirow{2}{*}{ Reference } \\
\hline & & Observed & Calc. & & & & \\
\hline $\begin{array}{l}3357.823 \\
3356.695 \\
3344.078 \\
3337.915 \\
3330.316\end{array}$ & $\begin{array}{l}0 \\
3 \\
0 \\
1 \\
1 n\end{array}$ & $\begin{array}{l}\text { 29772. } 64 \\
29782.65 \\
29895.01 \\
29950.21 \\
30018.55\end{array}$ & $\begin{array}{l}\text { 2. } 69 \\
\text { 2. } 71 \\
\text { 4. } 93 \\
\text { 0. } 29 \\
\text { 8. } 60\end{array}$ & $\begin{array}{l}b^{3} \mathrm{G}_{4}-10_{3}^{\circ} \\
a^{1} \mathrm{G}_{4}-5435 \gamma_{3}^{\circ} \\
b^{3} \mathrm{G}_{4}-12_{5}^{\circ} \\
b^{3} \mathrm{G}_{3}-54289_{3}^{\circ} \\
b^{3} \mathrm{G}_{3}-54357_{3}^{\circ}\end{array}$ & $\begin{array}{l}448 \\
450\end{array}$ & $\begin{array}{l}\text { Pred b } \\
\text { New } \\
\text { Pred } \\
\text { New } \\
\text { New }\end{array}$ & $\begin{array}{l}\mathrm{U} \\
\mathrm{V} \\
\mathrm{U} \\
\mathrm{U} \\
\mathrm{V}\end{array}$ \\
\hline $\begin{array}{l}3330.206 \\
3329.970 \\
3316.558 \\
3315.164 \\
3313.555\end{array}$ & $\begin{array}{l}1 \\
1 n \\
1 \\
1 \\
0 n\end{array}$ & $\begin{array}{l}30019.54 \\
30021.67 \\
30143.07 \\
30155.74 \\
30170.38\end{array}$ & $\begin{array}{l}\text { 9. } 48 \\
\text { 1. } 60 \\
\text { 3. } 08 \\
\text { 5. } 69 \\
\text { 0. } 24\end{array}$ & $\begin{array}{l}b^{3} \mathrm{P}_{2}-x{ }^{3} \mathrm{~S}_{1}^{\circ} \\
c^{3} \mathrm{P}_{2}-5435 \gamma_{3}^{\circ} \\
a^{5} \mathrm{P}_{3}-w^{5} \mathrm{G}_{3}^{\circ} ? \\
b^{3} \mathrm{H}_{4}-u{ }^{3} \mathrm{~F}_{3}^{\circ} \\
b^{3} \mathrm{G}_{4}-54289_{3}^{\circ}\end{array}$ & $\begin{array}{r}378 \\
86 \\
618\end{array}$ & $\begin{array}{l}\text { New } \\
\text { New } \\
\text { New } \\
\text { Pred } \\
\text { New }\end{array}$ & $\begin{array}{l}\mathrm{U} \\
\mathrm{V} \\
\mathrm{U}\end{array}$ \\
\hline $\begin{array}{l}3304.346 \\
3298.537 \\
3291.410 \\
3281.824 \\
3276.978\end{array}$ & $\begin{array}{l}1 n \\
1 \\
0 \\
1 \\
0\end{array}$ & $\begin{array}{l}\text { 30254. } 46 \\
\text { 30307. } 74 \\
\text { 30373. } 37 \\
\text { 30462. } 08 \\
\text { 30507. } 13\end{array}$ & $\begin{array}{l}\text { 4. } 37 \\
\text { 7. } 80 \\
\text { 3. } 10 \\
\text { 2. } 04 \\
\text { 7. } 14\end{array}$ & $\begin{array}{cc}z^{5} \mathrm{~F}_{2}^{\circ}-i & { }^{5} \mathrm{D}_{3} \\
z^{5} \mathrm{~F}_{1}-i & { }^{5} \mathrm{D}_{2} \\
b^{1} \mathrm{G}_{4}-r & { }^{3} \mathrm{G}_{4} \\
a^{3} \mathrm{~F}_{3}-y & { }^{5} \mathrm{G}_{4} \\
z^{7} \mathrm{~F}_{5}^{\circ}-e & { }^{5} \mathrm{H}_{6}\end{array}$ & $\begin{array}{r}710 \\
710 \\
954 \\
50 \\
51\end{array}$ & $\begin{array}{l}\text { Pred } \\
\text { New } \\
\text { Pred } \\
\text { Pred b } \\
\text { New }\end{array}$ & $\begin{array}{l}\mathrm{U} \\
\mathrm{U} \\
\mathrm{U} \\
\mathrm{U}\end{array}$ \\
\hline $\begin{array}{l}3272.596 \\
3269.416 \\
3263.683 \\
3263.487 \\
3261.801\end{array}$ & $\begin{array}{l}2 \\
2 \\
0 \\
0 \\
0\end{array}$ & $\begin{array}{l}\text { 30547. } 98 \\
30577.69 \\
30631.40 \\
30633.24 \\
30649.07\end{array}$ & $\begin{array}{l}\text { 7. } 99 \\
\text { 7. } 69 \\
\text { 1. } 31 \\
\text { 3. } 62 \\
\text { 9. } 09\end{array}$ & 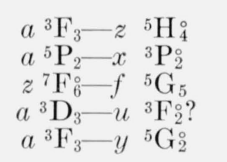 & $\begin{array}{r}95 \\
680 \\
50\end{array}$ & $\begin{array}{l}\text { Pred } \\
\text { Pred } \\
\text { New } \\
\text { Pred } \\
\text { New }\end{array}$ & $\begin{array}{l}\underset{\mathrm{U}}{\mathrm{Z}}, \mathrm{U} \\
\mathrm{BK}, \mathrm{U}\end{array}$ \\
\hline $\begin{array}{l}3258.627 \\
3249.504 \\
3241.502 \\
3240.145 \\
3238.313\end{array}$ & $\begin{array}{l}1 \\
1 \\
0 \\
0 n \\
0\end{array}$ & $\begin{array}{l}\text { 30678. } 92 \\
\text { 30765. } 05 \\
\text { 30841. } 00 \\
\text { 30853. } 91 \\
\text { 30871. } 37\end{array}$ & $\begin{array}{l}\text { 9. } 02 \\
\text { 5. } 03 \\
\text { 1. } 04 \\
\text { 4. } 26 \\
\text { 1. } 37\end{array}$ & $\begin{array}{cc}z^{7} \mathrm{D}_{1}^{\circ}-f & { }^{5} \mathrm{D}_{2} \\
a^{3} \mathrm{~F}_{3}-z & { }^{5} \mathrm{H}_{3}^{\circ} \\
a^{5} \mathrm{~F}_{1}-y & { }^{3} \mathrm{D}_{1}^{\circ} \\
z^{7} \mathrm{D}_{3}^{\circ}-e & { }^{7} \mathrm{P}_{3} \\
a^{1}{ }^{1} \mathrm{G}_{4}-v & { }^{3} \mathrm{H}_{4}^{\circ}\end{array}$ & $\begin{array}{r}157 \\
51 \\
27 \\
158 \\
545\end{array}$ & $\begin{array}{l}\text { Pred } \\
\text { New } \\
\text { (a) } \\
\text { Pred } \\
\text { Pred }\end{array}$ & $\mathrm{BK}, \underset{\underset{\mathrm{U}}{\mathrm{U}}}{\mathrm{U}}, \mathrm{W}$ \\
\hline $\begin{array}{l}\text { 3235. } 833 \\
3235.312 \\
3232.155 \\
3231.356 \\
3230.085\end{array}$ & $\begin{array}{l}1 \\
1 \\
1 \\
1 \\
1\end{array}$ & $\begin{array}{l}\text { 30895. } 03 \\
30900.00 \\
30930.18 \\
30937.83 \\
30950.00\end{array}$ & $\begin{array}{l}\text { 5. } 15 \\
9.89 \\
\text { 0. } 12 \\
\text { 7. } 71 \\
\text { 5. } 00\end{array}$ & $\begin{array}{c}b^{3} \mathrm{P}_{2}-537344^{\circ} \\
a^{3} \mathrm{G}_{4}-y{ }^{3} \mathrm{I}_{5}^{\circ} \\
b^{3} \mathrm{~F}_{2}-u^{3} \mathrm{D}_{3}^{\circ} \\
b^{3} \mathrm{H}_{4}-57565_{3}^{\circ} ? \\
a^{5} \mathrm{~F}_{3}-y{ }^{3} \mathrm{D}_{2}^{\circ}\end{array}$ & $\begin{array}{r}309 \\
258 \\
\\
27\end{array}$ & $\begin{array}{l}\text { New } \\
\text { Pred } \\
\text { Pred b } \\
\text { New } \\
\text { Pred }\end{array}$ & $\begin{array}{c}\mathrm{V} \\
\mathrm{U} \\
\mathrm{U} \\
\mathrm{U} \\
\mathrm{BK}\end{array}$ \\
\hline $\begin{array}{l}\text { 3229. } 595 \\
\text { 3226. } 012 \\
3223.480 \\
3223.080 \\
3219.187\end{array}$ & $\begin{array}{l}2 n \\
2 \\
1 n \\
0 \\
1 n\end{array}$ & $\begin{array}{l}\text { 30954. } 70 \\
\text { 30989. } 08 \\
\text { 31013. } 42 \\
\text { 31017. } 27 \\
\text { 31054. } 78\end{array}$ & $\begin{array}{l}\text { 5. } 02 \\
\text { 9. } 09 \\
\text { 3. } 42 \\
\text { 7. } 33 \\
\text { 4. } 96\end{array}$ & $\begin{array}{l}z^{7} \mathrm{~F}_{5}^{\circ}-g{ }^{7} \mathrm{D}_{5} ? \\
b^{3} \mathrm{~F}_{4}-6_{5}^{\circ} \\
b^{3} \mathrm{H}_{4}-t{ }^{3} \mathrm{~F}_{3}^{\circ} \\
a^{3} \mathrm{D}_{2}-t{ }^{3} \mathrm{~F}_{3}^{\circ} \\
a^{3} \mathrm{P}_{2}-w^{3} \mathrm{~F}_{2}^{\circ} ?\end{array}$ & $\begin{array}{l}333 \\
\\
682 \\
141\end{array}$ & $\begin{array}{l}\text { New } \\
\text { New } \\
\text { New c } \\
\text { Pred } \\
\text { New c }\end{array}$ & $\begin{array}{c}\mathrm{V} \\
\mathrm{BK}, \mathrm{Z} \\
\mathrm{Z} \\
\mathrm{U}\end{array}$ \\
\hline $\begin{array}{l}\text { 3205. } 782 \\
3204.306 \\
\text { 3199. } 920 \\
\text { 3195. } 968 \\
\text { 3193. } 726\end{array}$ & $\begin{array}{l}1 \\
1 \\
1 n \\
1 \\
0\end{array}$ & $\begin{array}{l}31184.63 \\
31198.99 \\
31241.75 \\
31280.38 \\
31302.34\end{array}$ & $\begin{array}{l}\text { 4. } 66 \\
\text { 9. } 00 \\
\text { 1. } 65 \\
\text { 0. } 49 \\
\text { 2. } 27\end{array}$ & 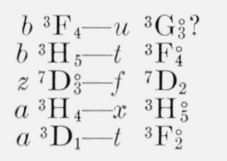 & $\begin{array}{l}252 \\
156 \\
192 \mathrm{a} \\
682\end{array}$ & $\begin{array}{l}\text { New } \\
\text { New } \\
\text { New } \\
\text { New } \\
\text { Pred }\end{array}$ & $\begin{array}{l}\mathrm{U} \\
\mathrm{U} \\
\mathrm{U} \\
\mathrm{U}\end{array}$ \\
\hline $\begin{array}{l}\text { 3188. } 026 \\
\text { 3187. } 171 \\
\text { 3186. } 814 \\
3184.112 \\
3176.278\end{array}$ & $\begin{array}{l}2 \\
1 n \\
1 \\
1 \\
1 n\end{array}$ & $\begin{array}{l}\text { 31358. } 31 \\
31366.72 \\
31370.23 \\
31396.85 \\
31474.28\end{array}$ & $\begin{array}{l}\text { 8. } 36 \\
\text { 6. } 87 \\
\text { 0. } 25 \\
\text { 6. } 94 \\
\text { 4. } 26\end{array}$ & 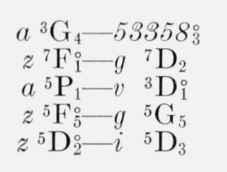 & $\begin{array}{l}333 \\
100 \\
711 \\
578\end{array}$ & $\begin{array}{l}\text { New } \\
\text { Pred } \\
\text { Pred b } \\
\text { New } \\
\text { New }\end{array}$ & $\begin{array}{c}\mathrm{Z} \\
\mathrm{BK}, \mathrm{Z} \\
\mathrm{U} \\
\mathrm{Z} \\
\mathrm{U}\end{array}$ \\
\hline $\begin{array}{l}3175.318 \\
3172.292 \\
3167.792 \\
3166.982 \\
3166.259\end{array}$ & $\begin{array}{l}1 \\
1 \\
1 \\
1 \\
2 n\end{array}$ & $\begin{array}{l}31483.80 \\
31513.83 \\
31558.60 \\
31566.67 \\
31573.88\end{array}$ & $\begin{array}{l}\text { 4. } 05 \\
\text { 3. } 82 \\
\text { 8. } 73 \\
\text { 6. } 76 \\
\text { 4. } 05\end{array}$ & $\begin{array}{l}a^{3} \mathrm{G}_{3}-53734_{3}^{\circ} ? \\
a^{3} \mathrm{G}_{3}-x{ }^{1} \mathrm{~F}_{3}^{\circ} \\
a^{5} \mathrm{P}_{3}-w^{3} \mathrm{~F}_{4}^{\circ} \\
b^{3} \mathrm{G}_{3}-s{ }^{3} \mathrm{G}_{4}^{\circ} \\
z^{7} \mathrm{D}_{3}^{\circ}-e{ }^{7} \mathrm{~F}_{2}\end{array}$ & $\begin{array}{r}312 \\
99 \\
455 \\
155\end{array}$ & $\begin{array}{l}\text { New } \\
\text { Pred } \\
\text { Pred b } \\
\text { Pred b } \\
\text { Pred }\end{array}$ & $\begin{array}{l}\mathrm{U} \\
\mathrm{U} \\
\mathrm{U} \\
\mathrm{Z}\end{array}$ \\
\hline $\begin{array}{l}\text { 3161. } 558 \\
3159.437 \\
3159.248 \\
3155.134 \\
3154.106\end{array}$ & $\begin{array}{l}1 \\
1 \\
1 \\
1 n \\
1\end{array}$ & $\begin{array}{l}31620.82 \\
31642.05 \\
31643.94 \\
31685.20 \\
31695.53\end{array}$ & $\begin{array}{l}0.90 \\
\text { 2. } 08 \\
\text { 3. } 91 \\
\text { 5. } 40 \\
\text { 5. } 56\end{array}$ & $\begin{array}{l}a^{3} \mathrm{H}_{4}-4_{4}^{\circ} \\
a^{3} \mathrm{G}_{3}-10_{3}^{\circ} \\
b^{3} \mathrm{~F}_{2}-t \\
{ }^{3} \mathrm{D}_{2}^{\circ} \\
z^{7} \mathrm{D}_{1}^{\circ}-f{ }^{5} \mathrm{~F}_{2} \\
a^{3} \mathrm{~F}_{2}-v{ }^{5} \mathrm{D}_{2}^{\circ}\end{array}$ & $\begin{array}{r}195 \\
259 \\
161 \\
53\end{array}$ & $\begin{array}{l}\text { Pred } \\
\text { New } \\
\text { Pred } \\
\text { SS } \\
\text { Pred }\end{array}$ & $\begin{array}{c}\mathrm{Z} \\
\mathrm{U} \\
\mathrm{U} \\
\mathrm{Z}, \mathrm{ZZ}\end{array}$ \\
\hline $\begin{array}{l}3150.762 \\
3149.492 \\
3148.676 \\
3148.178 \\
3144.924\end{array}$ & $\begin{array}{l}1 \\
1 \\
0 \\
1 n \\
1\end{array}$ & $\begin{array}{l}31729.17 \\
31741.96 \\
31750.18 \\
31755.21 \\
31788.06\end{array}$ & $\begin{array}{l}\text { 9. } 20 \\
\text { 1. } 93 \\
0.08 \\
\text { 5. } 36 \\
\text { 8. } 14\end{array}$ & $\begin{array}{l}a^{1} \mathrm{H}_{5}-t{ }^{3} \mathrm{H}_{5}^{\circ} \\
b^{3} \mathrm{G}_{5}-x \\
a^{1} \mathrm{H}_{5}^{\circ} \mathrm{P}_{2}-z{ }^{1} \mathrm{D}_{2}^{\circ} \\
a^{3} \mathrm{G}_{3}-11_{3}^{\circ} \\
a^{3} \mathrm{H}_{5}-4_{4}^{\circ}\end{array}$ & $\begin{array}{l}813 \\
453\end{array}$ & $\begin{array}{l}\text { New } \\
\text { Pred } \\
\text { New } \\
\text { New } \\
\text { Pred }\end{array}$ & $\begin{array}{l}\mathrm{U} \\
\mathrm{U} \\
\mathrm{U} \\
\mathrm{U}\end{array}$ \\
\hline
\end{tabular}


TABLE 3. Classified faint lines of Fe I-Continued

\begin{tabular}{|c|c|c|c|c|c|c|c|}
\hline \multirow{2}{*}{$\begin{array}{c}\text { Wavelength } \\
\text { A }\end{array}$} & \multirow{2}{*}{ Intensity } & \multicolumn{2}{|c|}{ Wave number $\left(\mathrm{cm}^{-1}\right)$} & \multirow{2}{*}{ Designation } & \multirow{2}{*}{$\begin{array}{l}\text { Multiplet } \\
\text { Number }\end{array}$} & \multirow{2}{*}{ Notes } & \multirow{2}{*}{ Reference } \\
\hline & & Observed & Calc. & & & & \\
\hline $\begin{array}{l}3138.400 \\
3135.590 \\
3134.641 \\
3134.401 \\
3133.174\end{array}$ & $\begin{array}{l}0 \\
1 n \\
0 \\
1 \\
0\end{array}$ & $\begin{array}{l}\text { 31854. } 14 \\
31882.69 \\
31892.34 \\
31894.78 \\
\text { 31907. } 27\end{array}$ & $\begin{array}{l}\text { 4. } 18 \\
\text { 2. } 74 \\
\text { 2. } 37 \\
\text { 4. } 67 \\
\text { 7. } 15\end{array}$ & $\begin{array}{l}a^{3} \mathrm{~F}_{3}-v{ }^{5} \mathrm{D}_{4}^{\circ} \\
a^{3} \mathrm{G}_{4}-53882_{4}^{\circ} \\
a^{3} \mathrm{G}_{4}-10_{3}^{\circ} \\
a^{3} \mathrm{G}_{5}-53610_{4}^{\circ} \\
a^{5} \mathrm{P}_{3}-49457_{4}^{\circ} ?\end{array}$ & 53 & $\begin{array}{l}\text { Pred } \\
\text { New } \\
\text { New } \\
\text { New } \\
\text { New }\end{array}$ & $\begin{array}{l}\mathrm{Z} \\
\mathrm{U} \\
\mathrm{Z}\end{array}$ \\
\hline $\begin{array}{l}3131.238 \\
3126.822 \\
3125.012 \\
3123.545 \\
3121.151\end{array}$ & $\begin{array}{l}0 \\
1 \\
1 \\
1 n \\
1\end{array}$ & $\begin{array}{l}31927.00 \\
31972.09 \\
31990.60 \\
32005.63 \\
32030.18\end{array}$ & $\begin{array}{l}\text { 6. } 89 \\
\text { 1. } 94 \\
\text { 0. } 49 \\
\text { 5. } 65 \\
0.17\end{array}$ & $\begin{array}{l}a^{5} \mathrm{P}_{3}-z{ }^{1} \mathrm{D}_{2}^{\circ} \\
b^{3} \mathrm{~F}_{4}-w^{3} \mathrm{H}_{5}^{\circ} \\
a^{3} \mathrm{~F}_{3}-v{ }^{5} \mathrm{D}_{3}^{\circ} \\
a^{3} \mathrm{G}_{4}-11_{3}^{\circ} \\
z^{7} \mathrm{D}_{1}^{\circ}-g{ }^{5} \mathrm{D}_{2}\end{array}$ & $\begin{array}{r}260 \\
53 \\
\\
163\end{array}$ & $\begin{array}{l}\text { New } \\
\text { Pred b } \\
\text { Pred } \\
\text { New } \\
\text { New }\end{array}$ & $\begin{array}{l}\mathrm{U} \\
\mathrm{U} \\
\mathrm{Z}\end{array}$ \\
\hline $\begin{array}{l}3120.220 \\
3119.032 \\
3116.984 \\
3116.502 \\
3116.379\end{array}$ & $\begin{array}{l}2 n \\
0 \\
1 n \\
1 n \\
1\end{array}$ & $\begin{array}{l}\text { 32039. } 73 \\
32051.94 \\
32072.99 \\
32077.95 \\
32079.22\end{array}$ & $\begin{array}{l}\text { 9. } 63 \\
\text { 1. } 90 \\
\text { 2. } 98 \\
\text { 7. } 8 \\
9.16\end{array}$ & $\begin{array}{l}a^{3} \mathrm{G}_{3}-54289_{3}^{\circ} \\
a^{3} \mathrm{G}_{3}-13_{4}^{\circ} \\
z^{5} \mathrm{D}_{3}^{\circ}-4_{2} \\
a^{5} \mathrm{P}_{2}-x \\
b^{7} \mathrm{P}_{3}^{\circ} ? \\
{ }^{3} \mathrm{~F}_{3}-s \\
{ }^{3} \mathrm{D}_{3}^{\circ}\end{array}$ & $\begin{array}{l}315 \mathrm{a} \\
578 \mathrm{a} \\
261\end{array}$ & $\begin{array}{l}\text { New } \\
\text { Pred } \\
\text { New } \\
\text { New } \\
\text { Pred b }\end{array}$ & $\underset{\mathrm{U}}{\mathrm{Z}} \underset{\mathrm{Z}}{\mathrm{Z}}, \mathrm{U}$ \\
\hline $\begin{array}{l}3115.862 \\
3115.656 \\
3114.054 \\
3113.592 \\
310 \% .978\end{array}$ & $\begin{array}{l}1 \\
2 \\
1 \\
2 \\
2 n\end{array}$ & $\begin{array}{l}32084.54 \\
32086.66 \\
32103.17 \\
32107.93 \\
32165.93\end{array}$ & $\begin{array}{l}\text { 4. } 53 \\
\text { 6. } 59 \\
\text { 3. } 18 \\
\text { 7. } 94 \\
\text { 6. } 13\end{array}$ & $\begin{array}{l}b^{3} \mathrm{G}_{3}-u{ }^{3} \mathrm{H}_{4}^{\circ} \\
c^{3} \mathrm{P}_{1}-u{ }^{3} \mathrm{~F}_{2}^{\circ} \\
a^{3} \mathrm{~F}_{3}-v{ }^{5} \mathrm{D}_{2}^{\circ} \\
a^{3} \mathrm{G}_{3}-54357_{3}^{\circ} \\
a^{3} \mathrm{G}_{5}-53882_{4}^{\circ}\end{array}$ & $\begin{array}{r}456 \\
53\end{array}$ & $\begin{array}{l}\text { New } \\
\text { New } \\
\text { New } \\
\text { New } \\
\text { New }\end{array}$ & $\begin{array}{l}\mathrm{U} \\
\mathrm{Z} \\
\mathrm{Z} \\
\mathrm{Z}\end{array}$ \\
\hline $\begin{array}{l}\text { 3103. } 760 \\
\text { 3099. } 118 \\
\text { 3098. } 963 \\
3097.500 \\
3096.044\end{array}$ & $\begin{array}{l}1 \\
0 \\
1 \\
0 n \\
1\end{array}$ & $\begin{array}{l}32209.64 \\
32257.88 \\
32259.50 \\
32274.73 \\
32289.91\end{array}$ & $\begin{array}{l}\text { 9. } 63 \\
\text { 7. } 92 \\
\text { 9. } 46 \\
\text { 4. } 82 \\
\text { 9. } 92\end{array}$ & $\begin{array}{l}a^{3} \mathrm{P}_{1}-x{ }^{1} \mathrm{D}_{2}^{\circ} \\
b^{3} \mathrm{~F}_{4}-y{ }^{3} \mathrm{I}_{5}^{\circ} \\
a^{5} \mathrm{P}_{1}-w{ }^{3} \mathrm{P}_{2}^{\circ} \\
z^{7} \mathrm{D}_{4}^{\circ}-e{ }^{5} \mathrm{P}_{3} \\
a^{3} \mathrm{G}_{4}-54289^{\circ}\end{array}$ & $\begin{array}{l}102 \\
165\end{array}$ & $\begin{array}{l}\text { New } \\
\text { New } \\
\text { New } \\
\text { Pred } \\
\text { New }\end{array}$ & $\begin{array}{c}\mathrm{Z} \\
\mathrm{U}, \mathrm{Z} \\
\mathrm{Z}\end{array}$ \\
\hline $\begin{array}{l}3087.420 \\
3081.832 \\
3081.278 \\
3071.276 \\
3056.250\end{array}$ & $\begin{array}{l}1 n \\
1 \\
1 \\
1 \\
2\end{array}$ & $\begin{array}{l}32380.10 \\
32438.81 \\
32444.64 \\
32550.30 \\
32710.33\end{array}$ & $\begin{array}{l}9.99 \\
8.87 \\
\text { 4. } 53 \\
0.36 \\
0.37\end{array}$ & $\begin{array}{c}z^{5} \mathrm{D}_{3}^{\circ}-g \\
a^{3} \mathrm{~F}_{4}-v \mathrm{G}_{4} ? \\
b^{5} \mathrm{D}_{4}^{\circ} \mathrm{G}_{3}-u{ }^{3} \mathrm{~F}_{3}^{\circ} \\
b^{3} \mathrm{G}_{5}-u{ }^{3} \mathrm{H}_{6}^{\circ} \\
b^{3} \mathrm{~F}_{2}-5374_{4}^{\circ}\end{array}$ & $\begin{array}{r}53 \\
457 \\
456\end{array}$ & $\begin{array}{l}\text { New } \\
\text { Pred } \\
\text { New } \\
\text { New c } \\
\text { New }\end{array}$ & $\begin{array}{c}\mathrm{U}, \mathrm{W} \\
\mathrm{BK}, \mathrm{Z} \\
\mathrm{BK}, \mathrm{U} \\
\mathrm{BK}, \mathrm{Z} \\
\mathrm{BK}\end{array}$ \\
\hline $\begin{array}{l}3030.605 \\
3011.883 \\
3006.598 \\
2978.060 \\
2975.655\end{array}$ & $\begin{array}{l}2 \\
2 \\
0 \\
1 \\
0\end{array}$ & $\begin{array}{l}\text { 32987. } 11 \\
\text { 33192. } 15 \\
33250.49 \\
\text { 33569. } 11 \\
\text { 33596. } 24\end{array}$ & $\begin{array}{l}\text { 7. } 08 \\
\text { 2. } 08 \\
0.40 \\
9.25 \\
\text { 6. } 32\end{array}$ & $\begin{array}{l}a^{3} \mathrm{P}_{2}-v{ }^{3} \mathrm{~F}_{3}^{\circ} \\
a^{3} \mathrm{P}_{0}-z{ }^{1} \mathrm{P}_{1}^{\circ} \\
b^{3} \mathrm{~F}_{4}-10_{3}^{\circ} \\
a^{3} \mathrm{H}_{4}-53358_{3}^{\circ} ? \\
b^{3} \mathrm{~F}_{4}-t{ }^{3} \mathrm{G}_{4}^{\circ}\end{array}$ & $\begin{array}{r}145 \\
\text { UV } 135\end{array}$ & $\begin{array}{l}\text { Pred b } \\
\text { New } \\
\text { New c } \\
\text { New } \\
\text { New }\end{array}$ & $\begin{array}{c}\text { BK, Z } \\
\text { BK, U, W } \\
\text { BK } \\
\text { BK, W, Z } \\
\text { BK, U }\end{array}$ \\
\hline $\begin{array}{l}2964.196 \\
2958.462 \\
2951.356 \\
2949.688 \\
2947.116\end{array}$ & $\begin{array}{l}1 \\
1 \\
0 \\
0 n \\
0\end{array}$ & $\begin{array}{l}\text { 33726. } 11 \\
33791.48 \\
33872.83 \\
33891.98 \\
33921.56\end{array}$ & $\begin{array}{l}\text { 5. } 83 \\
\text { 1. } 55 \\
\text { 2. } 85 \\
\text { 2. } 12 \\
\text { 1. } 54\end{array}$ & 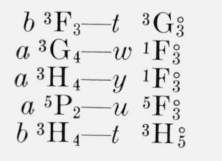 & $\begin{array}{r}317 \\
\text { UV117 } \\
\text { UV182 }\end{array}$ & $\begin{array}{l}\text { New } \\
\text { New } \\
\text { New } \\
\text { New } \\
\text { New }\end{array}$ & $\begin{array}{l}\text { BK, Z } \\
\text { BK, W, Z } \\
\text { BK, Z } \\
\text { BK, Z } \\
\text { BK, Z }\end{array}$ \\
\hline $\begin{array}{l}2946.095 \\
2945.870 \\
2945.050 \\
2931.803 \\
2924.002\end{array}$ & $\begin{array}{l}1 \\
0 \\
3 \\
1 \\
0\end{array}$ & $\begin{array}{l}\text { 33933. } 32 \\
\text { 33935. } 91 \\
33945.36 \\
34098.73 \\
34189.70\end{array}$ & $\begin{array}{l}\text { 3. } 32 \\
\text { 5. } 97 \\
\text { 5. } 23 \\
\text { 8. } 68 \\
\text { 9. } 79\end{array}$ & $\begin{array}{l}a^{3} \mathrm{~F}_{2}-y{ }^{3} \mathrm{P}_{1} \\
b^{3} \mathrm{H}_{4}-60564+3 \\
a^{3} \mathrm{H}_{4}-537344_{4}^{\circ} \\
a^{3} \mathrm{G}_{4}-s \\
{ }^{3} \mathrm{G}_{3}^{\circ} \\
{ }^{3} \mathrm{G}_{5}-s \\
{ }^{3} \mathrm{G}_{4}^{\circ}\end{array}$ & $\begin{array}{l}\text { UV166 } \\
\text { UV166 }\end{array}$ & $\begin{array}{l}\text { New } \\
\text { New c } \\
\text { New } \\
(\text { a) } \\
\text { New }\end{array}$ & $\begin{array}{c}\text { BK, Z } \\
\text { BK, Z } \\
\text { G } \\
\text { BK, W, Z } \\
\text { BK, Z }\end{array}$ \\
\hline $\begin{array}{l}\text { 2906. } 741 \\
2904.522 \\
2898.867 \\
\text { 2897. } 635 \\
2896.595\end{array}$ & $\begin{array}{l}0 \\
0 \\
\mathbf{1} \\
\mathbf{1} n \\
0\end{array}$ & $\begin{array}{l}34392.72 \\
34418.99 \\
34486.13 \\
34500.79 \\
34513.18\end{array}$ & $\begin{array}{l}\text { 2. } 74 \\
\text { 8. } 93 \\
\text { 6. } 27 \\
\text { 0. } 81 \\
\text { 3. } 08\end{array}$ & $\begin{array}{l}a^{3} \mathrm{H}_{5}-12_{5}^{\circ} \\
a^{5} \mathrm{P}_{3}-u^{3} \mathrm{D}_{3}^{\circ} \\
a^{5} \mathrm{P}_{2}-t{ }^{3} \mathrm{D}_{3}^{\circ} \\
a^{3} \mathrm{H}_{4}-54289_{3}^{\circ} \\
a^{3} \mathrm{H}_{4}-13_{4}^{\circ}\end{array}$ & UV150 & $\begin{array}{l}\text { New } \\
\text { New } \\
\text { New } \\
(a, e) \\
\text { New }\end{array}$ & $\begin{array}{c}\text { BK, U } \\
\text { BK } \\
\text { BK, W, Z } \\
\text { BK, } \underset{\text { BK, Z }}{ }\end{array}$ \\
\hline $\begin{array}{l}2891.904 \\
2891.705 \\
2890.414 \\
2882.634 \\
2879.741\end{array}$ & $\begin{array}{l}1 n \\
1 \\
1 \\
0 \\
0\end{array}$ & $\begin{array}{l}34569.16 \\
34571.54 \\
34586.98 \\
34680.32 \\
34715.16\end{array}$ & $\begin{array}{l}\text { 9. } 12 \\
\text { 1. } 54 \\
\text { 7. } 06 \\
\text { 0. } 32 \\
\text { 5. } 27\end{array}$ & $\begin{array}{c}a^{3} \mathrm{H}_{4}-54357^{\circ} \\
b^{3} \mathrm{~F}_{3}-v{ }^{3} \mathrm{H}_{4}^{\circ} \\
a^{3} \mathrm{~F}_{2}-y{ }^{3} \mathrm{~S}_{1}^{\circ} ? \\
a^{3} \mathrm{H}_{5}-13_{4}^{\circ} \\
b^{3} \mathrm{~F}_{2}-w^{1} \mathrm{D}_{2}^{\circ}\end{array}$ & UV158 & $\begin{array}{l}\text { New } \\
\text { New a, e } \\
\text { New } \\
\text { New } \\
\text { New a }\end{array}$ & $\begin{array}{c}\mathrm{V} \\
\mathrm{W}, \mathrm{Z} \\
\mathrm{BK}, \mathrm{Z} \\
\mathrm{BK}, \mathrm{U}\end{array}$ \\
\hline
\end{tabular}


TABLE 3. Classified faint lines of Fe I-Continued

\begin{tabular}{|c|c|c|c|c|c|c|c|}
\hline \multirow{2}{*}{$\begin{array}{c}\text { Wavelength } \\
\text { A }\end{array}$} & \multirow{2}{*}{ Intensity } & \multicolumn{2}{|c|}{ Wave number $\left(\mathrm{cm}^{-1}\right)$} & \multirow{2}{*}{ Designation } & \multirow{2}{*}{$\begin{array}{l}\text { Multiplet } \\
\text { Number }\end{array}$} & \multirow{2}{*}{ Notes } & \multirow{2}{*}{ Reference } \\
\hline & & Observed & Calc. & & & & \\
\hline $\begin{array}{l}2878.962 \\
2878.762 \\
2876.725 \\
2866.385 \\
2861.996\end{array}$ & $\begin{array}{l}1 \\
1 \\
1 \\
1 \\
0\end{array}$ & $\begin{array}{l}34724.55 \\
34726.97 \\
34751.56 \\
34876.91 \\
34930.39\end{array}$ & $\begin{array}{l}\text { 4. } 72 \\
\text { 6. } 99 \\
\text { 1. } 70 \\
\text { 6. } 99 \\
0.43\end{array}$ & $\begin{array}{c}a^{3} \mathrm{~F}_{2}-w^{5} \mathrm{G}_{3}^{\circ} \\
b^{3} \mathrm{P}_{2}-57565_{3}^{\circ} ? \\
b^{3} \mathrm{~F}_{2}-w^{1} \mathrm{~F}_{3}^{\circ} ? \\
a^{3} \mathrm{G}_{5}-u^{3} \mathrm{~F}_{4}^{\circ} \\
a^{5} \mathrm{P}_{1}-x{ }^{3} \mathrm{~S}_{1}^{\circ}\end{array}$ & $\begin{array}{l}\text { UV90 } \\
\text { UV168 }\end{array}$ & $\begin{array}{l}\text { New } \\
\text { New } \\
\text { New } \\
\text { New } \\
\text { New }\end{array}$ & $\begin{array}{c}\text { BK, } \mathrm{U}, \mathrm{W} \\
\mathrm{V} \\
\mathrm{BK}, \mathrm{Z} \\
\mathrm{BK}, \mathrm{U} \\
\text { BK }\end{array}$ \\
\hline $\begin{array}{l}2860.206 \\
2857.996 \\
2830.754 \\
2819.462 \\
2815.836\end{array}$ & $\begin{array}{l}0 n \\
1 n \\
0 \\
1 \\
0\end{array}$ & $\begin{array}{l}34952.25 \\
34979.28 \\
35315.89 \\
35457.32 \\
35502.98\end{array}$ & $\begin{array}{l}\text { 2. } 10 \\
9.31 \\
\text { 5. } 89 \\
\text { 7. } 24 \\
\text { 2. } 92\end{array}$ & $\begin{array}{l}a^{3} \mathrm{G}_{4}-v{ }^{1} \mathrm{G}_{4}^{\circ} \\
a^{3} \mathrm{P}_{2}-53358_{3}^{\circ} \\
a^{3} \mathrm{G}_{3}-57565_{3}^{\circ} ? \\
a^{3} \mathrm{D}_{2}-62081_{2}^{\circ} ? \\
a^{5} \mathrm{P}_{2}-z{ }^{1} \mathrm{P}_{1}^{\circ}\end{array}$ & & $\begin{array}{l}\text { New } \\
\text { New } \\
\text { New } \\
(a, e) \\
\text { New }\end{array}$ & $\begin{array}{c}\text { BK, Z } \\
\text { Z } \\
\text { BK, U, W } \\
\text { BK, W, Z } \\
\text { BK, U }\end{array}$ \\
\hline $\begin{array}{l}2810.834 \\
2802.285 \\
2797.046 \\
2790.762 \\
2783.560\end{array}$ & $\begin{array}{l}1 \\
0 n \\
0 \\
0 \\
0\end{array}$ & $\begin{array}{l}35566.15 \\
35674.65 \\
35741.47 \\
35821.94 \\
35914.62\end{array}$ & $\begin{array}{l}\text { 6. } 18 \\
\text { 4. } 78 \\
\text { 1. } 55 \\
\text { 1. } 98 \\
\text { 4. } 79\end{array}$ & $\begin{array}{l}a^{3} \mathrm{G}_{4}-57565_{3}^{\circ} \\
a^{3} \mathrm{D}_{1}-62081_{2}^{\circ} ? \\
b^{3} \mathrm{~F}_{4}-u^{3} \mathrm{H}_{5}^{\circ} \\
a^{5} \mathrm{P}_{1}-5379_{2}^{\circ} \\
a^{3} \mathrm{~F}_{3}-w^{3} \mathrm{G}_{3}^{\circ}\end{array}$ & UV95 & $\begin{array}{l}\text { New } \\
\text { New } \\
\text { New c } \\
\text { New c } \\
\text { New }\end{array}$ & $\begin{array}{l}\text { U, W } \\
\text { BK, Z } \\
\text { BK } \\
\text { BK } \\
\text { BK }\end{array}$ \\
\hline $\begin{array}{l}2780.880 \\
2780.526 \\
2776.767 \\
2772.511 \\
2772.320\end{array}$ & $\begin{array}{l}1 \\
1 \\
1 \\
1 \\
1\end{array}$ & $\begin{array}{l}35949.23 \\
35953.81 \\
36002.48 \\
36057.74 \\
36060.23\end{array}$ & $\begin{array}{l}\text { 9. } 20 \\
\text { 3. } 78 \\
\text { 2. } 44 \\
\text { 7. } 72 \\
0.23\end{array}$ & $\begin{array}{c}a^{5} \mathrm{~F}_{4}-z{ }^{5} \mathrm{H}_{3}^{\circ} \\
a^{3} \mathrm{~F}_{4}-v \\
{ }^{5} \mathrm{~F}_{4}^{\circ} \\
a^{3} \mathrm{H}_{4}-w{ }^{1} \mathrm{~F}_{3}^{\circ} \\
a^{5} \mathrm{P}_{2}-53785_{3}^{\circ} \\
a^{5} \mathrm{P}_{3}-53610_{4}^{\circ}\end{array}$ & $\begin{array}{l}\text { UV45 } \\
\text { UV92 }\end{array}$ & $\begin{array}{l}\text { New } \\
\text { New } \\
\text { New c } \\
\text { New } \\
\text { New }\end{array}$ & $\begin{array}{c}\text { BK, Z } \\
\text { BK, Z } \\
\text { BK, U } \\
\text { V } \\
\text { BK, W, Z }\end{array}$ \\
\hline $\begin{array}{l}2766.560 \\
2760.623 \\
2759.500 \\
2758.993 \\
2751.808\end{array}$ & $\begin{array}{l}1 \\
1 \\
0 \\
1 \\
1\end{array}$ & $\begin{array}{l}36135.30 \\
36213.01 \\
36227.74 \\
36234.40 \\
36329.00\end{array}$ & $\begin{array}{l}\text { 5. } 38 \\
\text { 3. } 07 \\
\text { 7. } 81 \\
\text { 4. } 53 \\
9.09\end{array}$ & $\begin{array}{l}a{ }^{3} \mathrm{H}_{6}-x{ }^{1} \mathrm{H}_{5}^{\circ} \\
a^{5} \mathrm{P}_{3}-x{ }^{1} \mathrm{~F}_{3}^{\circ} \\
c^{3} \mathrm{P}_{2}-60564_{3}^{\circ} ? \\
a^{5} \mathrm{P}_{3}-53785_{3}^{\circ} \\
a^{3} \mathrm{~F}_{2}-v{ }^{3} \mathrm{D}_{1}^{\circ} ?\end{array}$ & $\begin{array}{l}\text { UV152 } \\
\text { UV127 }\end{array}$ & $\begin{array}{l}\text { New } \\
\text { New } \\
\text { New } \\
\text { New } \\
\text { New }\end{array}$ & $\begin{array}{c}\text { BK } \\
\text { BK } \\
\text { BK, U } \\
\text { BK, W } \\
\text { BK, W, Z }\end{array}$ \\
\hline $\begin{array}{l}\text { 2749. } 688 \\
2745.952 \\
2732.778 \\
2725.311 \\
2724.344\end{array}$ & $\begin{array}{l}0 \\
0 \\
1 \\
1 \\
1 n\end{array}$ & $\begin{array}{l}\text { 36357. } 01 \\
36406.48 \\
36581.97 \\
\text { 36682. } 20 \\
\text { 36695. } 22\end{array}$ & $\begin{array}{l}\text { 7. } 14 \\
6.37 \\
\text { 2. } 05 \\
\text { 2. } 00 \\
\text { 5. } 00\end{array}$ & $\begin{array}{l}a^{5} \mathrm{~F}_{1}-y{ }^{5} \mathrm{~S}_{2}^{\circ} \\
a^{3} \mathrm{~F}_{4}-z{ }^{1} \mathrm{H}_{5}^{\circ} \\
b^{3} \mathrm{G}_{5}-t{ }^{3} \mathrm{H}_{6}^{\circ} ? \\
a^{3} \mathrm{~F}_{3}-w^{3} \mathrm{~F}_{3}^{\circ} \\
z^{7} \mathrm{D}_{3}^{\circ}-2_{4}\end{array}$ & $\begin{array}{l}\text { UV49 } \\
\text { UV98 } \\
\text { UV144 }\end{array}$ & $\begin{array}{l}\text { New } \\
\text { New } \\
\text { New } \\
\text { New } \\
\text { New }\end{array}$ & $\begin{array}{c}\text { BK, Z } \\
\text { BK, Z } \\
\text { BK } \\
\text { BK, W, Z } \\
\text { BK, U }\end{array}$ \\
\hline $\begin{array}{l}2723.032 \\
270 \% .451 \\
2679.513 \\
2667.22 \\
2648.548\end{array}$ & $\begin{array}{l}0 \\
2 \\
0 \\
1 \\
1\end{array}$ & $\begin{array}{l}36712.90 \\
36924.16 \\
37309.13 \\
37481.08 \\
37745.30\end{array}$ & $\begin{array}{l}\text { 2. } 97 \\
\text { 4. } 21 \\
\text { 9. } 21 \\
\text { 1. } 10 \\
\text { 5. } 47\end{array}$ & $\begin{array}{c}a{ }^{3} \mathrm{H}_{5}-u^{3} \mathrm{H}_{6}^{\circ} \\
b{ }^{3} \mathrm{~F}_{4}-57565_{3}^{\circ} \\
c^{3} \mathrm{P}_{1}-62081_{2}^{\circ} ? \\
a^{3} \mathrm{~F}_{4}-4945 \gamma_{4}^{\circ} ? \\
c^{3} \mathrm{P}_{2}-62081_{2}^{\circ} ?\end{array}$ & UV154 & $\begin{array}{l}\text { New } \\
\text { New } \\
\text { New } \\
\text { New c } \\
\text { New }\end{array}$ & $\begin{array}{c}\mathrm{BK} \\
\mathrm{Z} \\
\mathrm{BK}, \mathrm{U} \\
\mathrm{U} \\
\mathrm{BK}, \mathrm{W}, \mathrm{Z}\end{array}$ \\
\hline $\begin{array}{l}\text { 2648. } 164 \\
\text { 2647. } 390 \\
\text { 2642. } 274 \\
\text { 2641. } 031 \\
\text { 2627. } 230\end{array}$ & $\begin{array}{l}1 \\
1 \\
0 \\
1 \\
1\end{array}$ & $\begin{array}{l}37750.77 \\
37761.81 \\
37834.92 \\
37852.72 \\
38051.55\end{array}$ & $\begin{array}{l}0.80 \\
\text { 1. } 81 \\
\text { 4. } 96 \\
\text { 2. } 78 \\
\text { 1. } 68\end{array}$ & 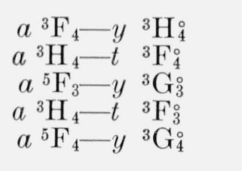 & $\begin{array}{l}\text { UV99 } \\
\text { UV51 } \\
\text { UV51 }\end{array}$ & $\begin{array}{l}\text { New } \\
\text { New } \\
\text { New } \\
\text { New } \\
\text { New }\end{array}$ & $\begin{array}{l}\text { BK, W, Z } \\
\text { BK, U } \\
\text { BK, U } \\
\text { BK, U } \\
\text { BK, Z }\end{array}$ \\
\hline $\begin{array}{l}\text { 2627. } 118 \\
2609.220 \\
2603.042 \\
2596.618 \\
2596.077\end{array}$ & $\begin{array}{l}1 \\
2 \\
0 \\
0 \\
1\end{array}$ & $\begin{array}{l}38053.18 \\
38314.19 \\
38405.12 \\
38500.12 \\
38508.15\end{array}$ & $\begin{array}{l}\text { 3. } 06 \\
\text { 4. } 15 \\
5.11 \\
0.18 \\
8.22\end{array}$ & $\begin{array}{l}a^{3} \mathrm{~F}_{3}-x{ }^{1} \mathrm{G}_{4}^{\circ} \\
a^{3} \mathrm{G}_{3}-60564_{4}^{\circ} \\
a^{3} \mathrm{P}_{2}-u{ }^{3} \mathrm{~F}_{3}^{\circ} \\
a^{5} \mathrm{~F}_{5}-y{ }^{3} \mathrm{G}_{4}^{\circ} \\
a^{3} \mathrm{G}_{3}-t{ }^{3} \mathrm{H}_{4}^{\circ}\end{array}$ & $\begin{array}{l}\text { UV51 } \\
\text { UV171 }\end{array}$ & $\begin{array}{l}\text { New } \\
\text { New } \\
\text { New } \\
\text { New } \\
\text { New }\end{array}$ & $\begin{array}{c}\text { BK, W, Z } \\
\text { G } \\
\text { BK } \\
\text { BK, U } \\
\text { BK, U }\end{array}$ \\
\hline $\begin{array}{l}2593.268 \\
2592.285 \\
2588.898 \\
2580.939 \\
2580.561\end{array}$ & $\begin{array}{l}0 \\
3 \\
0 \\
1 \\
1\end{array}$ & $\begin{array}{l}38549.86 \\
38564.47 \\
38614.92 \\
38733.99 \\
38739.67\end{array}$ & $\begin{array}{l}0.01 \\
\text { 4. } 44 \\
\text { 5. } 09 \\
\text { 3. } 86 \\
9.76\end{array}$ & $\begin{array}{l}a^{3} \mathrm{G}_{4}-t{ }^{3} \mathrm{H}_{5}^{\circ} \\
a^{3} \mathrm{G}_{4}-60564{ }^{\circ} \\
a^{5} \mathrm{~F}_{2}-z \\
a^{3} \mathrm{~S}_{1}^{\circ} \\
a^{5} \mathrm{~F}_{1}-u{ }^{5} \mathrm{D}_{2}^{\circ} \\
a^{3} \mathrm{~F}_{2}-y{ }^{1} \mathrm{D}_{2}^{\circ}\end{array}$ & $\begin{array}{l}\text { UV171 } \\
\text { UV55 }\end{array}$ & $\begin{array}{l}\text { New } \\
\text { New } \\
\text { New } \\
\text { New } \\
\text { New c }\end{array}$ & $\begin{array}{l}\text { BK, U } \\
\text { G } \\
\text { BK, U } \\
\text { BK, Z } \\
\text { BK, U }\end{array}$ \\
\hline $\begin{array}{l}2580.281 \\
2554.518 \\
2550.812 \\
2547.468 \\
2546.176\end{array}$ & $\begin{array}{l}0 \\
1 \\
1 \\
0 \\
1\end{array}$ & $\begin{array}{l}38743.87 \\
39134.59 \\
39191.44 \\
39242.88 \\
39262.80\end{array}$ & $\begin{array}{l}\text { 3. } 70 \\
\text { 4. } 41 \\
\text { 1. } 45 \\
\text { 2. } 91 \\
\text { 2. } 84\end{array}$ & $\begin{array}{l}a^{3} \mathrm{~F}_{3}-v{ }^{3} \mathrm{~F}_{4}^{\circ} ? \\
b^{3} \mathrm{P}_{1}-62081_{2}^{\circ} ? \\
a^{5} \mathrm{~F}_{2}-u{ }^{5} \mathrm{D}_{1}^{\circ} \\
b^{3} \mathrm{P}_{2}-62081_{2}^{\circ} ? \\
a^{3} \mathrm{P}_{2}-t{ }^{3} \mathrm{~F}_{3}^{\circ}\end{array}$ & UV55 & $\begin{array}{l}\text { New } \\
\text { New } \\
\text { New } \\
\text { New } \\
\text { New }\end{array}$ & $\begin{array}{l}\text { BK, Z } \\
\text { G } \\
\text { BK, Z } \\
\text { BK, U } \\
\text { BK, U }\end{array}$ \\
\hline $\begin{array}{l}\text { 2544. } 462 \\
2538.693 \\
2529.306 \\
2518.824 \\
2512.266\end{array}$ & $\begin{array}{l}1 \\
1 \\
2 \\
2 \\
3\end{array}$ & $\begin{array}{l}39289.24 \\
39378.52 \\
39524.66 \\
39689.12 \\
39792.72\end{array}$ & $\begin{array}{l}9.17 \\
8.47 \\
\text { 4. } 59 \\
9.09 \\
\text { 2. } 57\end{array}$ & $\begin{array}{c}a^{5} \mathrm{~F}_{3}-w^{3} \mathrm{D}_{3}^{\circ} \\
a^{5} \mathrm{~F}_{3}-z{ }^{3} \mathrm{H}_{4}^{\circ} \\
\left(b^{3} \mathrm{~F}_{2}-605644_{3}^{\circ}\right) ? \\
b^{3} \mathrm{~F}_{3}-60564_{3}^{\circ} \\
a^{5} \mathrm{~F}_{5}-u^{5} \mathrm{D}_{4}^{\circ}\end{array}$ & $\begin{array}{l}\text { UV58 } \\
\text { UV57 } \\
\text { UV55 }\end{array}$ & $\begin{array}{l}\text { New } \\
\text { New c } \\
\text { New f } \\
\text { New } \\
\text { New }\end{array}$ & $\begin{array}{c}\text { BK, U } \\
\text { BK, Z } \\
\text { Z } \\
\text { Z } \\
\text { BK, U }\end{array}$ \\
\hline
\end{tabular}


TABLE 3. Classified faint lines of Fe I-Continued

\begin{tabular}{|c|c|c|c|c|c|c|c|}
\hline \multirow{2}{*}{$\begin{array}{c}\text { Wavelength } \\
\text { A }\end{array}$} & \multirow{2}{*}{ Intensity } & \multicolumn{2}{|c|}{ Wave number $\left(\mathrm{cm}^{-1}\right)$} & \multirow{2}{*}{ Designation } & \multirow{2}{*}{$\begin{array}{l}\text { Multiplet } \\
\text { Number }\end{array}$} & \multirow{2}{*}{ Notes } & \multirow{2}{*}{ Reference } \\
\hline & & Observed & Calc. & & & & \\
\hline $\begin{array}{l}2509.390 \\
2508.948 \\
2504.635 \\
2504.101 \\
2499.693\end{array}$ & $\begin{array}{l}1 \\
1 \\
1 \\
1 \\
1\end{array}$ & $\begin{array}{l}\text { 39838. } 32 \\
39845.34 \\
39913.95 \\
39922.46 \\
39992.86\end{array}$ & $\begin{array}{l}\text { 8. } 33 \\
5.40 \\
\text { 4. } 04 \\
\text { 2. } 47 \\
\text { 2. } 88\end{array}$ & $\begin{array}{l}a^{5} \mathrm{P}_{2}-57565_{3}^{\circ} \\
a^{5} \mathrm{~F}_{2}-w^{5} \mathrm{G}_{2}^{\circ} \\
a^{5} \mathrm{P}_{2}-t^{3} \mathrm{~F}_{3}^{\circ} \\
b^{3} \mathrm{~F}_{4}-6056_{43} \\
a^{3} \mathrm{~F}_{4}-u^{3} \mathrm{D}_{3}^{\circ}\end{array}$ & $\begin{array}{l}\text { UV59 } \\
\text { UV104 }\end{array}$ & $\begin{array}{l}\text { New } \\
\text { New } \\
\text { New } \\
\text { New } \\
\text { New }\end{array}$ & $\begin{array}{l}\text { BK, U } \\
\text { BK, Z } \\
\text { BK, U } \\
\text { BK, Z } \\
\text { BK, U }\end{array}$ \\
\hline $\begin{array}{l}2498.698 \\
2494.504 \\
2492.822 \\
2489.917 \\
2484.530\end{array}$ & $\begin{array}{l}1 \\
1 \\
1 \\
1 \\
1\end{array}$ & $\begin{array}{l}\text { 40008. } 78 \\
\text { 40076. } 04 \\
\text { 40103. } 08 \\
\text { 40149. } 87 \\
\text { 40236. } 92\end{array}$ & $\begin{array}{l}\text { 8. } 77 \\
\text { 6. } 00 \\
\text { 3. } 13 \\
\text { 9. } 98 \\
\text { 7. } 03\end{array}$ & 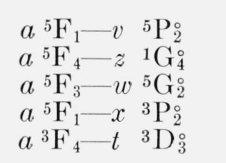 & $\begin{array}{l}\text { UV61 } \\
\text { UV59 } \\
\text { UV65 }\end{array}$ & $\begin{array}{l}\text { New } \\
\text { New } \\
\text { New } \\
\text { New } \\
\text { New c }\end{array}$ & $\begin{array}{l}\mathrm{BK}, \mathrm{U} \\
\mathrm{BK}, \mathrm{U} \\
\mathrm{BK}, \mathrm{U} \\
\mathrm{BK} \\
\mathrm{BK}, \mathrm{Z}\end{array}$ \\
\hline $\begin{array}{l}2480.393 \\
2469.666 \\
2466.530 \\
2460.069 \\
2456.704\end{array}$ & $\begin{array}{l}1 \\
1 \\
1 \\
1 \\
1\end{array}$ & $\begin{array}{l}40304.02 \\
40479.07 \\
40530.53 \\
40636.97 \\
40692.63\end{array}$ & $\begin{array}{l}\text { 4. } 09 \\
\text { 9. } 09 \\
\text { 0. } 35 \\
\text { 6. } 82 \\
\text { 2. } 56\end{array}$ & 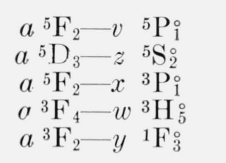 & $\begin{array}{l}\text { UV10 } \\
\text { UV65 } \\
\text { UV106 }\end{array}$ & $\begin{array}{l}\text { New } \\
\text { New } \\
\text { New } \\
\text { New } \\
\text { New }\end{array}$ & $\begin{array}{l}\mathrm{BK}, \mathrm{U} \\
\mathrm{BK}, \mathrm{U} \\
\mathrm{BK}, \mathrm{U} \\
\mathrm{BK}, \mathrm{U} \\
\text { BK }\end{array}$ \\
\hline $\begin{array}{l}2453.568 \\
2452.345 \\
2452.172 \\
2451.384 \\
2450.439\end{array}$ & $\begin{array}{l}3 \\
1 \\
2 \\
2 \\
3\end{array}$ & $\begin{array}{l}40744.64 \\
40764.95 \\
40767.83 \\
40780.93 \\
40796.66\end{array}$ & $\begin{array}{l}\text { 4. } 66 \\
\text { 4. } 94 \\
\text { 7. } 92 \\
\text { 0. } 82 \\
\text { 6. } 58\end{array}$ & $\begin{array}{l}a^{3} \mathrm{H}_{5}-t{ }^{3} \mathrm{H}_{6}^{\circ} \\
a^{3} \mathrm{~F}_{2}-53734_{4}^{\circ} \\
a^{3} \mathrm{~F}_{3}-9_{4}^{\circ} \\
a^{3} \mathrm{~F}_{2}-53749_{2}^{\circ} \\
a^{3} \mathrm{~F}_{3}-53358_{3}^{\circ}\end{array}$ & $\begin{array}{l}\text { UV } 157 \\
\text { UV105 }\end{array}$ & $\begin{array}{l}\text { New } \\
\text { New } \\
\text { New } \\
\text { New c } \\
\text { New }\end{array}$ & $\begin{array}{l}\text { BK, U } \\
\text { BK, U } \\
\text { BK, U } \\
\text { G } \\
\text { G }\end{array}$ \\
\hline $\begin{array}{l}2448.570 \\
2444.905 \\
2439.630 \\
2439.169 \\
2433.056\end{array}$ & $\begin{array}{l}0 \\
1 \\
3 \\
1 \\
2\end{array}$ & $\begin{array}{l}\text { 40827. } 80 \\
40889.00 \\
40977.40 \\
40985.14 \\
41088.11\end{array}$ & $\begin{array}{l}\text { 7. } 73 \\
8.95 \\
7.42 \\
\text { 5. } 14 \\
7.96\end{array}$ & 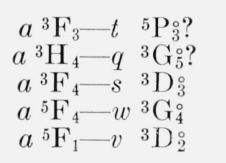 & $\begin{array}{l}\text { UV64 } \\
\text { UV68 }\end{array}$ & $\begin{array}{l}\text { New } \\
\text { New } \\
\text { New } \\
\text { New } \\
\text { New c }\end{array}$ & $\begin{array}{l}\text { BK } \\
\text { BK, U } \\
\text { BK, U } \\
\text { BK, U } \\
\text { BK, U }\end{array}$ \\
\hline $\begin{array}{l}\text { 2432. } 402 \\
2432.332 \\
2430.192 \\
2429.431 \\
2426.313\end{array}$ & $\begin{array}{l}0 \\
1 \\
2 \\
1 \\
1 n\end{array}$ & $\begin{array}{l}\text { 41099. } 16 \\
41100.34 \\
41136.53 \\
41149.41 \\
41202.29\end{array}$ & $\begin{array}{l}\text { 8. } 96 \\
0.18 \\
\text { 6. } 64 \\
\text { 9. } 28 \\
\text { 2. } 33\end{array}$ & 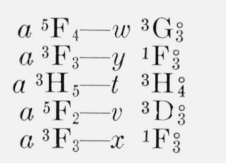 & $\begin{array}{l}\text { UV64 } \\
\text { UV106 } \\
\text { UV157 } \\
\text { UV68 }\end{array}$ & $\begin{array}{l}\text { New } \\
\text { New } \\
\text { New o } \\
\text { New } \\
\text { New }\end{array}$ & $\begin{array}{c}\text { BK } \\
\text { BK } \\
\text { BK, Z } \\
\text { BK, W } \\
\text { BK, Z }\end{array}$ \\
\hline $\begin{array}{l}2414.318 \\
2412.766 \\
2412.172 \\
2411.968 \\
2411.558\end{array}$ & $\begin{array}{l}0 \\
1 \\
0 \\
1 \\
1\end{array}$ & $\begin{array}{l}41406.98 \\
41433.61 \\
41443.81 \\
41447.32 \\
41454.36\end{array}$ & $\begin{array}{l}\text { 7. } 01 \\
\text { 3. } 64 \\
\text { 3. } 87 \\
\text { 7. } 38 \\
\text { 4. } 35\end{array}$ & $\begin{array}{l}a^{5}{ }^{5} \mathrm{~F}_{3}-v \\
a^{5} \mathrm{r}_{5}{ }^{3} \mathrm{D}_{3}^{\circ} \\
a^{3}{ }^{3} \mathrm{~F}_{3}-11_{3}^{\circ} \\
a^{5} \mathrm{~F}_{2}-w^{3} \mathrm{~F}_{2}^{\circ} \\
a^{5} \mathrm{~F}_{5}-z{ }^{1} \mathrm{H}_{5}^{\circ}\end{array}$ & $\begin{array}{l}\text { UV68 } \\
\text { UV64 } \\
\text { UV67 }\end{array}$ & $\begin{array}{l}\text { New } \\
\text { New } \\
\text { New } \\
\text { New } \\
\text { New }\end{array}$ & $\begin{array}{l}\text { BK } \\
\text { BK } \\
\text { BK, U } \\
\text { BK, U } \\
\text { BK }\end{array}$ \\
\hline $\begin{array}{l}2401.136 \\
2394.058 \\
2393.094 \\
2391.826 \\
2387.830\end{array}$ & $\begin{array}{l}1 \\
0 \\
1 \\
1 \\
1\end{array}$ & $\begin{array}{l}\text { 41634. } 28 \\
41757.36 \\
41774.18 \\
\text { 41796. } 33 \\
41866.27\end{array}$ & $\begin{array}{l}\text { 4. } 18 \\
\text { 7. } 25 \\
\text { 4. } 29 \\
\text { 6. } 45 \\
\text { 6. } 18\end{array}$ & $\begin{array}{l}a^{3}{ }^{3} \mathbf{F}_{4}-53610_{4}^{\circ} \\
a^{3} \mathbf{F}_{4}-53734^{\circ} \\
a^{5} \mathbf{F}_{5}-y{ }^{1} \mathrm{G}_{4}^{\circ} \\
a^{3} \mathbf{F}_{3}-54357^{\circ} \\
a^{5} \mathbf{F}_{4}-w^{3} \mathbf{F}_{3}^{\circ}\end{array}$ & $\begin{array}{l}\text { UV } 66 \\
\text { UV } 67\end{array}$ & $\begin{array}{l}\text { New } \\
\text { New } \\
\text { New } \\
\text { New } \\
\text { New }\end{array}$ & $\begin{array}{c}\text { BK } \\
\text { BK } \\
\text { BK } \\
\text { BK, Z } \\
\text { BK, U }\end{array}$ \\
\hline $\begin{array}{l}2385.580 \\
2378.604 \\
2376.971 \\
2375.678 \\
2367.394\end{array}$ & $\begin{array}{l}1 \\
1 \\
0 \\
0 \\
0\end{array}$ & $\begin{array}{l}41905.75 \\
42028.64 \\
42057.51 \\
42080.40 \\
42227.64\end{array}$ & $\begin{array}{l}\text { 5. } 65 \\
\text { 8. } 56 \\
\text { 7. } 45 \\
\text { 0. } 58 \\
\text { 7. } 67\end{array}$ & $\begin{array}{l}a^{3} \mathbf{F}_{4}-53882_{4}^{\circ} \\
a^{3} \mathbf{F}_{4}-11_{3}^{\circ} \\
a^{5} \mathbf{F}_{2}-w^{3} \mathrm{P}_{1}^{\circ} ? \\
a^{5} \mathbf{F}_{4}-49457^{\circ} \\
a^{5} \mathbf{F}_{4}-y^{3} \mathbf{H}_{5}^{\circ}\end{array}$ & & $\begin{array}{l}\text { New } \\
\text { New } \\
\text { New } \\
\text { New } \\
\text { New }\end{array}$ & $\begin{array}{l}\mathrm{BK}, \mathrm{Z} \\
\mathrm{BK} \\
\mathrm{BK} \\
\mathrm{BK}, \mathrm{U} \\
\mathrm{BK}\end{array}$ \\
\hline $\begin{array}{l}2362.624 \\
2361.936 \\
2356.196\end{array}$ & $\begin{array}{l}1 \\
1 \\
1\end{array}$ & $\begin{array}{l}42312.89 \\
42325.21 \\
42428.31\end{array}$ & $\begin{array}{l}\text { 2. } 83 \\
\text { 5. } 10 \\
8.1\end{array}$ & $\begin{array}{l}a^{3} \mathbf{F}_{4}-54289_{3}^{\circ} \\
a^{3} \mathbf{F}_{4}-13_{4}^{\circ} \\
a^{5} \mathbf{F}_{4}-x{ }^{7} \mathrm{P}_{3}^{\circ} ?\end{array}$ & & $\begin{array}{l}\text { New } \\
\text { New } \\
\text { New }\end{array}$ & $\begin{array}{l}\text { BK } \\
\text { BK } \\
\text { BK }\end{array}$ \\
\hline 2351.884 & 1 & 42506. 10 & $\begin{array}{l}\text { 5. } 92 \\
6.04\end{array}$ & $\begin{array}{l}a^{5}{ }^{5} \mathrm{~F}_{5}-y^{3} \mathrm{H}_{6}^{\circ} \\
a^{5} \mathrm{D}_{2}-y^{5} \mathrm{G}_{2}^{0}\end{array}$ & UV12 & New & $\mathrm{BK}$ \\
\hline 2350.626 & 1 & 42528. 84 & 8. 78 & $a^{3} \mathrm{P}_{1}-62081_{2}^{\circ} ?$ & & New & $\mathrm{BK}$ \\
\hline $\begin{array}{l}2346.304 \\
2345.018 \\
2331.088 \\
2323.627 \\
2296.188\end{array}$ & $\begin{array}{l}1 \\
1 n \\
1 \\
1 \\
2\end{array}$ & $\begin{array}{l}\text { 42607. } 18 \\
42630.54 \\
42885.27 \\
43022.96 \\
43537.03\end{array}$ & $\begin{array}{l}\text { 7. } 06 \\
0.2 \\
\text { 5. } 11 \\
\text { 3. } 00 \\
\text { 6. } 90\end{array}$ & 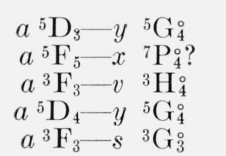 & $\begin{array}{l}\text { UV12 } \\
\text { UV108 } \\
\text { UV12 } \\
\text { UV111 }\end{array}$ & $\begin{array}{l}\text { New c } \\
\text { New } \\
\text { New c } \\
\text { New } \\
\text { New }\end{array}$ & $\begin{array}{c}\text { BK } \\
\text { BK, U, W } \\
\text { BK } \\
\text { BK, U } \\
B K, X, Z\end{array}$ \\
\hline
\end{tabular}


TABLE 3. Classified faint lines of Fe I-Concluded

\begin{tabular}{|c|c|c|c|c|c|c|c|}
\hline \multirow{2}{*}{$\begin{array}{c}\text { Wavelength } \\
\text { A }\end{array}$} & \multirow{2}{*}{ Intensity } & \multicolumn{2}{|c|}{ Wave number $\left(\mathrm{cm}^{-1}\right)$} & \multirow{2}{*}{ Designation } & \multirow{2}{*}{$\begin{array}{l}\text { Multiplet } \\
\text { Number }\end{array}$} & \multirow{2}{*}{ Notes } & \multirow{2}{*}{ Reference } \\
\hline & & Observed & Calc. & & & & \\
\hline $\begin{array}{l}2295.310 \\
2294.100 \\
2290.907 \\
2288.608 \\
2287.462\end{array}$ & $\begin{array}{l}1 \\
3 \\
3 \\
1 \\
3\end{array}$ & $\begin{array}{l}\text { 43553. } 68 \\
43576.65 \\
43637.38 \\
43681.21 \\
43703.09\end{array}$ & $\begin{array}{l}\text { 3. } 61 \\
6.58 \\
\text { 7. } 23 \\
\text { 1. } 11 \\
\text { 3. } 05\end{array}$ & $\begin{array}{l}a^{5} \mathrm{~F}_{1}-y{ }^{1} \mathrm{D}_{2}^{\circ} \\
a^{5} \mathrm{~F}_{3}-v{ }^{3} \mathrm{~F}_{4}^{\circ} \\
a^{5} \mathrm{~F}_{3}-v \\
a^{3} \mathrm{~F}_{3}^{\circ} \mathrm{F}_{3}-4 \\
a^{\circ}{ }^{3} \mathrm{P}_{2}-62081_{2}^{\circ} ?\end{array}$ & UV72 & $\begin{array}{l}\text { New } \\
\text { New } \\
\text { New } \\
\text { New } \\
\text { New }\end{array}$ & $\begin{array}{l}\text { BK, U } \\
\text { BK, U } \\
\text { BK } \\
\text { BK } \\
\text { BK, U }\end{array}$ \\
\hline $\begin{array}{l}2286.442 \\
2281.629 \\
2279.152 \\
2275.758 \\
2275.676\end{array}$ & $\begin{array}{l}3 \\
2 \\
2 \\
3 \\
1\end{array}$ & $\begin{array}{l}43722.59 \\
43814.81 \\
43862.42 \\
43927.83 \\
43929.42\end{array}$ & $\begin{array}{l}2.53 \\
4.76 \\
2.38 \\
7.87 \\
9.30\end{array}$ & 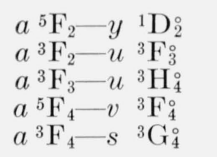 & $\begin{array}{l}\text { UV } 112 \\
\text { UV111 }\end{array}$ & $\begin{array}{l}\text { New } \\
\text { New a, e } \\
\text { New } \\
\text { New } \\
\text { New }\end{array}$ & $\begin{array}{l}\text { BK, Z } \\
\text { U, X } \\
\text { BK, U } \\
\text { BK, U }\end{array}$ \\
\hline $\begin{array}{l}2273.893 \\
2272.610 \\
2270.675 \\
2270.368 \\
2256.063\end{array}$ & $\begin{array}{l}1 \\
3 \\
3 \\
1 \\
3\end{array}$ & $\begin{array}{l}\text { 43963. } 86 \\
43988.68 \\
44026.16 \\
44032.11 \\
44311.28\end{array}$ & $\begin{array}{l}3.91 \\
8.52 \\
6.10 \\
2.40 \\
1.16\end{array}$ & $\begin{array}{l}a^{5} \mathrm{~F}_{3}-u{ }^{5} \mathrm{P}_{3}^{\circ} \\
a^{5} \mathbf{F}_{4}-v{ }^{3} \mathrm{~F}_{3}^{\circ} \\
a^{5} \mathrm{~F}_{1}-t{ }^{3} \mathrm{D}_{1}^{\circ} \\
a^{5} \mathbf{F}_{4}-4_{4}^{\circ} ? \\
a^{5} \mathrm{~F}_{2}-u^{3} \mathrm{D}_{2}^{\circ}\end{array}$ & $\begin{array}{l}\text { UV73 } \\
\text { UV72 } \\
\text { UV75 }\end{array}$ & $\begin{array}{l}\text { New } \\
\text { New } \\
\text { New } \\
\text { New } \\
\text { New }\end{array}$ & $\begin{array}{c}\text { BK, U } \\
\text { BK, X, Z } \\
\text { BK, U } \\
\text { BK, Z } \\
\text { BK }\end{array}$ \\
\hline $\begin{array}{l}2231.090 \\
2222.059 \\
2208.714 \\
2192.819 \\
2190.879\end{array}$ & $\begin{array}{l}2 \\
2 \\
1 \\
3 \\
3\end{array}$ & $\begin{array}{l}44807.22 \\
44989.30 \\
45261.10 \\
45589.15 \\
45629.52\end{array}$ & $\begin{array}{l}7.07 \\
9.14 \\
0.96 \\
9.09 \\
9.46\end{array}$ & $\begin{array}{l}a^{3} \mathrm{~F}_{4}-u{ }^{3} \mathrm{~F}_{3}^{\circ} \\
a^{3} \mathrm{~F}_{3}-t{ }^{3} \mathrm{~F}_{4}^{\circ} \\
a^{5} \mathrm{D}_{2}-x{ }^{5} \mathrm{G}_{2}^{\circ} \\
a^{3} \mathrm{~F}_{4}-57565_{3}^{\circ} ? \\
a^{5} \mathrm{~F}_{3}-53358_{3}^{\circ}\end{array}$ & $\begin{array}{l}\text { UV112 } \\
\text { UV114 } \\
\text { UV20 }\end{array}$ & $\begin{array}{l}\text { New } \\
\text { New } \\
\text { New } \\
\text { New } \\
\text { New }\end{array}$ & $\begin{array}{c}\mathrm{U} \\
\mathrm{BK}, \mathrm{U} \\
\mathrm{BK}, \mathrm{U} \\
\mathrm{BK}, \mathrm{X}, \mathrm{Z} \\
\mathrm{BK}, \mathrm{U}\end{array}$ \\
\hline $\begin{array}{l}2189.720 \\
2185.216 \\
2184.46 \\
2178.797 \\
2177.690\end{array}$ & $\begin{array}{l}1 \\
0 \\
1 \\
2 \\
1\end{array}$ & $\begin{array}{l}45653.66 \\
45747.75 \\
45763.58 \\
45882.52 \\
45905.84\end{array}$ & $\begin{array}{l}\text { 3. } 65 \\
7.71 \\
3.59 \\
2.37 \\
5.74\end{array}$ & $\begin{array}{l}a^{5} \mathrm{~F}_{1}-v{ }^{3} \mathrm{P}_{1}^{\circ} ? \\
a^{5} \mathrm{~F}_{2}-537344^{\circ} \\
a^{5} \mathrm{~F}_{2}-53749_{2}^{\circ} \\
a^{5} \mathrm{~F}_{3}-53610_{4}^{\circ} \\
a^{5} \mathrm{~F}_{2}-10_{3}^{\circ}\end{array}$ & UV80 & $\begin{array}{l}\text { New } \\
\text { New } \\
\text { New } \\
\text { New } \\
\text { New }\end{array}$ & $\begin{array}{c}\text { BK, U } \\
\text { BK, X, Z } \\
Z \text { Z } \\
\text { BK, U } \\
\text { BK, U }\end{array}$ \\
\hline $\begin{array}{l}2174.142 \\
2172.332 \\
2172.221 \\
2170.554 \\
2167.271\end{array}$ & $\begin{array}{l}3 \\
0 \\
1 \\
3 \\
1\end{array}$ & $\begin{array}{l}45980.74 \\
46019.05 \\
46021.40 \\
46056.74 \\
46126.50\end{array}$ & $\begin{array}{l}0.75 \\
9.02 \\
1.32 \\
6.67 \\
6.50\end{array}$ & $\begin{array}{l}a^{5} \mathrm{~F}_{4}-53358_{3}^{\circ} \\
a^{5} \mathrm{~F}_{2}-11_{3}^{\circ} \\
a^{5} \mathrm{~F}_{3}-53749_{2}^{\circ} \\
a^{5} \mathrm{~F}_{3}-53785_{3}^{\circ} \\
a^{5} \mathrm{~F}_{2}-t{ }^{5} \mathrm{P}_{2}^{\circ}\end{array}$ & $\begin{array}{l}\text { UV82 } \\
\text { UV78 }\end{array}$ & $\begin{array}{l}\text { New } \\
\text { New } \\
\text { New } \\
\text { New } \\
\text { New }\end{array}$ & $\begin{array}{l}\text { BK, }, \mathrm{X}, \mathrm{Z} \\
\text { BK } \\
\text { BK } \\
\text { BK, }, \mathrm{X}, \mathrm{Z} \\
\text { BK }\end{array}$ \\
\hline $\begin{array}{l}2165.982 \\
2162.243 \\
2158.993 \\
2158.732 \\
2156.504\end{array}$ & $\begin{array}{l}3 \\
3 \\
1 \\
3 \\
3\end{array}$ & $\begin{array}{l}46153.95 \\
46233.75 \\
46303.34 \\
46308.94 \\
46356.78\end{array}$ & $\begin{array}{l}\text { 3. } 84 \\
\text { 3. } 66 \\
\text { 3. } 29 \\
\text { 8. } 94 \\
\text { 6. } 73\end{array}$ & $\begin{array}{l}a^{5} \mathrm{~F}_{3}-53882_{4}^{\circ} \\
a^{5} \mathrm{~F}_{4}-53610_{4}^{\circ} \\
a^{5} \mathrm{~F}_{2}-54289^{\circ} \\
a^{5} \mathrm{D}_{1}-x{ }^{3} \mathrm{~F}_{2}^{\circ} \\
a^{5} \mathrm{~F}_{4}-53734^{2} ?\end{array}$ & UV 25 & $\begin{array}{l}\text { New } \\
\text { New } \\
\text { New } \\
\text { New } \\
\text { New d }\end{array}$ & $\begin{array}{c}\text { BK } \\
\text { BK, X, Z } \\
\text { BK } \\
\text { BK } \\
\text { BK, X, Z }\end{array}$ \\
\hline $\begin{array}{l}2155.816 \\
2155.114 \\
2154.127 \\
2149.620 \\
2147.039\end{array}$ & $\begin{array}{l}3 \\
0 \\
3 \\
1 \\
3\end{array}$ & $\begin{array}{l}46371.57 \\
46386.67 \\
46407.92 \\
46505.22 \\
46561.12\end{array}$ & $\begin{array}{l}\text { 1. } 50 \\
6.50 \\
\text { 7. } 96 \\
\text { 5. } 13 \\
\text { 1. } 02\end{array}$ & $\begin{array}{l}a^{5} \mathrm{~F}_{2}-54357^{\circ} \\
a^{5} \mathrm{~F}_{4}-x{ }^{1} \mathrm{~F}_{3}^{\circ} \\
a^{5} \mathrm{~F}_{4}-53785_{3}^{\circ} \\
a^{5} \mathrm{~F}_{4}-53882_{4}^{\circ} \\
a^{5} \mathrm{~F}_{3}-54289_{3}^{\circ}\end{array}$ & & $\begin{array}{l}\text { New } \\
\text { New } \\
\text { New } \\
\text { New } \\
\text { New }\end{array}$ & $\begin{array}{c}\text { BK, X, Z } \\
\text { BK } \\
\text { U } \\
\text { BK, X, Z } \\
\text { BK, X, Z }\end{array}$ \\
\hline $\begin{array}{l}2143.892 \\
2141.471 \\
2130.962 \\
2127.863 \\
2127.467\end{array}$ & $\begin{array}{l}3 \\
3 \\
3 \\
2 \\
1\end{array}$ & $\begin{array}{l}46629.45 \\
46682.16 \\
46912.35 \\
46980.67 \\
46989.41\end{array}$ & $\begin{array}{l}9.33 \\
2.16 \\
2.31 \\
0.62 \\
9.29\end{array}$ & $\begin{array}{l}a^{5} \mathrm{~F}_{3}-54357_{3} \\
a^{5} \mathrm{~F}_{5}-53610_{4}^{\circ} \\
a^{5} \mathrm{~F}_{4}-54289_{3}^{\circ} \\
a^{5} \mathrm{~F}_{4}-54357^{\circ} \\
a^{5} \mathrm{D}_{2}-w^{5} \mathrm{G}_{3}^{\circ}\end{array}$ & UV28 & $\begin{array}{l}\text { New } \\
\text { New } \\
\text { New } \\
\text { New } \\
\text { New }\end{array}$ & $\begin{array}{c}\text { BK, Z } \\
\text { Z } \\
\text { C } \\
\text { BK, X, Z } \\
\text { BK, U }\end{array}$ \\
\hline $\begin{array}{l}\text { 2124. } 494 \\
2123.118 \\
2111.220 \\
\text { (vac.) }\end{array}$ & $\begin{array}{l}0 \\
0 \\
0\end{array}$ & $\begin{array}{l}47055.16 \\
47085.65 \\
47350.98\end{array}$ & $\begin{array}{l}5.02 \\
5.50 \\
0.77\end{array}$ & $\begin{array}{l}a^{5} \mathrm{~F}_{5}-t{ }^{3} \mathrm{G}_{5}^{\circ} \\
a^{5} \mathrm{~F}_{5}-12_{5}^{\circ} \\
a^{5} \mathrm{D}_{1}-v{ }^{5} \mathrm{~F}_{2}^{\circ}\end{array}$ & $\begin{array}{l}\text { UV81 } \\
\text { UV31 }\end{array}$ & $\begin{array}{l}\text { New } \\
\text { New } \\
\text { New }\end{array}$ & $\begin{array}{c}\mathrm{BK}, \mathrm{U} \\
\mathrm{BK}, \mathrm{U} \\
\mathrm{BK}, \mathrm{N}, \mathrm{U}\end{array}$ \\
\hline $\begin{array}{l}\text { 1899. } 21 \\
1891.74 \\
1883.91 \\
1865.30 \\
1859.26\end{array}$ & $\begin{array}{r}1 \\
10 \\
2 \\
15 \\
2\end{array}$ & $\begin{array}{l}52653.4 \\
52861.5 \\
53081.2 \\
53610.6 \\
53784.9\end{array}$ & $\begin{array}{l}\text { 3. } 53 \\
\text { 1. } 26 \\
0.74 \\
0.44 \\
\text { 4. } 79\end{array}$ & $\begin{array}{l}a^{5} \mathrm{D}_{2}-53358_{3}^{\circ} \\
a^{5} \mathrm{D}_{1}-53749_{2}^{\circ} \\
a^{5} \mathrm{D}_{2}-53785_{3}^{\circ} \\
a^{5} \mathrm{D}_{4}-53610_{4}^{\circ} \\
a^{5} \mathrm{D}_{4}-53785_{3}^{\circ}\end{array}$ & & $\begin{array}{l}\text { New } \\
\text { New } \\
\text { New } \\
\text { New } \\
\text { New }\end{array}$ & $\begin{array}{l}\mathrm{N} \\
\mathrm{N} \\
\mathrm{N} \\
\mathrm{N} \\
\mathrm{N}\end{array}$ \\
\hline
\end{tabular}

a Improved wavelength of previously known line listed here as preferable to the value quoted in Table $B$ of the Monograph.[1]

b Predicted line not listed in Table $\mathrm{C}$ of the Monograph. See Ref. 3

c Blend of Fe I and Fe II. See J. C. Dobbie, Ann. Solar Phys, Obs. Cambridge, $\mathbf{V}$, pt. 1,1938 . d Member of resolved doublet.

e Designation in Table B of the Monograph to be rejected.

$i$ This line is listed as $10 \mathrm{r}$ III by A. S. King; the line classified here may be masked. 


\section{Reference Sources for Wavelengths}

BK K. Burns and C. C. Kiess, unpublished material (1934).

C W. F. Meggers and C. J. Humphreys, NBS J. Research 18, 543, RP 992 (1937).

D C. C. Kiess, NBS J. Research 20, 33, RP 1062 (1938).

G K. Burns and F. M. Walters, Jr., Publ. Allegheny Obs. 8, 39, No. 4 (1931).

J C. E. St. John and H. D. Babcock, Mt. Wilson Contr. No. 202; Astroph. J. 53, 260 (1921) (corrected).

N L. C. Green, Phys. Rev. 55, 1209 (1939), and unpublished material (1937).

O W. F. Meggers and C.C. Kiess, BS Sci. Pap. 14, 642, No. 324 (1918).
S J. C. Dobbie, unpublished material.

$\mathrm{T}$ Sinclair Smith, unpublished material.

U G. R. Harrison, unpublished material, Mass. Inst. Tech. (June 1942).

V K. Burns, Lick Obs. Bull. 8, 27, No. 247 (1913).

W H. Kayser, Handbuch der Spectroscopie 6, 896 (1912).

X H. Schumacher, Zeit. Wiss. Ptg. 19, 149 (1919) (corrected).

Z G. R. Harrison, Mass. Inst. Tech. Wavelength Tables (John Wiley and Sons, Inc., New York, 1956).

ZZ H. M. Crosswhite, Johns Hopkins Spectroscopic Report No. 13, 120 pp. (1958).

TABLE 4.-New Fe I lines, unclassified

\begin{tabular}{|c|c|c|c|c|c|c|c|}
\hline $\begin{array}{l}\text { Wavelength } \\
\text { A }\end{array}$ & Intensity & $\begin{array}{l}\text { Wave Number } \\
\mathrm{cm}^{-1}\end{array}$ & $\begin{array}{l}\text { Notes and } \\
\text { References }\end{array}$ & $\begin{array}{c}\text { Wavelength } \\
\text { A }\end{array}$ & Intensity & $\begin{array}{l}\text { Wave Number } \\
\mathrm{cm}^{-1}\end{array}$ & $\begin{array}{l}\text { Notes and } \\
\text { References }\end{array}$ \\
\hline $\begin{array}{l}8558.647 \\
7952.338 \\
7945.090 \\
7937.914 \\
7854.150\end{array}$ & $\begin{array}{l}1 \\
1 \\
2 \\
1 n \\
0 n\end{array}$ & $\begin{array}{l}11680.88 \\
12571.46 \\
12582.93 \\
12594.30 \\
12728.62\end{array}$ & & $\begin{array}{l}6347.162 \\
6333.354 \\
6242.738 \\
6143.052 \\
5884.323\end{array}$ & $\begin{array}{l}3 \\
3 \\
3 \\
3 \\
1\end{array}$ & $\begin{array}{l}15750.72 \\
15785.06 \\
16014.18 \\
16274.05 \\
16989.60\end{array}$ & \\
\hline $\begin{array}{l}7775.383 \\
7714.603 \\
7702.968 \\
7693.734 \\
7675.972\end{array}$ & $\begin{array}{l}1 \\
0 \\
0 \\
0 \\
1\end{array}$ & $\begin{array}{l}12857.56 \\
12958.86 \\
12978.44 \\
12994.01 \\
13024.08\end{array}$ & & $\begin{array}{l}5699.308 \\
5644.033 \\
5625.704 \\
5601.298 \\
5590.661\end{array}$ & $\begin{array}{l}2 \\
3 \\
3 \\
2 \\
3\end{array}$ & $\begin{array}{l}17541.12 \\
17712.91 \\
17770.62 \\
17848.05 \\
17882.01\end{array}$ & \\
\hline $\begin{array}{l}7674.966 \\
7657.254 \\
7643.394 \\
7626.473 \\
7624.011\end{array}$ & $\begin{array}{l}0 \\
0 \\
0 \\
0 \\
0\end{array}$ & $\begin{array}{l}13025.79 \\
13055.92 \\
13079.59 \\
13108.61 \\
13112.85\end{array}$ & & $\begin{array}{l}5589.852 \\
5582.673 \\
5553.176 \\
5532.856 \\
5521.096\end{array}$ & $\begin{array}{l}2 n \\
2 n \\
2 n \\
2 \\
2\end{array}$ & $\begin{array}{l}17884.60 \\
17907.59 \\
18002.71 \\
18068.83 \\
18107.32\end{array}$ & $\mathrm{U}, \mathrm{W}$ \\
\hline $\begin{array}{l}7615.529 \\
7614.148 \\
7600.948 \\
7599.624 \\
7596.842\end{array}$ & $\begin{array}{l}0 \\
0 \\
0 \\
0 \\
0\end{array}$ & $\begin{array}{l}13127.45 \\
13129.83 \\
13152.63 \\
13154.92 \\
13159.74\end{array}$ & & $\begin{array}{l}5512.658 \\
5477.744 \\
5458.572 \\
5418.598 \\
5351.751\end{array}$ & $\begin{array}{l}3 n \\
1 n \\
2 \\
2 n \\
1\end{array}$ & $\begin{array}{l}18135.03 \\
18250.6 \\
18314.72 \\
18449.83 \\
18680.28\end{array}$ & W \\
\hline $\begin{array}{l}7588.834 \\
7580.647 \\
7576.505 \\
7573.444 \\
7567.043\end{array}$ & $\begin{array}{l}0 \\
0 \\
0 \\
0 \\
0\end{array}$ & $\begin{array}{l}13173.63 \\
13187.86 \\
13195.06 \\
13200.40 \\
13211.56\end{array}$ & $\mathrm{U}$ & $\begin{array}{l}5350.798 \\
5350.434 \\
5333.396 \\
5319.079 \\
5317.854\end{array}$ & $\begin{array}{l}1 \\
0 \\
2 \\
2 n \\
1\end{array}$ & $\begin{array}{l}18683.60 \\
18684.88 \\
18744.57 \\
18795.02 \\
18799.35\end{array}$ & \\
\hline $\begin{array}{l}7554.743 \\
7528.735 \\
7523.388 \\
7501.061 \\
7476.098\end{array}$ & $\begin{array}{l}0 \\
0 \\
0 \\
0 \\
0\end{array}$ & $\begin{array}{l}13233.07 \\
13278.79 \\
13288.22 \\
13327.78 \\
13372.28\end{array}$ & & $\begin{array}{l}5313.542 \\
5312.539 \\
5312.024 \\
5311.641 \\
5310.855\end{array}$ & $\begin{array}{l}1 n \\
0 \\
0 \\
1 \\
0 n\end{array}$ & $\begin{array}{l}18814.60 \\
18818.16 \\
18819.98 \\
18821.34 \\
18824.12\end{array}$ & \\
\hline $\begin{array}{l}7453.230 \\
7438.336 \\
7435.798 \\
7214.630 \\
7192.458\end{array}$ & $\begin{array}{l}1 \\
1 \\
0 n \\
1 \\
1 n\end{array}$ & $\begin{array}{l}\text { 13413. } 31 \\
13440.16 \\
13444.75 \\
13856.91 \\
13899.62\end{array}$ & & $\begin{array}{l}\text { 5310. } 270 \\
\text { 5309. } 780 \\
\text { 5308. } 228 \\
\text { 5307. } 736 \\
\text { 5306. } 060\end{array}$ & $\begin{array}{l}0 n \\
0 \\
0 \\
1 \\
0 n\end{array}$ & $\begin{array}{l}\text { 18826. } 20 \\
18827.93 \\
18833.44 \\
18835.18 \\
18841.13\end{array}$ & $\mathrm{U}, \mathrm{W}$ \\
\hline $\begin{array}{l}7009.530 \\
6864.229 \\
6692.280 \\
6587.388 \\
6515.104\end{array}$ & $\begin{array}{l}1 \\
1 \\
1 \\
1 \\
1 n\end{array}$ & $\begin{array}{l}14262.36 \\
14564.26 \\
14938.47 \\
15176.33 \\
15344.71\end{array}$ & & $\begin{array}{l}5303.874 \\
5301.408 \\
5199.090 \\
5182.983 \\
5181.335\end{array}$ & $\begin{array}{l}1 n \\
1 \\
3 \\
2 \\
3\end{array}$ & $\begin{array}{l}\text { 18848. } 90 \\
18857.67 \\
19228.78 \\
19288.54 \\
19294.67\end{array}$ & $\mathrm{~T}, \mathrm{U}$ \\
\hline $\begin{array}{l}6491.395 \\
6478.828 \\
6452.148 \\
6392.988 \\
6366.040\end{array}$ & $\begin{array}{l}1 \\
1 \\
1 n \\
3 \\
3\end{array}$ & $\begin{array}{l}15400.75 \\
15430.63 \\
15494.43 \\
15637.82 \\
15704.01\end{array}$ & & $\begin{array}{l}5176.806 \\
5173.498 \\
5166.694 \\
5160.094 \\
5154.039\end{array}$ & $\begin{array}{l}1 \\
3 \\
3 \\
3 n \\
2 n\end{array}$ & $\begin{array}{l}19311.55 \\
19323.90 \\
19349.35 \\
19374.10 \\
19396.86\end{array}$ & \\
\hline
\end{tabular}


Table 4.-New Fe I lines, unclassified-Continued

\begin{tabular}{|c|c|c|c|c|c|c|c|}
\hline $\begin{array}{c}\text { Wavelength } \\
\text { A }\end{array}$ & Intensity & $\begin{array}{l}\text { Wave Number } \\
\mathrm{cm}^{-1}\end{array}$ & $\begin{array}{l}\text { Notes and } \\
\text { References }\end{array}$ & $\begin{array}{c}\text { Wavelength } \\
\text { A }\end{array}$ & Intensity & $\begin{array}{l}\text { Wave Number } \\
\mathrm{cm}^{-1}\end{array}$ & $\begin{array}{l}\text { Notes and } \\
\text { References }\end{array}$ \\
\hline $\begin{array}{l}5149.746 \\
5147.107 \\
5140.826 \\
5097.498 \\
5025.514\end{array}$ & $\begin{array}{l}3 \\
2 \\
3 \\
3 \\
3\end{array}$ & $\begin{array}{l}19413.02 \\
19422.98 \\
19446.71 \\
19612.00 \\
19892.91\end{array}$ & & $\begin{array}{l}4712.515 \\
4710.650 \\
4703.228 \\
4702.642 \\
4702.299\end{array}$ & $\begin{array}{l}1 \\
0 \\
0 \\
0 \\
0 n\end{array}$ & $\begin{array}{l}21214.16 \\
21222.56 \\
21256.04 \\
21258.69 \\
21260.24\end{array}$ & \\
\hline $\begin{array}{l}\text { 5013. } 914 \\
\text { 4996. } 792 \\
\text { 4996. } 174 \\
4992.502 \\
\text { 4974. } 246\end{array}$ & $\begin{array}{l}3 \\
3 \\
0 n \\
1 \\
0\end{array}$ & $\begin{array}{l}\text { 19938. } 94 \\
20007.26 \\
20009.73 \\
20024.45 \\
20097.94\end{array}$ & $\begin{array}{c}\mathrm{U} \\
\mathrm{U}, \mathrm{W}\end{array}$ & $\begin{array}{l}4701.849 \\
4699.424 \\
4691.769 \\
4688.964 \\
4686.641\end{array}$ & $\begin{array}{l}0 n \\
3 n \\
0 \\
0 \\
0\end{array}$ & $\begin{array}{l}21262.28 \\
21273.25 \\
21307.96 \\
21320.71 \\
21331.27\end{array}$ & $\mathrm{BK}$ \\
\hline $\begin{array}{l}4961.040 \\
4948.299 \\
4947.645 \\
4947.418 \\
4944.306\end{array}$ & $\begin{array}{l}3 \\
1 \\
3 n \\
0 \\
3\end{array}$ & $\begin{array}{l}\text { 20151. } 44 \\
\text { 20203. } 33 \\
20206.00 \\
\text { 20206. } 92 \\
\text { 20219. } 64\end{array}$ & $\begin{array}{l}\mathrm{U}, \mathrm{W} \\
\mathrm{U}\end{array}$ & $\begin{array}{l}4686.348 \\
4685.583 \\
4684.662 \\
4680.628 \\
4676.757\end{array}$ & $\begin{array}{l}0 n \\
0 n \\
2 \\
1 \\
0\end{array}$ & $\begin{array}{l}21332.61 \\
21336.09 \\
21340.28 \\
21358.68 \\
21376.36\end{array}$ & \\
\hline $\begin{array}{l}\text { 4932. } 134 \\
4924.333 \\
4915.806 \\
4913.168 \\
4911.587\end{array}$ & $\begin{array}{l}3 \\
3 \\
3 \\
3 \\
3\end{array}$ & $\begin{array}{l}20269.54 \\
20301.65 \\
20336.87 \\
20347.78 \\
20354.34\end{array}$ & $\mathrm{U}$ & $\begin{array}{l}4674.297 \\
4671.337 \\
4664.750 \\
4660.920 \\
4660.478\end{array}$ & $\begin{array}{l}2 \\
1 n \\
1 \\
1 n \\
1 n\end{array}$ & $\begin{array}{l}21387.60 \\
21401.16 \\
21431.38 \\
21448.99 \\
21451.02\end{array}$ & $\mathrm{U}, \mathrm{W}$ \\
\hline $\begin{array}{l}\text { 4906. } 144 \\
4902.368 \\
4901.272 \\
4900.816 \\
4900.520\end{array}$ & $\begin{array}{l}0 \\
1 \\
2 \\
2 \\
0\end{array}$ & $\begin{array}{l}\text { 20376. } 92 \\
20392.61 \\
20397.17 \\
20399.07 \\
20400.30\end{array}$ & & $\begin{array}{l}4656.548 \\
4650.388 \\
4648.986 \\
4640.958 \\
4640.340\end{array}$ & $\begin{array}{l}1 n \\
0 n \\
0 \\
1 \\
2 n\end{array}$ & $\begin{array}{l}21469.12 \\
21497.56 \\
21504.05 \\
21541.24 \\
21544.11\end{array}$ & $\begin{array}{l}\left.{ }^{(}\right) \\
\mathrm{U}, \mathrm{W} \\
\mathrm{U}, \mathrm{W}\end{array}$ \\
\hline $\begin{array}{l}\text { 4898. } 930 \\
4895.672 \\
4880.976 \\
4875.081 \\
4864.512\end{array}$ & $\begin{array}{l}0 \\
0 \\
2 \\
3 \\
2\end{array}$ & $\begin{array}{l}20406.92 \\
20420.50 \\
20482.82 \\
20506.75 \\
20551.31\end{array}$ & & $\begin{array}{l}4605.610 \\
4597.403 \\
4590.815 \\
4585.337 \\
4582.297\end{array}$ & $\begin{array}{l}2 n \\
2 \\
2 n \\
1 \\
1 n\end{array}$ & $\begin{array}{l}21706.57 \\
21745.32 \\
21776.52 \\
21802.54 \\
21817.00\end{array}$ & $\begin{array}{c}\mathrm{BK}, \mathrm{U} \\
\mathrm{U}, \mathrm{W}\end{array}$ \\
\hline $\begin{array}{l}4862.992 \\
4861.947 \\
4833.817 \\
4832.036 \\
4825.357\end{array}$ & $\begin{array}{l}3 \\
3 \\
1 \\
1 \\
3\end{array}$ & $\begin{array}{l}\text { 20557. } 73 \\
\text { 20562. } 15 \\
20681.81 \\
20689.43 \\
20718.07\end{array}$ & $\begin{array}{l}\mathrm{U}, \mathrm{W} \\
\mathrm{U}, \mathrm{W}\end{array}$ & $\begin{array}{l}4581.186 \\
4566.940 \\
4560.892 \\
4557.287 \\
4533.078\end{array}$ & $\begin{array}{l}1 \\
3 \\
1 \\
2 \\
2 n\end{array}$ & $\begin{array}{l}21822.29 \\
21890.36 \\
21919.39 \\
21936.73 \\
22053.88\end{array}$ & $\begin{array}{c}\mathrm{U}, \mathrm{W} \\
\mathrm{U} \\
\mathrm{U}\end{array}$ \\
\hline $\begin{array}{l}\text { 4821. } 572 \\
4818.038 \\
4814.365 \\
4811.407 \\
4805.072\end{array}$ & $\begin{array}{l}2 \\
1 \\
2 \\
0 \\
2 n\end{array}$ & $\begin{array}{l}20734.33 \\
20749.54 \\
20765.37 \\
20778.13 \\
20805.53\end{array}$ & & $\begin{array}{l}4517.136 \\
4509.804 \\
4509.430 \\
4500.652 \\
4480.731\end{array}$ & $\begin{array}{l}1 \\
2 n \\
2 \\
1 n \\
1 n\end{array}$ & $\begin{array}{l}22131.71 \\
22167.70 \\
22169.53 \\
22212.77 \\
22311.53\end{array}$ & $\begin{array}{c}\mathrm{U}, \mathrm{W} \\
\left(^{\mathrm{c}}\right)\end{array}$ \\
\hline $\begin{array}{l}4793.336 \\
4784.034 \\
4782.147 \\
4776.451 \\
4774.939\end{array}$ & $\begin{array}{l}0 \\
2 \\
1 n \\
3 n \\
1 n\end{array}$ & $\begin{array}{l}20856.47 \\
20897.02 \\
20905.26 \\
20930.19 \\
20936.82\end{array}$ & & $\begin{array}{l}4478.649 \\
4471.600 \\
4468.452 \\
4465.552 \\
4460.642\end{array}$ & $\begin{array}{l}1 n \\
0 \\
0 \\
0 \\
0\end{array}$ & $\begin{array}{l}22321.90 \\
22357.09 \\
22372.84 \\
22387.36 \\
22412.01\end{array}$ & \\
\hline $\begin{array}{l}4768.697 \\
4766.821 \\
4756.888 \\
4756.356 \\
4752.470\end{array}$ & $\begin{array}{l}3 \\
3 \\
0 \\
1 \\
1\end{array}$ & $\begin{array}{l}20964.23 \\
20972.48 \\
21016.27 \\
21018.62 \\
21035.81\end{array}$ & $\mathrm{U}$ & $\begin{array}{l}4452.773 \\
4451.684 \\
4445.050 \\
4432.087 \\
4431.799\end{array}$ & $\begin{array}{l}1 n \\
0 n \\
0 \\
1 n \\
1 n\end{array}$ & $\begin{array}{l}22451.61 \\
22457.11 \\
22490.62 \\
22556.40 \\
22557.87\end{array}$ & $\begin{array}{l}\mathrm{W} \\
\mathrm{U}\end{array}$ \\
\hline $\begin{array}{l}4746.278 \\
4733.945 \\
4730.009 \\
4728.160 \\
4722.522\end{array}$ & $\begin{array}{l}0 \\
0 \\
3 n \\
1 \\
0 n\end{array}$ & $\begin{array}{l}21063.25 \\
21118.12 \\
21135.70 \\
21143.96 \\
21169.20\end{array}$ & & $\begin{array}{l}4425.090 \\
4424.608 \\
4424.061 \\
4420.266 \\
4416.688\end{array}$ & $\begin{array}{l}0 n \\
1 \\
1 \\
2 n \\
0\end{array}$ & $\begin{array}{l}22592.07 \\
22594.53 \\
22597.32 \\
22616.72 \\
22635.04\end{array}$ & $\mathrm{~W}$ \\
\hline $\begin{array}{l}\text { 4719. } 716 \\
4719.238 \\
4717.620 \\
4717.358 \\
4716.483\end{array}$ & $\begin{array}{l}0 \\
0 n \\
2 n \\
2 n \\
0\end{array}$ & $\begin{array}{l}\text { 21181. } 79 \\
21183.94 \\
21191.20 \\
21192.38 \\
21196.31\end{array}$ & & $\begin{array}{l}4416.423 \\
4416.137 \\
4412.146 \\
4411.914 \\
4406.946\end{array}$ & $\begin{array}{l}1 \\
0 \\
1 \\
2 n \\
1\end{array}$ & $\begin{array}{l}22636.40 \\
22637.87 \\
22658.34 \\
22659.54 \\
22685.08\end{array}$ & $\mathrm{U}$ \\
\hline
\end{tabular}


Taвle 4.-New Fe I lines, unclassified-Continued

\begin{tabular}{|c|c|c|c|c|c|c|c|}
\hline $\begin{array}{c}\text { Wavelength } \\
\mathrm{A}\end{array}$ & Intensity & $\begin{array}{l}\text { Wave Number } \\
\text { cm }^{-1}\end{array}$ & $\begin{array}{l}\text { Notes and } \\
\text { References }\end{array}$ & $\begin{array}{c}\text { Wavelength } \\
\mathrm{A}\end{array}$ & Intensity & $\begin{array}{l}\text { Wave Number } \\
\mathrm{cm}^{-1}\end{array}$ & $\begin{array}{l}\text { Notes and } \\
\text { References }\end{array}$ \\
\hline $\begin{array}{l}\text { 4399. } 260 \\
\text { 4395. } 937 \\
\text { 4394. } 098 \\
\text { 4385. } 776 \\
\text { 4384. } 848\end{array}$ & $\begin{array}{l}0 \\
0 n \\
1 \\
1 n \\
2\end{array}$ & $\begin{array}{l}22724.71 \\
22741.89 \\
22751.41 \\
22794.58 \\
22799.40\end{array}$ & $\mathrm{U}$ & $\begin{array}{l}\text { 4282. } 052 \\
4281.921 \\
4281.855 \\
4281.490 \\
4280.285\end{array}$ & $\begin{array}{l}1 n \\
1 n \\
1 \\
1 \\
1 n\end{array}$ & $\begin{array}{l}\text { 23346. } 72 \\
23347.43 \\
23347.79 \\
23349.78 \\
23356.36\end{array}$ & $\underset{\mathrm{U}}{\mathrm{BK}}$ \\
\hline $\begin{array}{l}\text { 4381. } 429 \\
4380.108 \\
4378.486 \\
4376.446 \\
4374.988\end{array}$ & $\begin{array}{l}1 n \\
1 \\
2 \\
1 \\
1\end{array}$ & $\begin{array}{l}22817.19 \\
22824.07 \\
22832.53 \\
22843.17 \\
22850.78\end{array}$ & & $\begin{array}{l}4278.604 \\
4276.082 \\
4272.528 \\
4270.838 \\
4270.628\end{array}$ & $\begin{array}{l}1 n \\
1 \\
1 \\
1 \\
1 n\end{array}$ & $\begin{array}{l}23365.53 \\
23379.32 \\
23398.76 \\
23408.02 \\
23409.17\end{array}$ & $\mathrm{U}$ \\
\hline $\begin{array}{l}\text { 4374. } 116 \\
4372.558 \\
4371.742 \\
4364.772 \\
4362.484\end{array}$ & $\begin{array}{l}1 \\
1 \\
0 \\
1 \\
1\end{array}$ & $\begin{array}{l}22855.34 \\
22863.48 \\
22867.75 \\
22904.27 \\
22916.28\end{array}$ & & $\begin{array}{l}4270.251 \\
4269.730 \\
4252.911 \\
4251.999 \\
4251.657\end{array}$ & $\begin{array}{l}1 n \\
1 n \\
1 n \\
1 n \\
0 n\end{array}$ & $\begin{array}{l}23411.24 \\
23414.10 \\
23506.69 \\
23511.73 \\
23513.62\end{array}$ & $\mathrm{U}$ \\
\hline $\begin{array}{l}\text { 4357. } 940 \\
4357.098 \\
4355.144 \\
4347.000 \\
4344.928\end{array}$ & $\begin{array}{l}0 \\
0 \\
1 \\
0 \\
1\end{array}$ & $\begin{array}{l}22940.17 \\
22944.61 \\
22954.90 \\
22997.91 \\
23008.87\end{array}$ & $\mathrm{U}$ & $\begin{array}{l}4251.288 \\
4249.596 \\
4249.216 \\
4236.461 \\
4234.122\end{array}$ & $\begin{array}{l}1 n \\
1 n \\
1 \\
1 \\
1 n\end{array}$ & $\begin{array}{l}23515.66 \\
23525.03 \\
23527.13 \\
23597.96 \\
23611.00\end{array}$ & $\mathrm{U}$ \\
\hline $\begin{array}{l}\text { 4341. } 392 \\
4340.950 \\
4340.279 \\
4337.455 \\
4337.366\end{array}$ & $\begin{array}{l}1 \\
0 \\
0 \\
0 \\
1\end{array}$ & $\begin{array}{l}23027.61 \\
23029.96 \\
23033.52 \\
23048.51 \\
23048.99\end{array}$ & & $\begin{array}{l}4229.406 \\
4228.384 \\
4223.221 \\
4219.850 \\
4219.018\end{array}$ & $\begin{array}{l}1 \\
1 n \\
1 \\
1 \\
1\end{array}$ & $\begin{array}{l}23637.32 \\
23643.04 \\
23671.94 \\
23690.85 \\
23695.52\end{array}$ & $\begin{array}{c}\mathrm{U} \\
\mathrm{U} \\
\mathrm{W}\end{array}$ \\
\hline $\begin{array}{l}\text { 4336. } 122 \\
\text { 4335. } 773 \\
\text { 4335. } 399 \\
\text { 4334. } 928 \\
4334.428\end{array}$ & $\begin{array}{l}1 \\
0 \\
0 \\
1 n \\
0 n\end{array}$ & $\begin{array}{l}23055.60 \\
23057.46 \\
23059.44 \\
23061.95 \\
23064.61\end{array}$ & $\mathrm{U}, \mathrm{W}$ & $\begin{array}{l}4218.880 \\
4218.812 \\
4218.464 \\
4211.568 \\
4210.868\end{array}$ & $\begin{array}{l}1 \\
1 \\
1 \\
1 \\
1\end{array}$ & $\begin{array}{l}23696.30 \\
23696.68 \\
23698.64 \\
23737.44 \\
23741.38\end{array}$ & \\
\hline $\begin{array}{l}\text { 4332. } 711 \\
4332.432 \\
4331.411 \\
4330.640 \\
4327.410\end{array}$ & $\begin{array}{l}1 \\
1 \\
1 n \\
1 \\
0\end{array}$ & $\begin{array}{l}\text { 23073. } 75 \\
23075.24 \\
23080.68 \\
23084.78 \\
23102.01\end{array}$ & $\begin{array}{l}\mathrm{U} \\
\mathrm{U} \\
\mathrm{U}\end{array}$ & $\begin{array}{l}4209.447 \\
4206.210 \\
4203.258 \\
4199.557 \\
4197.512\end{array}$ & $\begin{array}{l}1 \\
1 n \\
1 n \\
1 n \\
0\end{array}$ & $\begin{array}{l}23749.40 \\
23767.68 \\
23784.37 \\
23805.33 \\
23816.93\end{array}$ & $\begin{array}{l}\mathrm{U} \\
\mathrm{U}\end{array}$ \\
\hline $\begin{array}{l}4323.658 \\
4320.767 \\
4319.231 \\
4316.832 \\
4316.525\end{array}$ & $\begin{array}{l}1 n \\
1 n \\
0 \\
0 \\
0\end{array}$ & $\begin{array}{l}23122.06 \\
23137.53 \\
23145.76 \\
23158.62 \\
23160.27\end{array}$ & $\mathrm{U}$ & $\begin{array}{l}\text { 4194. } 099 \\
4193.600 \\
4192.794 \\
4192.622 \\
4192.374\end{array}$ & $\begin{array}{l}1 \\
1 \\
0 \\
1 n \\
1 n\end{array}$ & $\begin{array}{l}\text { 23836. } 31 \\
23839.14 \\
23843.72 \\
23844.70 \\
23846.11\end{array}$ & $\mathrm{U}, \mathrm{W}$ \\
\hline $\begin{array}{l}4315.495 \\
4315.400 \\
4313.812 \\
4312.950 \\
4310.531\end{array}$ & $\begin{array}{l}0 \\
0 \\
1 \\
1 n \\
1\end{array}$ & $\begin{array}{l}23165.80 \\
23166.31 \\
23174.84 \\
23179.47 \\
23192.48\end{array}$ & $\begin{array}{l}\mathrm{W} \\
\mathrm{U}\end{array}$ & $\begin{array}{l}4191.974 \\
4190.938 \\
4190.000 \\
4189.015 \\
4188.300\end{array}$ & $\begin{array}{l}1 n \\
1 n \\
1 n \\
1 n \\
1 n\end{array}$ & $\begin{array}{l}23848.39 \\
23854.28 \\
23859.62 \\
23865.24 \\
23869.31\end{array}$ & \\
\hline $\begin{array}{l}4309.704 \\
4306.991 \\
4306.068 \\
4302.541 \\
4302.344\end{array}$ & $\begin{array}{l}0 \\
1 \\
0 \\
0 \\
0\end{array}$ & $\begin{array}{l}23196.92 \\
23211.54 \\
23216.51 \\
23235.54 \\
23236.61\end{array}$ & BK & $\begin{array}{l}4185.793 \\
4184.546 \\
4184.400 \\
4181.278 \\
4189.176\end{array}$ & $\begin{array}{l}3 \\
1 \\
1 \\
1 n \\
0\end{array}$ & $\begin{array}{l}23883.60 \\
23890.72 \\
23891.56 \\
23909.39 \\
23915.70\end{array}$ & $\mathrm{U}$ \\
\hline $\begin{array}{l}\text { 4299. } 770 \\
4298.324 \\
4297.106 \\
4293.612 \\
4293.400\end{array}$ & $\begin{array}{l}1 \\
1 \\
1 n \\
1 n \\
0 n\end{array}$ & $\begin{array}{l}23250.52 \\
23258.34 \\
23264.93 \\
23283.86 \\
23285.01\end{array}$ & $\begin{array}{l}\mathrm{U} \\
\mathrm{U}\end{array}$ & $\begin{array}{l}4179.689 \\
4174.208 \\
4160.333 \\
4158.366 \\
4157.306\end{array}$ & $\begin{array}{l}1 \\
0 n \\
1 n \\
1 \\
1\end{array}$ & $\begin{array}{l}23918.48 \\
23949.89 \\
24029.76 \\
24041.13 \\
24047.26\end{array}$ & $\begin{array}{l}\mathrm{U} \\
\mathrm{U}\end{array}$ \\
\hline $\begin{array}{l}\text { 4288. } 297 \\
4287.952 \\
4285.984 \\
4283.770 \\
4282.914\end{array}$ & $\begin{array}{l}1 \\
1 \\
1 \\
1 \\
1 n\end{array}$ & $\begin{array}{l}23312.72 \\
23314.60 \\
23325.30 \\
23337.36 \\
23342.02\end{array}$ & BK & $\begin{array}{l}\text { 4156. } 322 \\
4155.914 \\
4152.778 \\
4152.651 \\
4148.794\end{array}$ & $\begin{array}{l}1 n \\
1 \\
1 \\
1 \\
1 n\end{array}$ & $\begin{array}{l}24052.95 \\
24055.31 \\
24073.48 \\
24074.21 \\
24096.60\end{array}$ & $\begin{array}{l}\mathrm{U} \\
\mathrm{U}\end{array}$ \\
\hline
\end{tabular}


TABLE 4.-New Fe I lines, unclassified-Continued

\begin{tabular}{|c|c|c|c|c|c|c|c|}
\hline $\begin{array}{c}\text { Wavelength } \\
\text { A }\end{array}$ & Intensity & $\begin{array}{l}\text { Wave Number } \\
\mathrm{cm}^{-1}\end{array}$ & $\begin{array}{l}\text { Notes and } \\
\text { References }\end{array}$ & $\begin{array}{c}\text { Wavelength } \\
\text { A }\end{array}$ & Intensity & $\begin{array}{l}\text { Wave Number } \\
\mathrm{cm}^{-1}\end{array}$ & $\begin{array}{l}\text { Notes and } \\
\text { References }\end{array}$ \\
\hline $\begin{array}{l}\text { 4141. } 400 \\
4139.718 \\
4139.276 \\
4137.642 \\
4136.200\end{array}$ & $\begin{array}{l}1 n \\
1 \\
1 \\
1 \\
1 n\end{array}$ & $\begin{array}{l}24139.62 \\
24149.42 \\
24152.00 \\
24161.54 \\
24169.96\end{array}$ & $\begin{array}{c}\mathrm{U} \\
\mathrm{U} \\
{ }_{\mathrm{U}}^{\mathrm{W}}\end{array}$ & $\begin{array}{l}4003.287 \\
3999.398 \\
3996.540 \\
3996.139 \\
3995.456\end{array}$ & $\begin{array}{l}1 n \\
1 \\
1 \\
1 \\
0 n\end{array}$ & $\begin{array}{l}24972.41 \\
24996.70 \\
25014.57 \\
25017.08 \\
25021.36\end{array}$ & \\
\hline $\begin{array}{l}\text { 4135. } 887 \\
4135.607 \\
4135.039 \\
4131.146 \\
4127.252\end{array}$ & $\begin{array}{l}1 \\
1 n \\
1 n \\
1 n \\
1\end{array}$ & $\begin{array}{l}24171.79 \\
24173.43 \\
24176.75 \\
24199.53 \\
24222.36\end{array}$ & $\mathrm{U}$ & $\begin{array}{l}\text { 3993. } 642 \\
3991.395 \\
3983.645 \\
3983.518 \\
3978.247\end{array}$ & $\begin{array}{l}1 \\
1 n \\
0 \\
1 n \\
1\end{array}$ & $\begin{array}{l}25032.72 \\
25046.81 \\
25095.54 \\
25096.34 \\
25129.59\end{array}$ & $\mathrm{U}$ \\
\hline $\begin{array}{l}4127.120 \\
4126.965 \\
4124.332 \\
4119.746 \\
4118.065\end{array}$ & $\begin{array}{l}1 \\
1 \\
0 n \\
1 n \\
1 n\end{array}$ & $\begin{array}{l}24223.14 \\
24224.05 \\
24239.51 \\
24266.50 \\
24276.40\end{array}$ & $\mathrm{U}$ & $\begin{array}{l}3977.420 \\
3970.863 \\
3966.973 \\
3962.717 \\
3960.642\end{array}$ & $\begin{array}{l}1 \\
1 \\
1 n \\
1 n \\
1\end{array}$ & $\begin{array}{l}25134.82 \\
25176.32 \\
25201.01 \\
25228.07 \\
25241.29\end{array}$ & $\mathrm{Z}$ \\
\hline $\begin{array}{l}4110.814 \\
4110.310 \\
4107.160 \\
4107.015 \\
4096.695\end{array}$ & $\begin{array}{l}0 \\
1 \\
1 \\
1 n \\
1\end{array}$ & $\begin{array}{l}24319.22 \\
24322.20 \\
24340.86 \\
24341.72 \\
24403.03\end{array}$ & $\mathrm{U}$ & $\begin{array}{l}\text { 3957. } 836 \\
3946.007 \\
3943.166 \\
\text { 3940. } 422 \\
\text { 3939. } 730\end{array}$ & $\begin{array}{l}1 \\
1 \\
1 n \\
1 \\
1\end{array}$ & $\begin{array}{l}25259.18 \\
25334.90 \\
25353.16 \\
25370.81 \\
25375.27\end{array}$ & $\begin{array}{c}\mathrm{BK}, \underset{\mathrm{U}}{\mathrm{U}}, \mathrm{W} \\
\mathrm{U}\end{array}$ \\
\hline $\begin{array}{l}\text { 4095. } 346 \\
4094.422 \\
4086.406 \\
4081.264 \\
4072.332\end{array}$ & $\begin{array}{l}1 \\
1 \\
1 \\
1 \\
0\end{array}$ & $\begin{array}{l}24411.07 \\
24416.58 \\
24464.48 \\
24495.30 \\
24549.02\end{array}$ & $\begin{array}{c}\mathrm{U} \\
\mathrm{U} \\
\mathrm{U} \\
\mathrm{BK}, \mathrm{U}, \mathrm{W}\end{array}$ & $\begin{array}{l}\text { 3936. } 558 \\
\text { 3934. } 976 \\
\text { 3934. } 356 \\
\text { 3932. } 266 \\
\text { 3931. } 883\end{array}$ & $\begin{array}{l}1 \\
1 \\
1 n \\
1 n \\
0\end{array}$ & $\begin{array}{l}25395.71 \\
25405.92 \\
25409.93 \\
25423.43 \\
25425.91\end{array}$ & $\begin{array}{c}\mathrm{U} \\
\mathrm{Z} \\
\mathrm{U}\end{array}$ \\
\hline $\begin{array}{l}4072.183 \\
4071.282 \\
4071.146 \\
4070.954 \\
4070.554\end{array}$ & $\begin{array}{l}0 \\
0 \\
0 \\
1 \\
0\end{array}$ & $\begin{array}{l}24549.92 \\
24555.35 \\
24556.17 \\
24557.33 \\
24559.74\end{array}$ & $\mathrm{U}$ & $\begin{array}{l}3926.422 \\
3924.032 \\
3915.256 \\
3904.564 \\
3843.528\end{array}$ & $\begin{array}{l}1 n \\
1 n \\
1 \\
1 n \\
0 n\end{array}$ & $\begin{array}{l}25461.27 \\
25476.78 \\
25533.88 \\
25603.80 \\
26010.38\end{array}$ & $\begin{array}{c}\mathrm{U} \\
\mathrm{BK}, \mathrm{U} ?, W\end{array}$ \\
\hline $\begin{array}{l}4070.090 \\
4069.786 \\
4069.700 \\
4069.610 \\
4068.898\end{array}$ & $\begin{array}{l}0 \\
0 \\
0 \\
1 \\
0\end{array}$ & $\begin{array}{l}24562.54 \\
24564.38 \\
24564.90 \\
24565.44 \\
24569.74\end{array}$ & & $\begin{array}{l}\text { 3842. } 796 \\
3842.556 \\
3838.567 \\
\text { 3838. } 201 \\
\text { 3838. } 084\end{array}$ & $\begin{array}{l}0 \\
0 \\
1 \\
0 \\
1\end{array}$ & $\begin{array}{l}26015.34 \\
26016.96 \\
26044.00 \\
26046.48 \\
26047.28\end{array}$ & $\mathrm{U}^{\mathrm{c}}$ \\
\hline $\begin{array}{l}4068.800 \\
4068.704 \\
4068.483 \\
4068.254 \\
4066.768\end{array}$ & $\begin{array}{l}0 \\
0 \\
0 \\
0 \\
1\end{array}$ & $\begin{array}{l}24570.33 \\
24570.91 \\
24572.25 \\
24573.63 \\
24582.61\end{array}$ & & $\begin{array}{l}3837.914 \\
3819.679 \\
3819.354 \\
3819.276 \\
3818.950\end{array}$ & $\begin{array}{l}1 \\
1 \\
0 \\
2 \\
3\end{array}$ & $\begin{array}{l}26048.43 \\
26172.78 \\
26175.01 \\
26175.54 \\
26177.78\end{array}$ & $\mathrm{U}^{\mathrm{U}} \mathrm{W}$ \\
\hline $\begin{array}{l}4061.848 \\
4054.454 \\
4049.924 \\
4041.828 \\
4037.136\end{array}$ & $\begin{array}{l}1 n \\
1 \\
1 \\
1 n \\
1 n\end{array}$ & $\begin{array}{l}24612.38 \\
24657.27 \\
24684.85 \\
24734.29 \\
24763.04\end{array}$ & $\begin{array}{c}\mathrm{BK}, \mathrm{U} \\
\mathrm{U}\end{array}$ & $\begin{array}{l}3818.815 \\
3818.593 \\
3818.141 \\
3818.043 \\
3817.793\end{array}$ & $\begin{array}{l}0 n \\
2 n \\
1 \\
0 \\
2\end{array}$ & $\begin{array}{l}26178.70 \\
26180.23 \\
26183.32 \\
26184.00 \\
26185.71\end{array}$ & $\begin{array}{l}\mathrm{Z} \\
\mathrm{U}\end{array}$ \\
\hline $\begin{array}{l}4036.552 \\
4033.648 \\
4026.770 \\
4022.564 \\
4021.374\end{array}$ & $\begin{array}{l}0 n \\
1 \\
1 \\
0 \\
1\end{array}$ & $\begin{array}{ll}24766.62 \\
24784.45 \\
24826.78 \\
24852.74 \\
24860.10\end{array}$ & $\mathrm{U}$ & $\begin{array}{l}3817.424 \\
3817.268 \\
3817.094 \\
3816.702 \\
3816.594\end{array}$ & $\begin{array}{l}1 \\
0 \\
3 n \\
1 \\
1\end{array}$ & $\begin{array}{l}\text { 26188. } 24 \\
26189.31 \\
26190.51 \\
26193.20 \\
26193.94\end{array}$ & \\
\hline $\begin{array}{l}4021.002 \\
4015.023 \\
4014.340 \\
4014.072 \\
4012.628\end{array}$ & $\begin{array}{l}1 \\
1 \\
1 \\
1 \\
1 n\end{array}$ & $\begin{array}{l}24862.40 \\
24899.42 \\
24903.66 \\
24905.32 \\
24914.28\end{array}$ & $\mathrm{U}$ & $\begin{array}{l}3815.188 \\
3815.021 \\
3814.892 \\
3814.668 \\
3814.268\end{array}$ & $\begin{array}{l}1 n \\
1 \\
1 \\
2 \\
1\end{array}$ & $\begin{array}{l}26203.59 \\
26204.74 \\
26205.62 \\
26207.16 \\
26209.91\end{array}$ & $\mathrm{U}$ \\
\hline $\begin{array}{l}4010.618 \\
4010.522 \\
4009.388 \\
4009.240 \\
4008.531\end{array}$ & $\begin{array}{l}1 \\
1 \\
1 \\
1 n \\
1\end{array}$ & $\begin{array}{l}24926.77 \\
24927.36 \\
24934.41 \\
24935.33 \\
24939.74\end{array}$ & BK, U & $\begin{array}{l}3814.044 \\
3812.761 \\
3812.624 \\
3812.518 \\
3812.306\end{array}$ & $\begin{array}{l}2 \\
2 \\
1 \\
2 \\
1 n\end{array}$ & $\begin{array}{l}26211.45 \\
26220.27 \\
26221.21 \\
26221.94 \\
26223.40\end{array}$ & $\mathrm{U}$ \\
\hline
\end{tabular}


TABLE 4.-New Fe I lines, unclassified-Continued

\begin{tabular}{|c|c|c|c|c|c|c|c|}
\hline $\begin{array}{l}\text { Wavelength } \\
\text { A }\end{array}$ & Intensity & $\begin{array}{l}\text { Wave Number } \\
\mathrm{cm}^{-1}\end{array}$ & $\begin{array}{l}\text { Notes and } \\
\text { References }\end{array}$ & $\begin{array}{c}\text { Wavelength } \\
\text { A }\end{array}$ & Intensity & $\begin{array}{l}\text { Wave Number } \\
\mathrm{cm}^{-1}\end{array}$ & $\begin{array}{l}\text { Notes and } \\
\text { References }\end{array}$ \\
\hline $\begin{array}{l}3812.026 \\
3811.484 \\
3811.101 \\
3810.526 \\
3810.332\end{array}$ & $\begin{array}{l}2 \\
3 \\
1 \\
2 \\
0 n\end{array}$ & $\begin{array}{l}26225.33 \\
26229.06 \\
26231.69 \\
26235.65 \\
26236.98\end{array}$ & $\begin{array}{l}\mathrm{U} \\
\mathrm{W} \\
\mathrm{U} \\
\mathrm{U}\end{array}$ & $\begin{array}{l}3791.930 \\
3790.917 \\
3790.254 \\
3788.944 \\
3788.593\end{array}$ & $\begin{array}{l}0 \\
0 n \\
3 \\
1 n \\
0\end{array}$ & $\begin{array}{l}26364.31 \\
26371.35 \\
26375.97 \\
26385.08 \\
26387.53\end{array}$ & $\begin{array}{l}\mathrm{U} \\
\mathrm{U}\end{array}$ \\
\hline $\begin{array}{l}\text { 3809. } 882 \\
3809.334 \\
3808.871 \\
\text { 3807. } 986 \\
\text { 3807. } 791\end{array}$ & $\begin{array}{l}0 \\
0 \\
0 \\
1 \\
0 n\end{array}$ & $\begin{array}{l}26240.08 \\
26243.86 \\
26247.05 \\
26253.15 \\
26254.49\end{array}$ & $\mathrm{U}$ & $\begin{array}{l}3788.472 \\
3788.281 \\
3787.661 \\
3787.379 \\
3787.059\end{array}$ & $\begin{array}{l}0 \\
1 \\
1 \\
0 \\
0\end{array}$ & $\begin{array}{l}\text { 26388. } 37 \\
26389.70 \\
26394.02 \\
26395.99 \\
26398.22\end{array}$ & $\mathrm{U}$ \\
\hline $\begin{array}{l}3807.313 \\
3807.088 \\
3806.476 \\
3806.052 \\
\text { 3805. } 771\end{array}$ & $\begin{array}{l}0 \\
0 \\
0 \\
0 \\
3\end{array}$ & $\begin{array}{l}\text { 26257. } 79 \\
26259.34 \\
26263.56 \\
26266.49 \\
26268.43\end{array}$ & $\begin{array}{l}\mathrm{U} \\
\mathrm{W} \\
\mathrm{U}\end{array}$ & $\begin{array}{l}3786.907 \\
3786.811 \\
3786.443 \\
3785.486 \\
3785.262\end{array}$ & $\begin{array}{l}0 \\
2 \\
3 \\
3 \\
0\end{array}$ & $\begin{array}{l}26399.28 \\
26399.95 \\
26402.51 \\
26409.19 \\
26410.75\end{array}$ & $\mathrm{U}$ \\
\hline $\begin{array}{l}3805.568 \\
3805.478 \\
3805.140 \\
3805.024 \\
3804.842\end{array}$ & $\begin{array}{l}0 \\
1 \\
1 \\
1 \\
0 n\end{array}$ & $\begin{array}{l}26269.83 \\
26270.45 \\
26272.78 \\
26273.58 \\
26274.84\end{array}$ & $\mathrm{U}$ & $\begin{array}{l}3785.127 \\
3784.934 \\
3784.812 \\
3784.698 \\
3784.386\end{array}$ & $\begin{array}{l}0 \\
0 \\
1 \\
1 \\
3 n\end{array}$ & $\begin{array}{l}26411.69 \\
26413.04 \\
26413.89 \\
26414.68 \\
26416.86\end{array}$ & $\mathrm{U}$ \\
\hline $\begin{array}{ll}3804 . & 501 \\
3804 . & 228 \\
3803 . & 704 \\
\text { 3803. } 408 \\
\text { 3803. } 106\end{array}$ & $\begin{array}{l}1 \\
0 \\
0 \\
3 \\
1 n\end{array}$ & $\begin{array}{l}26277.20 \\
26279.08 \\
26282.70 \\
26284.75 \\
26286.84\end{array}$ & $\mathrm{U}$ & $\begin{array}{l}3783.721 \\
3783.470 \\
3783.275 \\
3782.862 \\
3781.749\end{array}$ & $\begin{array}{l}2 \\
0 \\
1 \\
3 \\
0\end{array}$ & $\begin{array}{l}26421.51 \\
26423.26 \\
26424.62 \\
26427.50 \\
26435.28\end{array}$ & $\mathrm{U}$ \\
\hline $\begin{array}{l}\text { 3802. } 936 \\
3802.682 \\
3802.536 \\
3801.468 \\
3801.066\end{array}$ & $\begin{array}{l}0 n \\
1 \\
0 \\
1 \\
0\end{array}$ & $\begin{array}{l}26288.01 \\
26289.76 \\
26290.78 \\
26298.16 \\
26300.94\end{array}$ & $\mathrm{U}$ & $\begin{array}{l}3781.300 \\
3780.966 \\
3780.828 \\
3780.678 \\
3780.562\end{array}$ & $\begin{array}{l}0 \\
0 \\
0 \\
0 \\
0\end{array}$ & $\begin{array}{l}26438.42 \\
26440.76 \\
26441.72 \\
26442.77 \\
26443.58\end{array}$ & \\
\hline $\begin{array}{l}3800.955 \\
3800.813 \\
3800.699 \\
3800.407 \\
3800.269\end{array}$ & $\begin{array}{l}0 \\
0 n \\
2 \\
1 \\
1\end{array}$ & $\begin{array}{l}\text { 26301. } 71 \\
26302.69 \\
26303.48 \\
26305.50 \\
26306.46\end{array}$ & $\mathrm{U}$ & $\begin{array}{l}3780.256 \\
3780.004 \\
3779.810 \\
3778.928 \\
3778.809\end{array}$ & $\begin{array}{l}0 \\
0 \\
0 \\
0 \\
1\end{array}$ & $\begin{array}{l}\text { 26445. } 72 \\
26447.49 \\
26448.84 \\
26455.02 \\
26455.85\end{array}$ & $\begin{array}{l}\mathrm{U} \\
\mathrm{U} \\
\mathrm{U}\end{array}$ \\
\hline $\begin{array}{l}3800.173 \\
3800.026 \\
3799.208 \\
3799.109 \\
3799.024\end{array}$ & $\begin{array}{l}0 \\
0 \\
1 \\
1 \\
0\end{array}$ & $\begin{array}{l}\text { 26307. } 12 \\
\text { 26308. } 14 \\
\text { 26313. } 80 \\
\text { 26314. } 49 \\
26315.08\end{array}$ & & $\begin{array}{l}3777.848 \\
3777.588 \\
3777.186 \\
3776.838 \\
3776.226\end{array}$ & $\begin{array}{l}1 \\
0 n \\
3 \\
0 \\
0\end{array}$ & $\begin{array}{l}26462.58 \\
26464.40 \\
26467.22 \\
26469.66 \\
26473.94\end{array}$ & $\mathrm{U}$ \\
\hline $\begin{array}{l}3798.910 \\
3798.196 \\
3798.072 \\
3797.666 \\
3797.191\end{array}$ & $\begin{array}{l}1 \\
0 \\
1 \\
3 \\
0\end{array}$ & $\begin{array}{l}26315.87 \\
26320.82 \\
26321.68 \\
26324.49 \\
26327.78\end{array}$ & $\mathrm{U}$ & $\begin{array}{l}3776.110 \\
3775.972 \\
3775.656 \\
3775.503 \\
3775.364\end{array}$ & $\begin{array}{l}0 \\
0 \\
2 \\
0 \\
0\end{array}$ & $\begin{array}{l}\text { 26474. } 76 \\
26475.73 \\
26477.94 \\
26479.02 \\
26479.99\end{array}$ & \\
\hline $\begin{array}{l}3797.054 \\
3796.730 \\
3796.608 \\
3796.259 \\
3795.824\end{array}$ & $\begin{array}{l}0 \\
0 \\
1 n \\
0 \\
0\end{array}$ & $\begin{array}{l}\text { 26328. } 73 \\
26330.98 \\
26331.82 \\
26334.24 \\
26337.26\end{array}$ & $\begin{array}{c}\mathrm{U}, \mathrm{W} \\
\mathrm{U}\end{array}$ & $\begin{array}{l}3775.080 \\
3774.952 \\
3774.180 \\
3773.999 \\
3773.470\end{array}$ & $\begin{array}{l}0 \\
1 \\
0 \\
0 \\
0\end{array}$ & $\begin{array}{l}\text { 26481. } 98 \\
26482.88 \\
26489.57 \\
26493.28 \\
26494.16\end{array}$ & $\mathrm{U}$ \\
\hline $\begin{array}{l}3795.741 \\
3795.608 \\
3795.386 \\
3794.562 \\
3794.486\end{array}$ & $\begin{array}{l}1 \\
0 \\
1 \\
1 \\
1\end{array}$ & $\begin{array}{l}26337.84 \\
26338.76 \\
26340.30 \\
26346.02 \\
26346.55\end{array}$ & $\mathrm{U}$ & $\begin{array}{l}3773.166 \\
3772.841 \\
3772.702 \\
3772.287 \\
3771.816\end{array}$ & $\begin{array}{l}1 \\
0 \\
0 \\
0 \\
1 n\end{array}$ & $\begin{array}{l}26496.75 \\
26497.70 \\
26498.67 \\
26501.59 \\
26504.90\end{array}$ & $\mathrm{U}$ \\
\hline $\begin{array}{l}3794.174 \\
3793.726 \\
3793.136 \\
3792.454 \\
3792.292\end{array}$ & $\begin{array}{l}0 \\
3 \\
1 \\
0 \\
0\end{array}$ & $\begin{array}{l}26348.72 \\
26351.83 \\
26355.93 \\
26360.67 \\
26361.79\end{array}$ & ${ }_{U}^{U}, W$ & $\begin{array}{l}3771.249 \\
3770.786 \\
3770.548 \\
3769.310 \\
3769.071\end{array}$ & $\begin{array}{l}0 n \\
0 \\
0 \\
1 n \\
1 n\end{array}$ & $\begin{array}{l}26508.88 \\
26512.14 \\
26513.81 \\
26522.52 \\
26524.20\end{array}$ & \\
\hline
\end{tabular}


TABLE 4.-New Fe I lines, unclassified-Continued

\begin{tabular}{|c|c|c|c|c|c|c|c|}
\hline $\begin{array}{c}\text { Wavelength } \\
\text { A }\end{array}$ & Intensity & $\begin{array}{l}\text { Wave Number } \\
\mathrm{cm}^{-1}\end{array}$ & $\begin{array}{l}\text { Notes and } \\
\text { References }\end{array}$ & $\begin{array}{c}\text { Wavelength } \\
\mathrm{A}\end{array}$ & Intensity & $\begin{array}{l}\text { Wave Number } \\
\mathrm{cm}^{-1}\end{array}$ & $\begin{array}{l}\text { Notes and } \\
\text { References }\end{array}$ \\
\hline $\begin{array}{l}3768.697 \\
3768.561 \\
3767.788 \\
3767.610 \\
3764.718\end{array}$ & $\begin{array}{l}0 \\
3 \\
1 \\
3 \\
0\end{array}$ & $\begin{array}{l}26526.83 \\
26527.79 \\
26533.23 \\
26534.49 \\
26554.87\end{array}$ & $\mathrm{U}$ & $\begin{array}{l}\text { 3614. } 272 \\
3613.296 \\
3612.783 \\
3612.671 \\
3612.418\end{array}$ & $\begin{array}{l}1 \\
0 \\
1 \\
0 \\
0\end{array}$ & $\begin{array}{l}27660.20 \\
27667.67 \\
27671.60 \\
27672.46 \\
27674.40\end{array}$ & BK \\
\hline $\begin{array}{l}3759.732 \\
3754.068 \\
3741.903 \\
3741.743 \\
3728.972\end{array}$ & $\begin{array}{l}1 \\
0 \\
0 n \\
0 n \\
2\end{array}$ & $\begin{array}{l}26590.08 \\
26630.20 \\
26716.77 \\
26717.92 \\
26809.42\end{array}$ & $\begin{array}{l}\mathrm{Z} ? \\
\mathrm{U}\end{array}$ & $\begin{array}{l}3612.233 \\
3611.935 \\
3611.768 \\
3611.651 \\
3611.519\end{array}$ & $\begin{array}{l}2 \\
1 n \\
1 \\
1 n \\
1\end{array}$ & $\begin{array}{l}27675.81 \\
27678.10 \\
27679.38 \\
27680.27 \\
27681.28\end{array}$ & \\
\hline $\begin{array}{l}3723.946 \\
3723.570 \\
3706.070 \\
3699.810 \\
3695.632\end{array}$ & $\begin{array}{l}1 \\
0 n \\
1 \\
1 \\
2 n\end{array}$ & $\begin{array}{l}26845.60 \\
26848.31 \\
26975.08 \\
27020.72 \\
27051.27\end{array}$ & $\begin{array}{l}\mathrm{U} \\
\mathrm{U} \\
\mathrm{Z}\end{array}$ & $\begin{array}{l}3611.392 \\
3611.188 \\
3610.972 \\
3610.867 \\
3609.996\end{array}$ & $\begin{array}{l}0 n \\
1 n \\
0 \\
0 \\
1\end{array}$ & $\begin{array}{l}27682.26 \\
27683.82 \\
27685.48 \\
27686.28 \\
27692.96\end{array}$ & $\mathrm{U}$ \\
\hline $\begin{array}{l}3694.752 \\
3680.962 \\
3680.396 \\
3676.008 \\
3672.114\end{array}$ & $\begin{array}{l}1 \\
2 n \\
2 n \\
0 \\
0\end{array}$ & $\begin{array}{l}27057.72 \\
27159.08 \\
27163.26 \\
27195.68 \\
27224.52\end{array}$ & $\begin{array}{l}\mathrm{U} \\
\mathrm{Z} \\
\mathrm{U}\end{array}$ & $\begin{array}{l}3609.884 \\
3609.426 \\
3608.365 \\
\text { 3607. } 780 \\
3607.634\end{array}$ & $\begin{array}{l}1 n \\
1 \\
1 \\
2 n \\
1 n\end{array}$ & $\begin{array}{l}27693.82 \\
27697.24 \\
27705.48 \\
27709.97 \\
27711.09\end{array}$ & $\mathrm{U}$ \\
\hline $\begin{array}{l}\text { 3668. } 730 \\
3665.845 \\
3665.762 \\
3659.214 \\
3659.094\end{array}$ & $\begin{array}{l}1 n \\
1 \\
1 \\
1 \\
1 n\end{array}$ & $\begin{array}{l}27249.63 \\
27271.07 \\
27271.69 \\
27320.49 \\
27321.39\end{array}$ & $\begin{array}{l}\mathrm{U}, \mathrm{W} \\
\mathrm{U}, \mathrm{W}\end{array}$ & $\begin{array}{l}\text { 3607. } 333 \\
3607.256 \\
3607.102 \\
\text { 3606. } 932 \\
\text { 3606. } 253\end{array}$ & $\begin{array}{l}1 \\
1 \\
2 n \\
1 \\
2\end{array}$ & $\begin{array}{l}27713.41 \\
27714.00 \\
27715.18 \\
27716.49 \\
27721.70\end{array}$ & $\mathrm{U}$ \\
\hline $\begin{array}{l}3658.742 \\
3653.157 \\
3652.969 \\
3651.918 \\
3651.182\end{array}$ & $\begin{array}{l}1 \\
0 n \\
0 \\
1 n \\
1 n\end{array}$ & $\begin{array}{l}27324.02 \\
27365.79 \\
27367.20 \\
27375.07 \\
27380.59\end{array}$ & $\begin{array}{l}\mathrm{U} \\
\mathrm{U} \\
\mathrm{U}\end{array}$ & $\begin{array}{l}3606.165 \\
3606.016 \\
3604.874 \\
3604.090 \\
3603.956\end{array}$ & $\begin{array}{l}1 \\
0 \\
0 \\
0 n \\
2\end{array}$ & $\begin{array}{l}27722.38 \\
27723.53 \\
27732.31 \\
27738.34 \\
27739.37\end{array}$ & $\mathrm{Z}$ \\
\hline $\begin{array}{l}3646.580 \\
3642.198 \\
3640.834 \\
3640.096 \\
3639.964\end{array}$ & $\begin{array}{l}1 n \\
0 n \\
1 \\
1 \\
1 n\end{array}$ & $\begin{array}{l}27415.14 \\
27448.13 \\
27458.41 \\
27463.98 \\
27464.97\end{array}$ & $\begin{array}{l}\mathrm{U} \\
\mathrm{U}\end{array}$ & $\begin{array}{l}3603.449 \\
3603.350 \\
3602.898 \\
3602.323 \\
3602.223\end{array}$ & $\begin{array}{l}1 \\
2 \\
0 \\
0 \\
0\end{array}$ & $\begin{array}{l}27743.28 \\
27744.04 \\
27747.52 \\
27751.95 \\
27752.72\end{array}$ & \\
\hline $\begin{array}{l}3639.605 \\
3639.502 \\
3639.308 \\
3628.868 \\
3628.620\end{array}$ & $\begin{array}{l}1 n \\
1 n \\
1 n \\
1 \\
1\end{array}$ & $\begin{array}{l}27467.68 \\
27468.46 \\
27469.92 \\
27548.95 \\
27550.83\end{array}$ & $\begin{array}{l}\mathrm{U} \\
\mathrm{U} \\
\mathrm{W}\end{array}$ & $\begin{array}{l}3601.858 \\
3601.273 \\
3601.116 \\
3600.870 \\
3600.675\end{array}$ & $\begin{array}{l}1 \\
1 \\
1 \\
1 \\
1\end{array}$ & $\begin{array}{ll}27755 . & 53 \\
27760 . & 04 \\
27761 . & 25 \\
27763 . & 15 \\
27764 . & 65\end{array}$ & $\mathrm{U}$ \\
\hline $\begin{array}{l}3628.414 \\
3628.210 \\
3627.419 \\
3625.498 \\
3622.981\end{array}$ & $\begin{array}{l}0 \\
0 \\
1 n \\
1 \\
1\end{array}$ & $\begin{array}{l}27552.40 \\
27553.94 \\
27559.95 \\
27574.56 \\
27593.71\end{array}$ & $\mathrm{~S}, \mathrm{U}$ & $\begin{array}{l}3600.533 \\
3600.418 \\
3600.036 \\
3599.972 \\
3599.842\end{array}$ & $\begin{array}{l}1 \\
1 \\
1 n \\
2 n \\
1\end{array}$ & $\begin{array}{l}27765.74 \\
27766.63 \\
27769.58 \\
27770.07 \\
27771.07\end{array}$ & $\begin{array}{l}\mathrm{U} \\
\mathrm{U} \\
\mathrm{U} \\
\mathrm{Z}\end{array}$ \\
\hline $\begin{array}{l}3622.751 \\
3622.431 \\
3620.679 \\
3619.942 \\
3617.629\end{array}$ & $\begin{array}{l}1 n \\
3 \\
1 \\
2 n \\
1 n\end{array}$ & $\begin{array}{l}27595.46 \\
27597.90 \\
27611.26 \\
27616.88 \\
27634.53\end{array}$ & $\mathrm{U}$ & $\begin{array}{l}\text { 3599. } 760 \\
\text { 3599. } 468 \\
3599.293 \\
\text { 3598. } 554 \\
\text { 3598. } 233\end{array}$ & $\begin{array}{l}1 \\
0 \\
0 \\
0 \\
1 n\end{array}$ & $\begin{array}{l}27771.71 \\
27773.96 \\
27775.31 \\
27781.01 \\
27783.49\end{array}$ & $\mathrm{U}$ \\
\hline $\begin{array}{l}3617.472 \\
3617.007 \\
3616.857 \\
3616.722 \\
3616.642\end{array}$ & $\begin{array}{l}1 \\
2 \\
0 \\
1 \\
2\end{array}$ & $\begin{array}{l}27635.73 \\
27639.29 \\
27640.43 \\
27641.46 \\
27642.08\end{array}$ & $\mathrm{BK}, \mathrm{U}$ & $\begin{array}{l}3597.804 \\
3597.478 \\
3597.314 \\
3597.158 \\
3596.853\end{array}$ & $\begin{array}{l}1 \\
0 \\
0 \\
0 \\
1 n\end{array}$ & $\begin{array}{l}27786.80 \\
27789.32 \\
27790.59 \\
27791.79 \\
27794.15\end{array}$ & $\mathrm{BK}, \mathrm{W}$ \\
\hline $\begin{array}{l}\text { 3616. } 036 \\
3615.814 \\
3615.518 \\
3615.328 \\
3614.404\end{array}$ & $\begin{array}{l}0 \\
2 \\
0 \\
0 \\
0 n\end{array}$ & $\begin{array}{l}27646.71 \\
27648.40 \\
27650.67 \\
27652.12 \\
27659.91\end{array}$ & $\begin{array}{c}\mathrm{U}, \underset{\mathrm{U}}{\mathrm{W}} \\
\mathrm{Z}\end{array}$ & $\begin{array}{l}3596.727 \\
3596.612 \\
3596.438 \\
3596.334 \\
\text { 3595. } 526\end{array}$ & $\begin{array}{l}0 \\
0 \\
0 \\
0 \\
1\end{array}$ & $\begin{array}{l}27795.12 \\
27796.01 \\
27797.36 \\
27798.16 \\
27804.41\end{array}$ & $\mathrm{U}$ \\
\hline
\end{tabular}


TABLE 4.-New Fe I lines, unclassified-Continued

\begin{tabular}{|c|c|c|c|c|c|c|c|}
\hline $\begin{array}{c}\text { Wavelength } \\
\mathrm{A}\end{array}$ & Intensity & $\begin{array}{l}\text { Wave Number } \\
\mathrm{cm}^{-1}\end{array}$ & $\begin{array}{l}\text { Notes and } \\
\text { References }\end{array}$ & $\begin{array}{c}\text { Wavelength } \\
\text { A }\end{array}$ & Intensity & $\begin{array}{l}\text { Wave Number } \\
\mathrm{cm}^{-1}\end{array}$ & $\begin{array}{l}\text { Notes and } \\
\text { References }\end{array}$ \\
\hline $\begin{array}{l}3595.438 \\
3594.427 \\
3594.312 \\
3594.176 \\
\text { 3593. } 860\end{array}$ & $\begin{array}{l}0 \\
1 \\
0 \\
1 \\
0\end{array}$ & $\begin{array}{l}27805.09 \\
27812.91 \\
27813.80 \\
27814.85 \\
27817.30\end{array}$ & $\mathrm{U}$ & $\begin{array}{l}3486.142 \\
3485.766 \\
3484.586 \\
3483.890 \\
3482.446\end{array}$ & $\begin{array}{l}1 \\
1 \\
2 \\
2 \\
1\end{array}$ & $\begin{array}{l}28676.80 \\
28679.89 \\
28689.60 \\
28695.33 \\
28707.23\end{array}$ & $\begin{array}{l}\left({ }^{c}\right) \\
\mathrm{U} \\
(\mathrm{c})\end{array}$ \\
\hline $\begin{array}{l}3593.119 \\
3592.354 \\
3591.940 \\
3591.174 \\
3590.500\end{array}$ & $\begin{array}{l}2 \\
1 \\
0 \\
0 \\
1\end{array}$ & $\begin{array}{l}27823.03 \\
27828.96 \\
27832.17 \\
27838.10 \\
27843.33\end{array}$ & & $\begin{array}{l}3472.318 \\
3457.894 \\
3455.726 \\
3455.393 \\
3450.743\end{array}$ & $\begin{array}{l}0 n \\
1 \\
0 \\
1 n \\
1\end{array}$ & $\begin{array}{l}28790.96 \\
28911.05 \\
28929.19 \\
28931.98 \\
28970.96\end{array}$ & $\mathrm{U}, \mathrm{W}$ \\
\hline $\begin{array}{ll}3590 . & 422 \\
3590 . & 204 \\
3589.876 \\
\text { 3589. } 224 \\
\text { 3588. } 724\end{array}$ & $\begin{array}{l}0 \\
0 \\
0 n \\
2 \\
0\end{array}$ & $\begin{array}{l}27843.93 \\
27845.62 \\
27848.17 \\
27853.23 \\
27857.11\end{array}$ & & $\begin{array}{l}3448.606 \\
3447.700 \\
3444.532 \\
3435.219 \\
3434.182\end{array}$ & $\begin{array}{l}1 n \\
1 \\
2 \\
0 n \\
0\end{array}$ & $\begin{array}{l}28988.92 \\
28996.54 \\
29023.20 \\
29101.88 \\
29110.67\end{array}$ & \\
\hline $\begin{array}{l}\text { 3588. } 284 \\
\text { 3587. } 844 \\
\text { 3587. } 604 \\
\text { 3587. } 527 \\
\text { 3587. } 328\end{array}$ & $\begin{array}{l}2 \\
0 \\
0 \\
1 \\
3\end{array}$ & $\begin{array}{l}27860.52 \\
27863.94 \\
27865.80 \\
27866.40 \\
27867.95\end{array}$ & & $\begin{array}{l}3430.554 \\
3430.066 \\
3429.179 \\
3425.441 \\
3423.558\end{array}$ & $\begin{array}{l}1 n \\
0 n \\
1 \\
1 \\
1\end{array}$ & $\begin{array}{l}29141.46 \\
29145.60 \\
29153.14 \\
29184.95 \\
29201.00\end{array}$ & \\
\hline $\begin{array}{l}\text { 3586. } 390 \\
3586.332 \\
3584.468 \\
3584.354 \\
3584.264\end{array}$ & $\begin{array}{l}0 n \\
0 \\
0 \\
1 \\
1 n\end{array}$ & $\begin{array}{l}27875.24 \\
27875.69 \\
27890.18 \\
27891.07 \\
27891.77\end{array}$ & $\mathrm{U}$ & $\begin{array}{l}3422.120 \\
3421.930 \\
3420.864 \\
3420.250 \\
3419.258\end{array}$ & $\begin{array}{l}2 \\
1 \\
1 \\
0 n \\
1 n\end{array}$ & $\begin{array}{l}\text { 29213. } 27 \\
29214.90 \\
29224.00 \\
29229.25 \\
29237.73\end{array}$ & $\mathrm{Z}$ \\
\hline $\begin{array}{l}\text { 3584. } 110 \\
3583.921 \\
\text { 3583. } 687 \\
\text { 3583. } 577 \\
\text { 3583. } 036\end{array}$ & $\begin{array}{l}1 \\
1 n \\
2 \\
1 \\
0\end{array}$ & $\begin{array}{l}27892.97 \\
27894.44 \\
27896.26 \\
27897.12 \\
27901.33\end{array}$ & $\mathrm{Z}$ & $\begin{array}{l}3416.840 \\
3416.562 \\
3414.432 \\
3412.418 \\
3412.134\end{array}$ & $\begin{array}{l}1 \\
1 \\
0 \\
1 \\
1\end{array}$ & $\begin{array}{l}29258.42 \\
29260.80 \\
29279.05 \\
29296.33 \\
29298.77\end{array}$ & $\mathrm{U}$ \\
\hline $\begin{array}{l}\text { 3582. } 970 \\
3582.908 \\
3582.460 \\
3582.032 \\
3581.951\end{array}$ & $\begin{array}{l}3 n \\
1 \\
2 \\
1 n \\
1\end{array}$ & $\begin{array}{l}27901.84 \\
27902.32 \\
27905.81 \\
27909.15 \\
27909.78\end{array}$ & & $\begin{array}{l}3409.742 \\
3409.461 \\
3408.474 \\
3402.743 \\
3398.945\end{array}$ & $\begin{array}{l}1 \\
0 \\
1 \\
1 n \\
1 n\end{array}$ & $\begin{array}{l}29319.32 \\
29321.74 \\
29330.23 \\
29379.62 \\
29412.45\end{array}$ & $\begin{array}{c}\mathrm{U} \\
\mathrm{U}, \mathrm{W} \\
\mathrm{U}\end{array}$ \\
\hline $\begin{array}{l}3580.402 \\
3579.562 \\
3577.490 \\
3574.609 \\
3555.736\end{array}$ & $\begin{array}{l}1 \\
1 \\
1 n \\
2 \\
1\end{array}$ & $\begin{array}{l}27921.85 \\
27928.41 \\
27944.58 \\
27967.10 \\
28115.54\end{array}$ & $\begin{array}{l}\mathrm{U} \\
\mathrm{Z}\end{array}$ & $\begin{array}{l}3398.620 \\
3398.374 \\
3395.692 \\
3395.436 \\
3393.053\end{array}$ & $\begin{array}{l}1 \\
1 \\
0 \\
0 \\
1 n\end{array}$ & $\begin{array}{l}29415.26 \\
29417.40 \\
29440.63 \\
29442.85 \\
29463.53\end{array}$ & $\stackrel{(\mathrm{c})}{\mathrm{U}}$ \\
\hline $\begin{array}{l}3550.309 \\
3550.189 \\
3539.376 \\
3538.688 \\
3525.622\end{array}$ & $\begin{array}{l}0 \\
0 \\
1 \\
1 \\
1\end{array}$ & $\begin{array}{l}28158.52 \\
28159.47 \\
28245.50 \\
28250.99 \\
28355.68\end{array}$ & $\underset{\mathrm{U}}{\mathrm{BK}}$ & $\begin{array}{l}3390.218 \\
3387.558 \\
3384.946 \\
3384.392 \\
3381.132\end{array}$ & $\begin{array}{l}0 \\
1 \\
0 n \\
0 \\
1\end{array}$ & $\begin{array}{l}29488.16 \\
29511.32 \\
29534.09 \\
29538.92 \\
29567.40\end{array}$ & \\
\hline $\begin{array}{l}3520.668 \\
3520.214 \\
3519.500 \\
3519.350 \\
3515.534\end{array}$ & $\begin{array}{l}0 \\
0 \\
1 \\
0 \\
1\end{array}$ & $\begin{array}{l}28395.58 \\
28399.24 \\
28405.00 \\
28406.22 \\
28437.05\end{array}$ & $\mathrm{U}$ & $\begin{array}{l}3380.756 \\
3379.688 \\
3379.409 \\
3377.971 \\
3377.833\end{array}$ & $\begin{array}{l}1 \\
1 \\
1 n \\
3 \\
0\end{array}$ & $\begin{array}{l}29570.69 \\
29580.04 \\
29582.48 \\
29595.07 \\
29596.28\end{array}$ & $\begin{array}{l}\mathrm{U} \\
\mathrm{U} \\
\mathrm{V}\end{array}$ \\
\hline $\begin{array}{l}3515.286 \\
3510.682 \\
3506.946 \\
3506.092 \\
3501.375\end{array}$ & $\begin{array}{l}1 \\
2 \\
1 \\
1 \\
1\end{array}$ & $\begin{array}{l}28439.06 \\
28476.35 \\
28506.68 \\
28513.63 \\
28552.04\end{array}$ & $\begin{array}{c}\text { BK, } \underset{U}{\mathrm{U}}, \mathrm{W} \\
\mathrm{U}\end{array}$ & $\begin{array}{l}3377.345 \\
3377.006 \\
3375.372 \\
3375.046 \\
3373.750\end{array}$ & $\begin{array}{l}0 n \\
0 n \\
2 \\
1 \\
0\end{array}$ & $\begin{array}{l}29600.56 \\
29603.53 \\
29617.86 \\
29620.72 \\
29632.10\end{array}$ & $\begin{array}{l}\mathrm{V} \\
\mathrm{U}\end{array}$ \\
\hline $\begin{array}{l}3499.271 \\
3499.154 \\
3492.310 \\
3491.780 \\
3488.827\end{array}$ & $\begin{array}{l}1 \\
1 \\
1 n \\
0 \\
2\end{array}$ & $\begin{array}{l}28569.21 \\
28570.16 \\
28626.15 \\
28630.50 \\
28654.73\end{array}$ & $\mathrm{U}$ & $\begin{array}{l}3373.300 \\
3371.928 \\
3371.526 \\
3371.304 \\
3371.200\end{array}$ & $\begin{array}{l}1 \\
1 \\
2 \\
1 \\
1\end{array}$ & $\begin{array}{l}29636.05 \\
29648.11 \\
29651.64 \\
29653.60 \\
29654.51\end{array}$ & $\mathrm{U}$ \\
\hline
\end{tabular}


TaвLE 4.-New Fe I lines, unclassified-Continued

\begin{tabular}{|c|c|c|c|c|c|c|c|}
\hline $\begin{array}{c}\text { Wavelength } \\
\text { A }\end{array}$ & Intensity & $\begin{array}{c}\text { Wave Number } \\
\mathrm{cm}^{-1}\end{array}$ & $\begin{array}{l}\text { Notes and } \\
\text { References }\end{array}$ & $\begin{array}{c}\text { Wavelength } \\
\mathrm{A}\end{array}$ & Intensity & $\begin{array}{l}\text { Wave Number } \\
\mathrm{cm}^{-1}\end{array}$ & $\begin{array}{l}\text { Notes and } \\
\text { References }\end{array}$ \\
\hline $\begin{array}{l}3369.958 \\
3368.800 \\
3368.556 \\
3368.435 \\
3367.660\end{array}$ & $\begin{array}{l}0 \\
1 n \\
0 \\
0 \\
1\end{array}$ & $\begin{array}{l}29665.44 \\
29675.64 \\
29677.78 \\
29678.85 \\
29685.68\end{array}$ & $\begin{array}{l}\text { (c) } \\
\mathrm{U}\end{array}$ & $\begin{array}{l}3262.878 \\
3262.132 \\
3261.636 \\
3261.425 \\
3261.146\end{array}$ & $\begin{array}{l}1 \\
0 \\
0 \\
0 \\
0\end{array}$ & $\begin{array}{l}30638.96 \\
30645.96 \\
30650.62 \\
20652.60 \\
30655.23\end{array}$ & $\mathrm{U}$ \\
\hline $\begin{array}{l}3367.292 \\
3366.494 \\
3364.492 \\
3359.608 \\
3359.390\end{array}$ & $\begin{array}{l}1 \\
0 \\
0 \\
1 \\
1\end{array}$ & $\begin{array}{l}\text { 29688. } 92 \\
29695.96 \\
29713.63 \\
29756.83 \\
29758.76\end{array}$ & $\underset{\mathrm{U}}{\mathrm{U}}$ & $\begin{array}{l}3260.880 \\
3260.723 \\
3260.624 \\
3260.549 \\
3260.460\end{array}$ & $\begin{array}{l}0 \\
0 \\
0 \\
0 \\
0\end{array}$ & $\begin{array}{l}30657.73 \\
30659.20 \\
30660.14 \\
30660.84 \\
30661.68\end{array}$ & $\mathrm{U}$ \\
\hline $\begin{array}{l}3358.573 \\
3358.386 \\
3358.320 \\
3358.044 \\
3357.558\end{array}$ & $\begin{array}{l}1 \\
2 n \\
1 \\
0 \\
1\end{array}$ & $\begin{array}{l}29766.00 \\
29767.65 \\
29768.24 \\
29770.68 \\
29774.99\end{array}$ & $Z$ & $\begin{array}{l}3259.708 \\
3258.092 \\
3255.091 \\
3250.212 \\
3249.844\end{array}$ & $\begin{array}{l}1 \\
1 \\
0 \\
0 n \\
1\end{array}$ & $\begin{array}{l}30668.75 \\
30683.96 \\
30712.25 \\
30758.35 \\
30761.83\end{array}$ & $\begin{array}{c}\mathrm{U} \\
\mathrm{U} \\
(\mathrm{c}) \\
\mathrm{U} \\
\mathrm{W}, \mathrm{Z}\end{array}$ \\
\hline $\begin{array}{l}3354.854 \\
3354.512 \\
3354.176 \\
3352.004 \\
3351.854\end{array}$ & $\begin{array}{l}1 n \\
1 \\
1 \\
1 \\
1\end{array}$ & $\begin{array}{l}\text { 29798. } 99 \\
\text { 29802. } 03 \\
\text { 29805. } 02 \\
29824.33 \\
\text { 29825. } 66\end{array}$ & $\mathrm{U}$ & $\begin{array}{l}3241.378 \\
3232.656 \\
3232.347 \\
3222.808 \\
3220.486\end{array}$ & $\begin{array}{l}1 n \\
1 \\
0 \\
0 n \\
1 n\end{array}$ & $\begin{array}{l}30842.18 \\
30925.39 \\
30928.34 \\
31019.88 \\
31042.25\end{array}$ & $\begin{array}{l}\mathrm{U} \\
\mathrm{U} \\
\mathrm{U} \\
\mathrm{U}\end{array}$ \\
\hline $\begin{array}{l}3345.234 \\
3341.626 \\
3341.507 \\
3340.292 \\
3340.184\end{array}$ & $\begin{array}{l}0 \\
0 \\
0 \\
0 \\
0\end{array}$ & $\begin{array}{l}\text { 29884. } 68 \\
29916.95 \\
\text { 29918. } 01 \\
29928.90 \\
\text { 29929. } 86\end{array}$ & $\mathrm{U}$ & $\begin{array}{l}3216.581 \\
3216.343 \\
3204.454 \\
3203.677 \\
3202.958\end{array}$ & $\begin{array}{l}0 n \\
0 \\
0 n \\
0 n \\
2\end{array}$ & $\begin{array}{l}31079.93 \\
31082.23 \\
31197.55 \\
31205.12 \\
31212.12\end{array}$ & $\begin{array}{l}\mathrm{U} \\
\mathrm{Z}\end{array}$ \\
\hline $\begin{array}{l}3332.840 \\
3328.589 \\
3328.470 \\
3324.142 \\
3323.454\end{array}$ & $\begin{array}{l}0 n \\
0 \\
1 n \\
0 \\
0\end{array}$ & $\begin{array}{l}29995.81 \\
30034.12 \\
30035.19 \\
30074.30 \\
30080.52\end{array}$ & $\mathrm{U}$ & $\begin{array}{l}3202.188 \\
3200.908 \\
3200.092 \\
3198.492 \\
3195.235\end{array}$ & $\begin{array}{l}0 n \\
0 \\
1 \\
1 \\
1 n\end{array}$ & $\begin{array}{l}31219.62 \\
31232.11 \\
31240.07 \\
31255.70 \\
31287.56\end{array}$ & $\begin{array}{c}\mathrm{U} \\
\mathrm{U} \\
\mathrm{Z} \\
\mathrm{W}, \mathrm{Z}\end{array}$ \\
\hline $\begin{array}{l}3323.352 \\
3316.838 \\
3311.307 \\
3311.200 \\
3310.916\end{array}$ & $\begin{array}{l}0 n \\
0 \\
0 \\
0 n \\
0 n\end{array}$ & $\begin{array}{l}30081.45 \\
30140.52 \\
30190.87 \\
30191.84 \\
30194.43\end{array}$ & $\begin{array}{c}\mathrm{U} \\
\mathrm{U}, \mathrm{W}\end{array}$ & $\begin{array}{l}3192.521 \\
3189.612 \\
3186.276 \\
3184.215 \\
3181.142\end{array}$ & $\begin{array}{l}2 \\
0 \\
0 \\
1 \\
0 n\end{array}$ & $\begin{array}{l}31314.16 \\
31342.71 \\
31375.53 \\
31395.84 \\
31426.16\end{array}$ & $\begin{array}{l}\mathrm{Z} \\
\mathrm{U} \\
\mathrm{U} \\
\mathrm{U}\end{array}$ \\
\hline $\begin{array}{l}3309.660 \\
3309.283 \\
3305.572 \\
3305.376 \\
3305.258\end{array}$ & $\begin{array}{l}1 n \\
1 n \\
1 n \\
0 n \\
0 n\end{array}$ & $\begin{array}{l}30205.89 \\
30209.33 \\
30243.24 \\
30245.04 \\
30246.12\end{array}$ & $\begin{array}{l}\mathrm{U} \\
\mathrm{U}\end{array}$ & $\begin{array}{l}3170.978 \\
3165.280 \\
3163.494 \\
3163.372 \\
3158.193\end{array}$ & $\begin{array}{l}0 \\
0 \\
0 \\
0 \\
0\end{array}$ & $\begin{array}{l}31526.89 \\
31583.64 \\
31601.47 \\
31602.69 \\
31654.51\end{array}$ & $\begin{array}{l}\mathrm{U} \\
\mathrm{U} \\
\mathrm{Z}\end{array}$ \\
\hline $\begin{array}{l}3303.918 \\
3303.046 \\
3301.350 \\
3297.728 \\
3297.414\end{array}$ & $\begin{array}{l}0 \\
1 n \\
0 n \\
0 n \\
0\end{array}$ & $\begin{array}{l}30258.38 \\
30266.37 \\
30281.92 \\
30315.18 \\
30318.06\end{array}$ & & $\begin{array}{l}3157.293 \\
3146.915 \\
3146.270 \\
3145.431 \\
3139.485\end{array}$ & $\begin{array}{l}1 \\
0 \\
1 \\
1 \\
1\end{array}$ & $\begin{array}{l}31662.54 \\
31767.95 \\
31774.46 \\
31782.94 \\
31843.13\end{array}$ & $\begin{array}{l}\mathrm{U} \\
\mathrm{U} \\
\mathrm{U} \\
\mathrm{U}\end{array}$ \\
\hline $\begin{array}{l}3295.897 \\
3295.316 \\
3294.963 \\
3294.829 \\
3294.621\end{array}$ & $\begin{array}{l}0 \\
0 \\
0 n \\
0 \\
0 n\end{array}$ & $\begin{array}{l}30332.02 \\
30337.37 \\
30340.62 \\
30341.85 \\
30343.77\end{array}$ & $\begin{array}{l}\mathrm{U} \\
\mathrm{W} \\
\mathrm{U}\end{array}$ & $\begin{array}{l}3137.238 \\
3133.732 \\
3133.440 \\
3132.660 \\
3130.972\end{array}$ & $\begin{array}{l}0 \\
1 \\
0 \\
1 \\
0\end{array}$ & $\begin{array}{l}31865.94 \\
31901.59 \\
31904.56 \\
31912.50 \\
31929.71\end{array}$ & $\begin{array}{c}\mathrm{U} \\
\mathrm{U}^{\mathrm{e}} \\
\mathrm{U} \\
\mathrm{Z} \\
\mathrm{U}\end{array}$ \\
\hline $\begin{array}{l}\text { 3294. } 267 \\
3287.483 \\
3284.202 \\
3282.440 \\
3270.620\end{array}$ & $\begin{array}{l}0 \\
1 n \\
0 \\
1 \\
1\end{array}$ & $\begin{array}{l}30347.03 \\
30409.65 \\
30440.03 \\
30456.37 \\
30566.43\end{array}$ & $\begin{array}{c}\mathrm{U}^{\mathrm{e}} \\
\mathrm{Z}\end{array}$ & $\begin{array}{l}3129.800 \\
3125.390 \\
3119.864 \\
3115.954 \\
3109.614\end{array}$ & $\begin{array}{l}0 \\
0 \\
0 n \\
0 \\
1 n\end{array}$ & $\begin{array}{l}31941.66 \\
31986.73 \\
32043.39 \\
32083.60 \\
32149.01\end{array}$ & Z \\
\hline $\begin{array}{l}3270.312 \\
3268.885 \\
3263.989 \\
3263.805 \\
3263.062\end{array}$ & $\begin{array}{l}0 n \\
1 \\
0 \\
0 \\
1\end{array}$ & $\begin{array}{l}30569.31 \\
30582.66 \\
30628.53 \\
30630.25 \\
30637.23\end{array}$ & $\mathrm{Z}$ & $\begin{array}{l}\text { 3107. } 322 \\
3053.781 \\
3049.564 \\
\text { 3049. } 356 \\
\text { 3038. } 334\end{array}$ & $\begin{array}{l}1 n \\
0 n \\
1 n \\
1 n \\
0 n\end{array}$ & $\begin{array}{l}32172.72 \\
32736.77 \\
32782.04 \\
32784.27 \\
32903.20\end{array}$ & $\begin{array}{c}\mathrm{Z} \\
\mathrm{BK} \\
\mathrm{BK}, \mathrm{U} \\
\mathrm{BK}, \mathrm{W}, \mathrm{Z} \\
\mathrm{BK}, \mathrm{U}\end{array}$ \\
\hline
\end{tabular}


TABle 4.-New Fe I lines, unclassified-Continued

\begin{tabular}{|c|c|c|c|c|c|c|c|}
\hline $\begin{array}{c}\text { Wavelength } \\
\text { A }\end{array}$ & Intensity & $\begin{array}{l}\text { Wave Number } \\
\mathrm{cm}^{-1}\end{array}$ & $\begin{array}{l}\text { Notes and } \\
\text { References }\end{array}$ & $\begin{array}{c}\text { Wavelength } \\
\text { A }\end{array}$ & Intensity & $\begin{array}{l}\text { Wave Number } \\
\mathrm{cm}^{-1}\end{array}$ & $\begin{array}{l}\text { Notes and } \\
\text { References }\end{array}$ \\
\hline $\begin{array}{l}3012.942 \\
3010.198 \\
\text { 2995. } 256 \\
\text { 2985. } 750 \\
2979.867\end{array}$ & $\begin{array}{l}1 n \\
1 n \\
0 \\
0 \\
0\end{array}$ & $\begin{array}{l}33180.48 \\
33210.73 \\
33376.40 \\
33482.65 \\
33548.75\end{array}$ & $\begin{array}{c}\text { BK } \\
\text { BKc } \\
\text { BK, W, Z } \\
\text { BK, Z } \\
\text { BK, W, Z }\end{array}$ & $\begin{array}{l}2642.403 \\
2618.850 \\
2610.464 \\
2609.600 \\
2597.443\end{array}$ & $\begin{array}{l}0 \\
1 \\
0 \\
1 n \\
0\end{array}$ & $\begin{array}{l}37833.07 \\
38173.31 \\
38295.93 \\
38308.61 \\
38487.90\end{array}$ & $\begin{array}{c}(\mathrm{c}) \\
\text { BK } \\
\text { BK, U } \\
\text { BK } \\
\text { BK, U }\end{array}$ \\
\hline $\begin{array}{l}\text { 2975. } 298 \\
2971.776 \\
2963.518 \\
2962.585 \\
2956.377\end{array}$ & $\begin{array}{l}0 \\
0 \\
0 n \\
1 n \\
0\end{array}$ & $\begin{array}{l}33600.27 \\
33640.09 \\
33733.83 \\
33744.45 \\
33815.30\end{array}$ & $\begin{array}{l}\text { BK, U } \\
\text { BK, W, Z } \\
\text { BK, Z } \\
\text { BK, W, Z } \\
\text { BK, U }\end{array}$ & $\begin{array}{l}\text { 2591. } 782 \\
\text { 2581. } 754 \\
2569.322 \\
2568.584 \\
2563.990\end{array}$ & $\begin{array}{l}0 \\
0 n \\
1 n \\
1 \\
1\end{array}$ & $\begin{array}{l}38571.96 \\
38721.77 \\
38909.12 \\
38920.29 \\
38990.02\end{array}$ & $\begin{array}{c}\text { BK, U } \\
\text { BK, U } \\
\text { BK, Z } \\
\text { BK, U } \\
\text { BK, Uc }\end{array}$ \\
\hline $\begin{array}{l}\text { 2955. } 619 \\
\text { 2954. } 957 \\
2945.702 \\
2934.598 \\
2933.051\end{array}$ & $\begin{array}{l}0 \\
0 \\
1 \\
0 \\
1\end{array}$ & $\begin{array}{l}33823.98 \\
33831.55 \\
33937.84 \\
34066.25 \\
34084.22\end{array}$ & $\begin{array}{c}\text { BK, U } \\
\text { BK, U } \\
\text { BK, W, Z } \\
\text { BK, U } \\
\text { BK }\end{array}$ & $\begin{array}{l}2561.068 \\
2559.080 \\
2558.118 \\
2557.982 \\
2557.792\end{array}$ & $\begin{array}{l}2 \\
0 \\
1 \\
1 \\
1 n\end{array}$ & $\begin{array}{l}39034.51 \\
39064.83 \\
39079.52 \\
39081.60 \\
39084.50\end{array}$ & $\begin{array}{l}\text { BK, U } \\
\text { BK, U } \\
\text { BK, U } \\
\text { BK, U }\end{array}$ \\
\hline $\begin{array}{l}2930.395 \\
2915.658 \\
2896.772 \\
2880.398 \\
2877.724\end{array}$ & $\begin{array}{l}1 \\
0 \\
0 \\
0 \\
0\end{array}$ & $\begin{array}{l}34115.11 \\
34287.54 \\
34511.07 \\
34707.24 \\
34739.49\end{array}$ & $\begin{array}{c}\text { BK, U } \\
\text { BK, U } \\
\text { BK } \\
\text { BK, U } \\
\text { BK }\end{array}$ & $\begin{array}{l}\text { 2557. } 020 \\
2554.218 \\
2532.269 \\
2531.449 \\
\text { 2527. } 262\end{array}$ & $\begin{array}{l}0 n \\
0 n \\
1 \\
1 n \\
1\end{array}$ & $\begin{array}{l}39096.30 \\
39139.18 \\
39478.41 \\
39491.20 \\
39556.62\end{array}$ & $\begin{array}{c}\mathrm{BK} \\
\mathrm{BK}, \mathrm{U} \\
\mathrm{BK}, \mathrm{U}, \mathrm{W} \\
\mathrm{BK}, \underset{\mathrm{BK}}{\mathrm{BK}} \mathrm{Z}^{\mathrm{a}, \mathrm{e}}\end{array}$ \\
\hline $\begin{array}{l}2877.582 \\
2862.080 \\
2841.260 \\
2830.090 \\
2829.485\end{array}$ & $\begin{array}{l}0 \\
0 \\
1 \\
0 n \\
0 n\end{array}$ & $\begin{array}{l}34741.21 \\
34929.37 \\
35185.31 \\
35324.17 \\
35331.72\end{array}$ & $\begin{array}{c}\text { BK } \\
\text { BK, U, W } \\
\text { BKc } \\
\text { BK, W }\end{array}$ & $\begin{array}{l}2524.603 \\
2513.246 \\
2502.503 \\
2494.797 \\
2492.236\end{array}$ & $\begin{array}{l}1 \\
1 \\
3 \\
1 n \\
1 n\end{array}$ & $\begin{array}{l}\text { 39598. } 28 \\
39777.20 \\
39947.95 \\
40071.34 \\
40112.51\end{array}$ & $\begin{array}{l}\text { BK } \\
\text { BK, U } \\
\text { BK, U } \\
\text { BK }\end{array}$ \\
\hline $\begin{array}{l}2829.312 \\
2808.665 \\
2801.922 \\
2785.002 \\
2783.845\end{array}$ & $\begin{array}{l}0 n \\
1 \\
0 \\
1 n \\
0\end{array}$ & $\begin{array}{l}\text { 35333. } 89 \\
35593.62 \\
35679.27 \\
35896.03 \\
35910.94\end{array}$ & $\begin{array}{l}\text { BK, U } \\
\text { BK, W, Z } \\
\text { BK, U } \\
\text { BK } \\
\text { BK }\end{array}$ & $\begin{array}{l}2485.435 \\
2485.206 \\
2480.732 \\
2478.300 \\
2477.304\end{array}$ & $\begin{array}{l}0 \\
1 n \\
0 n \\
1 n \\
1 n\end{array}$ & $\begin{array}{l}\text { 40222. } 26 \\
\text { 40225. } 97 \\
\text { 40298. } 51 \\
\text { 40338. } 06 \\
\text { 40354. } 27\end{array}$ & $\begin{array}{c}\text { BK, W } \\
\text { BK } \\
\text { BK } \\
\text { BK } \\
\text { BK }^{\mathrm{c}}\end{array}$ \\
\hline $\begin{array}{l}2775.818 \\
2765.254 \\
2764.128 \\
2762.939 \\
2758.093\end{array}$ & $\begin{array}{l}1 n \\
1 \\
1 n \\
0 \\
0\end{array}$ & $\begin{array}{l}36014.79 \\
36152.36 \\
36167.09 \\
36182.65 \\
36246.22\end{array}$ & $\begin{array}{c}\text { BK, U, W } \\
\text { BK, U, Wc } \\
\text { BK, Uc } \\
\text { BK, U } \\
\text { BK, U }\end{array}$ & $\begin{array}{l}2476.464 \\
2475.758 \\
2475.466 \\
2475.018 \\
2467.953\end{array}$ & $\begin{array}{l}1 n \\
1 \\
2 \\
1 n \\
1 n\end{array}$ & $\begin{array}{l}\text { 40367. } 96 \\
40379.47 \\
40384.23 \\
40391.54 \\
40507.16\end{array}$ & $\begin{array}{l}\text { BK, U } \\
\text { BK } \\
\text { BK } \\
\text { BK, U } \\
\text { BK, U }\end{array}$ \\
\hline $\begin{array}{l}2757.558 \\
2751.325 \\
2731.883 \\
2730.265 \\
2723.892\end{array}$ & $\begin{array}{l}0 \\
1 n \\
0 \\
0 \\
1 n\end{array}$ & $\begin{array}{l}36253.26 \\
36335.38 \\
36593.96 \\
36615.64 \\
36701.30\end{array}$ & $\begin{array}{c}\mathrm{BK}, \mathrm{U} \\
\mathrm{BK}, \mathrm{Z} ? \\
\mathrm{BK}^{\mathrm{c}} \\
\mathrm{BK} \\
(\mathrm{c})\end{array}$ & $\begin{array}{l}2467.573 \\
2466.348 \\
2466.093 \\
2464.349 \\
2462.963\end{array}$ & $\begin{array}{l}2 n \\
1 n \\
0 \\
1 \\
1\end{array}$ & $\begin{array}{l}\text { 40513. } 40 \\
40533.52 \\
40537.71 \\
40566.40 \\
40589.23\end{array}$ & $\begin{array}{l}\text { BK, U } \\
\text { BK, U } \\
\text { BK, U } \\
\text { BK, U } \\
\text { BK, U }\end{array}$ \\
\hline $\begin{array}{l}2715.503 \\
2713.510 \\
2704.798 \\
2702.622 \\
2685.140\end{array}$ & $\begin{array}{l}0 \\
0 n \\
0 \\
0 n \\
1\end{array}$ & $\begin{array}{l}36814.68 \\
36841.72 \\
36960.38 \\
36990.13 \\
37230.95\end{array}$ & $\begin{array}{c}\mathrm{BK}^{\mathrm{c}} \\
\mathrm{BK}, \mathrm{U}^{\mathrm{c}} \\
\mathrm{BK}, \mathrm{U} \\
\mathrm{BK} \\
\mathrm{BK}, \mathrm{U}, \mathrm{W}^{\mathrm{c}}\end{array}$ & $\begin{array}{l}\text { 2456. } 147 \\
2454.399 \\
2452.965 \\
\text { 2451. } 697 \\
2448.826\end{array}$ & $\begin{array}{l}2 n \\
1 \\
1 \\
3 n \\
1 n\end{array}$ & $\begin{array}{l}40701.86 \\
40730.84 \\
40754.65 \\
40775.73 \\
40823.53\end{array}$ & $\begin{array}{c}\text { BK, U } \\
\text { BK, U } \\
\text { BK } \\
\text { BK, W } \\
\text { BK, U }\end{array}$ \\
\hline $\begin{array}{l}2684.536 \\
2676.162 \\
2674.625 \\
2673.552 \\
2672.831\end{array}$ & $\begin{array}{l}0 \\
1 n \\
1 \\
0 \\
0 n\end{array}$ & $\begin{array}{l}37239.33 \\
37355.84 \\
37377.31 \\
37392.31 \\
37402.40\end{array}$ & $\begin{array}{l}\text { BK, U } \\
\text { BK } \\
\text { BK, U } \\
\text { BK } \\
\text { BK }\end{array}$ & $\begin{array}{l}\text { 2440. } 335 \\
2436.072 \\
2419.236 \\
2413.764 \\
2411.738\end{array}$ & $\begin{array}{l}2 \\
2 \\
1 \\
1 \\
1 n\end{array}$ & $\begin{array}{l}\text { 40965. } 56 \\
41037.24 \\
41322.81 \\
41416.48 \\
41451.27\end{array}$ & $\begin{array}{l}\text { BK, U } \\
\text { BK, U } \\
\text { BK, U } \\
\text { BK, U } \\
\text { BK }\end{array}$ \\
\hline $\begin{array}{l}\text { 2668. } 904 \\
\text { 2668. } 724 \\
2665.814 \\
\text { 2664. } 184 \\
\text { 2663. } 169\end{array}$ & $\begin{array}{l}1 n \\
1 \\
0 \\
1 n \\
0\end{array}$ & $\begin{array}{l}37457.43 \\
37459.95 \\
37500.84 \\
37523.78 \\
37538.08\end{array}$ & $\begin{array}{c}\mathrm{BK}, \mathrm{U}, \mathrm{Wc}^{\mathrm{c}} \\
\mathrm{BK} \\
\mathrm{BK}, \mathrm{U}, \mathrm{W} \\
\mathrm{BK} \mathrm{c} \\
\mathrm{BK}, \mathrm{U}\end{array}$ & $\begin{array}{l}2405.302 \\
2403.542 \\
2402.938 \\
2402.109 \\
2398.726\end{array}$ & $\begin{array}{l}0 \\
0 n \\
1 \\
1 \\
1\end{array}$ & $\begin{array}{l}41562.18 \\
41592.61 \\
41603.06 \\
41617.42 \\
41676.11\end{array}$ & $\begin{array}{c}\mathrm{BK}, \mathrm{U} \\
\mathrm{BK}, \mathrm{U} \\
\mathrm{BK}, \mathrm{U} \\
(\mathrm{c}) \\
\mathrm{BK}\end{array}$ \\
\hline $\begin{array}{l}\text { 2658. } 699 \\
\text { 2658. } 111 \\
\text { 2656. } 337 \\
2652.212 \\
\text { 2648. } 914\end{array}$ & $\begin{array}{l}1 \\
0 \\
0 \\
0 \\
0\end{array}$ & $\begin{array}{l}37601.19 \\
37609.51 \\
37634.63 \\
37693.16 \\
37740.08\end{array}$ & $\begin{array}{c}\mathrm{BK}, \mathrm{U} \\
\mathrm{BK} \\
\mathrm{BK}, \mathrm{U} \\
\mathrm{BK}, \mathrm{U} \\
\mathrm{BK}\end{array}$ & $\begin{array}{l}2396.102 \\
2395.186 \\
2394.303 \\
2388.090 \\
2385.386\end{array}$ & $\begin{array}{l}0 n \\
1 \\
0 n \\
1 \\
1\end{array}$ & $\begin{array}{l}\text { 41721. } 74 \\
41737.70 \\
41753.09 \\
41861.71 \\
41909.16\end{array}$ & $\begin{array}{c}\mathrm{BK}, \mathrm{U}^{\mathrm{e}} \\
\mathrm{BK}, \mathrm{U} \\
\mathrm{BK} \\
\text { BK } \\
\mathrm{BK}, \mathrm{U}\end{array}$ \\
\hline
\end{tabular}


TABLe 4.-New Fe I lines, unclassified-Concluded

\begin{tabular}{|c|c|c|c|c|c|c|c|}
\hline $\begin{array}{c}\text { Wavelength } \\
\text { A }\end{array}$ & Intensity & $\begin{array}{l}\text { Wave Number } \\
\mathrm{cm}^{-1}\end{array}$ & $\begin{array}{l}\text { Notes and } \\
\text { References }\end{array}$ & $\begin{array}{c}\text { Wavelength } \\
\text { A }\end{array}$ & Intensity & $\begin{array}{l}\text { Wave Number } \\
\mathrm{cm}^{-1}\end{array}$ & $\begin{array}{l}\text { Notes and } \\
\text { References }\end{array}$ \\
\hline $\begin{array}{l}\text { 2383. } 790 \\
2376.276 \\
2375.990 \\
2360.755 \\
2358.622\end{array}$ & $\begin{array}{l}1 n \\
0 \\
0 \\
2 \\
2\end{array}$ & $\begin{array}{l}41937.22 \\
42069.81 \\
42074.88 \\
42346.38 \\
42384.68\end{array}$ & $\begin{array}{l}\text { BK, U } \\
\text { BK } \\
\text { BK } \\
\text { BK, U } \\
\text { BK }\end{array}$ & $\begin{array}{l}\text { 2300. } 242 \\
2274.288 \\
2223.747 \\
2213.828 \\
2208.280\end{array}$ & $\begin{array}{l}3 \\
1 \\
1 \\
3 \\
0\end{array}$ & $\begin{array}{l}43460.30 \\
43956.22 \\
44955.16 \\
45156.56 \\
45270.00\end{array}$ & $\begin{array}{c}\text { BK } \\
\text { BK } \\
\text { BK } \\
\text { U } \\
\text { BK, U }\end{array}$ \\
\hline $\begin{array}{l}\text { 2341. } 373 \\
\text { 2335. } 246 \\
2333.024 \\
2329.093 \\
\text { 2326. } 047\end{array}$ & $\begin{array}{l}1 \\
1 \\
1 \\
0 \\
0\end{array}$ & $\begin{array}{l}42696.90 \\
42808.91 \\
42849.68 \\
42922.00 \\
42978.20\end{array}$ & $\begin{array}{l}\text { BK, U } \\
\text { BK, U } \\
\text { BK } \\
\text { BK } \\
\text { BK, U }\end{array}$ & $\begin{array}{l}2206.444 \\
2200.494 \\
2200.084 \\
2189.514 \\
2186.760\end{array}$ & $\begin{array}{l}1 \\
3 \\
1 \\
1 \\
2\end{array}$ & $\begin{array}{l}45307.66 \\
45430.16 \\
45438.62 \\
45657.96 \\
45715.45\end{array}$ & $\begin{array}{l}\text { BK, U } \\
\text { BK, U } \\
\text { U } \\
\text { BK, } U \\
\text { BK }\end{array}$ \\
\hline $\begin{array}{l}\text { 2324. } 970 \\
2324.260 \\
\text { 2323. } 416 \\
\text { 2323. } 286 \\
\text { 2322. } 684\end{array}$ & $\begin{array}{l}0 \\
0 \\
1 \\
0 \\
0\end{array}$ & $\begin{array}{l}\text { 42998. } 11 \\
\text { 43011. } 24 \\
\text { 43026. } 86 \\
\text { 43029. } 27 \\
43040.42\end{array}$ & $\begin{array}{l}\text { BK, U } \\
\text { BK } \\
\text { BK, U } \\
\text { BK, U } \\
\text { BK }\end{array}$ & $\begin{array}{l}2176.670 \\
2176.216 \\
2175.825 \\
2173.451 \\
2172.658\end{array}$ & $\begin{array}{l}3 \\
1 \\
3 \\
1 \\
3 n\end{array}$ & $\begin{array}{l}45927.35 \\
45936.93 \\
45945.18 \\
45995.36 \\
46012.15\end{array}$ & $\begin{array}{l}\text { BK, U } \\
\text { BK, U } \\
\text { BK, X } \\
\text { BK } \\
\text { BK }\end{array}$ \\
\hline $\begin{array}{l}\text { 2321. } 583 \\
2321.220 \\
2321.029 \\
2319.680 \\
2319.441\end{array}$ & $\begin{array}{l}0 \\
0 \\
0 \\
1 n \\
0\end{array}$ & $\begin{array}{l}43060.83 \\
43067.57 \\
43071.11 \\
43096.16 \\
43100.60\end{array}$ & $\begin{array}{l}\text { BK, U } \\
\text { BK, Ue } \\
\text { BK } \\
\text { BK, U } \\
\text { BK }\end{array}$ & $\begin{array}{l}\text { 2172. } 037 \\
2156.432 \\
2151.005 \\
2146.806 \\
2146.458\end{array}$ & $\begin{array}{l}1 \\
3 \\
2 \\
3 \\
1\end{array}$ & $\begin{array}{l}\text { 46025. } 30 \\
46358.32 \\
46475.28 \\
46566.17 \\
46573.72\end{array}$ & $\begin{array}{c}\text { BK } \\
\mathrm{X}, Z^{\mathrm{d}} \\
\mathrm{BK} \\
\mathrm{BK}, \mathrm{U} \\
\mathrm{BK}\end{array}$ \\
\hline $\begin{array}{l}2316.290 \\
2314.337 \\
2313.650 \\
2310.732 \\
2304.544\end{array}$ & $\begin{array}{l}0 \\
0 \\
1 \\
0 \\
1\end{array}$ & $\begin{array}{l}\text { 43159. } 22 \\
43195.64 \\
43208.47 \\
43263.02 \\
43378.99\end{array}$ & $\begin{array}{l}\text { BK } \\
\text { BK } \\
\text { BK, U } \\
\text { BK, Uc } \\
\text { BK, U }\end{array}$ & $\begin{array}{l}2143.369 \\
2142.820 \\
2142.575 \\
2135.300 \\
2132.526 \\
\\
2124.948 \\
2121.864\end{array}$ & $\begin{array}{l}2 \\
1 \\
1 \\
0 n \\
0 n \\
2 n \\
1 n\end{array}$ & $\begin{array}{l}\text { 46640. } 83 \\
46652.78 \\
46658.11 \\
\text { 46817. } 06 \\
46877.95 \\
\\
\text { 47045. } 11 \\
47113.48\end{array}$ & $\begin{array}{l}\text { BK, U } \\
\text { BK, U } \\
\text { BK, U } \\
\text { BK } \\
\text { BK, U } \\
\text { BK, U } \\
\text { BK, U }\end{array}$ \\
\hline
\end{tabular}

TABLE 5.-Faint lines of Fe I in the solar spectrum

\begin{tabular}{|c|c|c|c|c|c|c|c|c|c|c|c|}
\hline \multicolumn{2}{|c|}{ Laboratory } & \multicolumn{4}{|c|}{ Sun } & \multicolumn{2}{|c|}{ Laboratory } & \multicolumn{4}{|c|}{ Sun } \\
\hline $\begin{array}{l}\text { Wave- } \\
\text { length } \\
A\end{array}$ & $\begin{array}{c}\text { Inten- } \\
\text { sity }\end{array}$ & $\begin{array}{c}\text { Wave- } \\
\text { length } \\
\text { A }\end{array}$ & $\begin{array}{l}\text { Inten- } \\
\text { sity } \\
\Delta \lambda / \lambda\end{array}$ & $\odot{ }_{\mathrm{A}}$ & $\begin{array}{l}\text { Solar } \\
\text { Identifi- } \\
\text { cation }\end{array}$ & $\begin{array}{l}\text { Wave- } \\
\text { length } \\
\text { A }\end{array}$ & $\begin{array}{c}\text { Inten- } \\
\text { sity }\end{array}$ & $\begin{array}{l}\text { Wave- } \\
\text { length } \\
\text { A }\end{array}$ & $\begin{array}{l}\text { Inten- } \\
\text { sity } \\
\Delta \lambda / \lambda\end{array}$ & $\odot-$ Lab. & $\begin{array}{l}\text { Solar } \\
\text { Identifi- } \\
\text { cation }\end{array}$ \\
\hline $\begin{array}{l}8126.520 \\
8090.341 \\
7714.603 \\
7588.834 \\
7438.336\end{array}$ & $\begin{array}{l}1 \\
1 \\
0 \\
0 \\
1\end{array}$ & $\begin{array}{l}8126.48 \\
8090.464 \\
7714.59 \\
7588.849 \\
7438.38\end{array}$ & $\begin{array}{l}0.6 \\
3.7 \\
1.2 \\
1.2 \\
0.2\end{array}$ & $\begin{array}{l}-0.04 \\
+0.123 \\
-0.01 \\
+0.015 \\
+0.04\end{array}$ & $\begin{array}{c}\text { Fe I? } \\
\text { Atm Fe I? } \\
\text { Fe I? } \\
\text { Fe I? } \\
\text { Fe I? }\end{array}$ & $\begin{array}{l}5173.498 \\
5149.492 \\
5147.107 \\
5140.826 \\
5097.498\end{array}$ & $\begin{array}{l}3 \\
1 \\
2 \\
3 \\
3\end{array}$ & $\begin{array}{l}5173.487 \\
5149.520 \\
5147.103 \\
5140.823 \\
5097.492\end{array}$ & $\begin{array}{l}0.9 \\
1.1 \\
\text { 5. } \\
\text { 3. } 1 \\
7.4\end{array}$ & $\begin{array}{l}-0.011 \\
+0.028 \\
-0.004 \\
-0.003 \\
-0.006\end{array}$ & $\begin{array}{c}\mathrm{Fe} \text { I } \\
\text { Fe I-Fe II } \\
\text { Fe I-Fe II } \\
\text { Fe I } \\
\text { Fe I }\end{array}$ \\
\hline $\begin{array}{l}7214.630 \\
7192.458 \\
7092.866 \\
6692.280 \\
5899.094\end{array}$ & $\begin{array}{l}1 \\
1 n \\
1 n \\
1 \\
2\end{array}$ & $\begin{array}{l}7214.60 \\
7192.465 \\
7092.848 \\
6692.304 \\
5899.106\end{array}$ & $\begin{array}{l}0.1 \\
4.4 \\
1.1 \\
0.3 \\
0.1\end{array}$ & $\begin{array}{l}-0.03 \\
+0.007 \\
-0.018 \\
+0.024 \\
+0.012\end{array}$ & $\begin{array}{c}\text { Atm? Fe I? } \\
\text { Fe I-| Atm } \\
\text { Fe I } \\
\text { Fe I? } \\
\text { Fe I }\end{array}$ & $\begin{array}{l}5031.180 \\
5025.306 \\
5013.914 \\
5007.710 \\
4992.502\end{array}$ & $\begin{array}{l}1 \\
3 \\
3 \\
3 \\
1\end{array}$ & $\begin{array}{l}5031.182 \\
5025.305 \\
5013.920 \\
5007.734 \\
4992.480\end{array}$ & $\begin{array}{l}\text { 2. } 2 \\
\text { 3. } 6 \\
\text { 4. } 4 \\
6.6 \\
0.9\end{array}$ & $\begin{array}{l}+0.002 \\
-0.001 \\
+0.006 \\
+0.024 \\
-0.022\end{array}$ & $\begin{array}{l}\text { Fe I? } \\
\text { Fe I } \\
\text { Fe I } \\
\text { Fe I? } \\
\text { Fe I? }\end{array}$ \\
\hline $\begin{array}{l}5699.308 \\
5644.033 \\
5625.704 \\
5589.852 \\
5458.572\end{array}$ & $\begin{array}{l}2 \\
3 \\
3 \\
2 n \\
2\end{array}$ & $\begin{array}{l}5699.322 \\
5644.037 \\
5625.687 \\
5589.861 \\
5458.58\end{array}$ & $\begin{array}{l}1.2 \\
2.5 \\
5.2 \\
2.9 \\
1.1\end{array}$ & $\begin{array}{l}+0.014 \\
+0.004 \\
-0.017 \\
+0.008 \\
+0.01\end{array}$ & $\begin{array}{r}\text { Fe I } \\
\text { Fe I } \\
\text {-Fe I } \\
\text { Fe I } \\
\text { Fe I }\end{array}$ & $\begin{array}{l}4980.278 \\
4974.246 \\
4961.040 \\
4944.306 \\
4902.368\end{array}$ & $\begin{array}{l}3 \\
0 \\
3 \\
3 \\
1\end{array}$ & $\begin{array}{l}4980.296 \\
4974.247 \\
4961.054 \\
4944.287 \\
4902.384\end{array}$ & $\begin{array}{l}2.8 \\
1.6 \\
\text { 3. } 4 \\
2.0 \\
1.7\end{array}$ & $\begin{array}{l}+0.018 \\
+0.001 \\
+0.014 \\
-0.019 \\
+0.016\end{array}$ & $\begin{array}{l}\text { Fe I } \\
\text { Fe I } \\
\text { Fe I } \\
\text { Fe I } \\
\text { Fe I }\end{array}$ \\
\hline $\begin{array}{l}5350.798 \\
5350.434 \\
5308.228 \\
5303.874 \\
5181.335\end{array}$ & $\begin{array}{l}1 \\
0 \\
0 \\
1 n \\
3\end{array}$ & $\begin{array}{l}5350.789 \\
5350.454 \\
5308.212 \\
5303.845 \\
5181.330\end{array}$ & $\begin{array}{l}0.7 \\
0.2 \\
0.1 \\
\text { 1. } 3 \\
\text { 3. } 7\end{array}$ & $\begin{array}{r}-0.009 \\
+0.020 \\
-0.016 \\
-0.029 \\
-0.005\end{array}$ & $\begin{array}{l}\text { Fe I } \\
\text { Fe I? } \\
\text { Fe I? } \\
\text { Fe I? } \\
\text { Fe I? }\end{array}$ & $\begin{array}{l}4900.816 \\
4861.947 \\
4833.817 \\
4825.357 \\
4821.572\end{array}$ & $\begin{array}{l}2 \\
3 \\
1 \\
3 \\
2\end{array}$ & $\begin{array}{l}4900.821 \\
4861.952 \\
4833.819 \\
4825.349 \\
4821.601\end{array}$ & $\begin{array}{l}2.4 \\
4.1 \\
\text { 1. } 7 \\
\text { 6. } 2 \\
0.8\end{array}$ & $\begin{array}{l}+0.005 \\
+0.005 \\
+0.002 \\
+0.008 \\
+0.029\end{array}$ & $\begin{array}{l}\text { Fe I } \\
\text { Fe I } \\
\text { Fe I } \\
\text { Fe I } \\
\text { Fe I }\end{array}$ \\
\hline
\end{tabular}


TABLE 5.-Faint lines of Fe I in the solar spectrum-Continued

\begin{tabular}{|c|c|c|c|c|c|c|c|c|c|c|c|}
\hline \multicolumn{2}{|c|}{ Laboratory } & \multicolumn{4}{|c|}{ Sun } & \multicolumn{2}{|c|}{ Laboratory } & \multicolumn{4}{|c|}{ Sun } \\
\hline $\begin{array}{l}\text { Wave- } \\
\text { length } \\
\text { A }\end{array}$ & $\begin{array}{c}\text { Inten- } \\
\text { sity }\end{array}$ & $\begin{array}{c}\text { Wave- } \\
\text { length } \\
\text { A }\end{array}$ & $\begin{array}{l}\text { Inten- } \\
\text { sity } \\
\Delta \lambda / \lambda\end{array}$ & $\odot \underset{\mathrm{A}}{\mathrm{L}}$ & $\begin{array}{c}\text { Solar } \\
\text { Identifi- } \\
\text { cation }\end{array}$ & $\begin{array}{c}\text { Wave- } \\
\text { length } \\
\text { A }\end{array}$ & $\begin{array}{c}\text { Inten- } \\
\text { sity }\end{array}$ & $\begin{array}{l}\text { Wave- } \\
\text { length } \\
\text { A }\end{array}$ & $\begin{array}{c}\text { Inten- } \\
\text { sity } \\
\Delta \lambda / \lambda\end{array}$ & $\odot-$ Lab. & $\begin{array}{l}\text { Solar } \\
\text { Identifi- } \\
\text { cation }\end{array}$ \\
\hline $\begin{array}{l}4818.038 \\
4814.365 \\
4805.529 \\
4768.697 \\
4756.356\end{array}$ & $\begin{array}{l}1 \\
2 \\
0 n \\
3 \\
1\end{array}$ & $\begin{array}{l}4818.032 \\
4814.369 \\
4805.55 \\
4768.700 \\
4756.366\end{array}$ & $\begin{array}{l}3.9 \\
2.7 \\
0.8 \\
5.2 \\
2.3\end{array}$ & $\begin{array}{l}-0.006 \\
+0.004 \\
+0.02 \\
+0.003 \\
+0.010\end{array}$ & $\begin{array}{l}\text { Fe I } \\
\text { Fe I } \\
\text { Fe I? } \\
\text { Fe I } \\
\text { Fe I }\end{array}$ & $\begin{array}{l}4229.406 \\
4223.221 \\
4219.018 \\
4199.557 \\
4197.512\end{array}$ & $\begin{array}{l}1 \\
1 \\
1 \\
1 n \\
0\end{array}$ & $\begin{array}{l}4229.408 \\
4223.236 \\
4219.016 \\
4199.524 \\
4197.508\end{array}$ & $\begin{array}{l}\text { 7. } 1 \\
5.2 \\
0.4 \\
0.8 \\
0.6\end{array}$ & $\begin{array}{r}+0.002 \\
+0.015 \\
-0.002 \\
-0.033 \\
-0.004\end{array}$ & $\begin{array}{l}\text { Fe I } \\
\text { Fe I } \\
\text { Fe I } \\
\text { Fe I } \\
\text { Fe I }\end{array}$ \\
\hline $\begin{array}{l}4728.160 \\
4716.483 \\
4712.515 \\
4686.641 \\
4686.348\end{array}$ & $\begin{array}{l}1 \\
0 \\
1 \\
0 \\
0 n\end{array}$ & $\begin{array}{l}4728.167 \\
4716.508 \\
4712.497 \\
4686.630 \\
4686.370\end{array}$ & $\begin{array}{l}\text { 3. } 8 \\
0.4 \\
2.8 \\
0.3 \\
1.1\end{array}$ & $\begin{array}{r}+0.007 \\
+0.025 \\
-0.018 \\
-0.011 \\
+0.022\end{array}$ & $\begin{array}{l}\text { Fe I } \\
\text { Fe I? } \\
\text { Fe I } \\
\text { Fe I? } \\
\text { Fe I? }\end{array}$ & $\begin{array}{l}4194.099 \\
4193.600 \\
4192.374 \\
4188.729 \\
4188.300\end{array}$ & $\begin{array}{l}1 \\
1 \\
1 n \\
2 n \\
1 n\end{array}$ & $\begin{array}{l}\text { 4194. } 089 \\
4193.621 \\
4192.400 \\
4188.737 \\
4188.315\end{array}$ & $\begin{array}{r}0.5 \\
3.3 \\
2.4 \\
28.6 \\
3.8\end{array}$ & $\begin{array}{l}-0.010 \\
+0.021 \\
+0.026 \\
+0.008 \\
+0.015\end{array}$ & $\begin{array}{c}\text { Fe I } \\
\text { Fe I } \\
\text { CN Fe I } \\
\text { Fe I- } \\
\text { Fe I CN? }\end{array}$ \\
\hline $\begin{array}{l}4674.297 \\
4660.920 \\
4640.958 \\
4627.532 \\
4605.610\end{array}$ & $\begin{array}{l}2 \\
1 n \\
1 \\
2 \\
2 n\end{array}$ & $\begin{array}{l}4674.303 \\
4660.907 \\
4640.973 \\
4627.549 \\
4605.594\end{array}$ & $\begin{array}{l}\text { 2. } 8 \\
\text { 4. } 1 \\
\text { 3. } 2 \\
\text { 2. } 6 \\
\text { 8. } 6\end{array}$ & $\begin{array}{l}+0.006 \\
-0.013 \\
+0.015 \\
+0.017 \\
-0.016\end{array}$ & $\begin{array}{l}\text { Fe I } \\
\text { Fe I? } \\
\text { Fe I? } \\
\text { Fe I } \\
\text { Fe I }\end{array}$ & $\begin{array}{l}4185.793 \\
4179.689 \\
4174.208 \\
4160.333 \\
4158.366\end{array}$ & $\begin{array}{l}3 \\
1 \\
0 n \\
1 n \\
1\end{array}$ & $\begin{array}{l}4185.779 \\
4179.674 \\
4174.183 \\
4160.368 \\
4158.376\end{array}$ & $\begin{array}{r}4.5 \\
0.6 \\
0.5 \\
15.4 \\
6.0\end{array}$ & $\begin{array}{l}-0.014 \\
-0.015 \\
-0.025 \\
+0.035 \\
+0.010\end{array}$ & $\begin{array}{l}\text { Fe I } \\
\text { Fe I } \\
\text { Fe I } \\
\text { Fe I- } \\
\text { Fe I }\end{array}$ \\
\hline $\begin{array}{l}4597.403 \\
4591.502 \\
4590.815 \\
4585.337 \\
4582.297\end{array}$ & $\begin{array}{l}2 \\
2 n \\
2 n \\
1 \\
1 n\end{array}$ & $\begin{array}{l}4597.383 \\
4591.520 \\
4590.793 \\
4585.343 \\
4582.309\end{array}$ & $\begin{array}{l}5.0 \\
7.4 \\
5.4 \\
3.0 \\
3.5\end{array}$ & $\begin{array}{r}-0.020 \\
+0.018 \\
-0.022 \\
+0.006 \\
+0.012\end{array}$ & $\begin{array}{l}\text { Fe I } \\
\text { Fe I } \\
\text { Fe I } \\
\text { Fe I } \\
\text { Fe I }\end{array}$ & $\begin{array}{l}4156.322 \\
4155.914 \\
4148.794 \\
4139.718 \\
4137.642\end{array}$ & $\begin{array}{l}1 n \\
1 \\
1 n \\
1 \\
1\end{array}$ & $\begin{array}{l}\text { 4156. } 307 \\
4155.915 \\
4148.783 \\
4139.732 \\
4137.655\end{array}$ & $\begin{array}{r}20.4 \\
8.4 \\
4.8 \\
0.8 \\
8.0\end{array}$ & $\begin{array}{r}-0.015 \\
+0.001 \\
-0.011 \\
+0.014 \\
+0.013\end{array}$ & $\begin{array}{c}\text { Fe I } \\
\text { Fe I } \\
\text { Fe I Mn I } \\
\text { Fe I } \\
\text { Ce II Fe I }\end{array}$ \\
\hline $\begin{array}{l}4581.186 \\
4561.426 \\
4560.892 \\
4557.287 \\
4541.319\end{array}$ & $\begin{array}{l}2 \\
2\end{array}$ & $\begin{array}{l}4581.196 \\
4561.417 \\
4560.869 \\
4557.284 \\
4541.318\end{array}$ & $\begin{array}{l}\text { 4. } 8 \\
6.1 \\
\text { 3. } 1 \\
\text { 5. } 7 \\
\text { 3. } 7\end{array}$ & $\begin{array}{r}+0.010 \\
-0.009 \\
-0.023 \\
-0.003 \\
-0.001\end{array}$ & $\begin{array}{l}\text { Fe I } \\
\text { Fe I } \\
\text { Fe I? } \\
\text { Fe I } \\
\text { Fe I }\end{array}$ & $\begin{array}{l}4135.039 \\
4131.146 \\
4124.490 \\
4124.332 \\
4110.310\end{array}$ & $\begin{array}{l}1 n \\
1 n \\
1 \\
0 n \\
1\end{array}$ & $\begin{array}{l}4135.037 \\
4131.117 \\
4124.489 \\
4124.358 \\
4110.299\end{array}$ & $\begin{array}{r}9.2 \\
11.9 \\
7.5 \\
0.5 \\
2.2\end{array}$ & $\begin{array}{l}-0.002 \\
-0.029 \\
-0.001 \\
+0.026 \\
-0.011\end{array}$ & $\begin{array}{c}\text { Fe I Mn I } \\
\text { Mn I Fe I } \\
\text { Fe I } \\
\text { Fe I? } \\
\text { Ca II - Fe I }\end{array}$ \\
\hline $\begin{array}{l}4533.078 \\
4517.136 \\
4507.232 \\
4500.652 \\
4480.731\end{array}$ & $\begin{array}{l}2 n \\
1 \\
1 \\
1 n \\
1 n\end{array}$ & $\begin{array}{l}4533.046 \\
4517.154 \\
4507.227 \\
4500.639 \\
4480.704\end{array}$ & $\begin{array}{l}7.5 \\
6.6 \\
1.4 \\
2.9 \\
1.0\end{array}$ & $\begin{array}{r}-0.032 \\
+0.018 \\
-0.005 \\
-0.013 \\
-0.027\end{array}$ & $\begin{array}{l}\text { Fe I? } \\
\text { Fe } 1 ? \\
\text { Fe } 1 \\
\text { Fe I } \\
\text { Fe I? }\end{array}$ & $\begin{array}{l}4096.695 \\
4095.346 \\
4094.422 \\
4081.264 \\
4072.332\end{array}$ & $\begin{array}{l}1 \\
1 \\
1 \\
1 \\
0\end{array}$ & $\begin{array}{l}4096.696 \\
4095.356 \\
4094.422 \\
4081.262 \\
4072.351\end{array}$ & $\begin{array}{r}9.0 \\
4.9 \\
12.0 \\
12.2 \\
3.7\end{array}$ & $\begin{array}{r}+0.001 \\
+0.010 \\
0.000 \\
-0.002 \\
+0.019\end{array}$ & $\begin{array}{l}\text { Fe I } \\
\text { Fe I } \\
\text { Fe I } \\
\text { Fe I } \\
\text { Fe I }\end{array}$ \\
\hline $\begin{array}{l}4478.649 \\
4445.050 \\
4437.695 \\
4424.608 \\
4424.061\end{array}$ & $\begin{array}{l}1 n \\
0 \\
1 \\
1 \\
1\end{array}$ & $\begin{array}{l}4478.626 \\
4445.065 \\
4437.699 \\
4424.586 \\
4424.072\end{array}$ & $\begin{array}{l}2.7 \\
0.4 \\
\text { 2. } 7 \\
\text { 5. } 9 \\
\text { 5. } 4\end{array}$ & $\begin{array}{l}-0.023 \\
+0.015 \\
+0.004 \\
-0.022 \\
+0.011\end{array}$ & 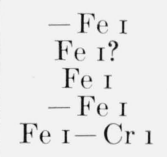 & $\begin{array}{l}4070.010 \\
4069.610 \\
4068.898 \\
4054.454 \\
4037.136\end{array}$ & $\begin{array}{l}0 \\
1 \\
0 \\
1 \\
1 n\end{array}$ & $\begin{array}{l}4070.036 \\
4069.610 \\
4068.90 \\
\text { 4054. } 442 \\
\text { 4037. } 121\end{array}$ & $\begin{array}{r}2.7 \\
8.4 \\
0.2 \\
6.9 \\
10.9\end{array}$ & $\begin{array}{r}+0.026 \\
0.000 \\
0.00 \\
-0.012 \\
-0.015\end{array}$ & $\begin{array}{l}\text { Fe I } \\
\text { Fe I } \\
\text { Fe I } \\
\text { Fe I } \\
\text { Fe I }\end{array}$ \\
\hline $\begin{array}{l}\text { 4420. } 266 \\
4419.076 \\
4416.137 \\
4412.146 \\
4411.914\end{array}$ & $\begin{array}{l}2 n \\
2 n \\
0 \\
1 \\
2 n\end{array}$ & $\begin{array}{l}4420.287 \\
4419.104 \\
4416.160 \\
4412.138 \\
4411.935\end{array}$ & $\begin{array}{r}7.7 \\
2.7 \\
1.5 \\
1.8 \\
12.0\end{array}$ & $\begin{array}{l}+0.021 \\
+0.028 \\
+0.023 \\
-0.008 \\
+0.021\end{array}$ & $\begin{array}{c}\text { Fe I } \\
\text { Cr I Fe I } \\
\text { Fe I? } \\
\text { Fe I } \\
\text { |Ti II Fe I? }\end{array}$ & $\begin{array}{l}4036.552 \\
4033.648 \\
4026.770 \\
4022.564 \\
4022.212\end{array}$ & $\begin{array}{l}0 n \\
1 \\
1 \\
0 \\
1 n\end{array}$ & $\begin{array}{l}\text { 4036. } 567 \\
4033.660 \\
4026.771 \\
4022.536 \\
4022.226\end{array}$ & $\begin{array}{r}2.1 \\
7.7 \\
2.4 \\
1.1 \\
10.4\end{array}$ & $\begin{array}{r}+0.015 \\
+0.012 \\
+0.001 \\
-0.028 \\
+0.014\end{array}$ & $\begin{array}{l}\text { Fe I } \\
\text { Fe I } \\
\text { Fe I } \\
\text { Fe I? } \\
\text { Fe I }\end{array}$ \\
\hline $\begin{array}{l}4378.486 \\
4372.558 \\
4344.928 \\
4341.802 \\
4336.122\end{array}$ & $\begin{array}{l}2 \\
1 \\
1 \\
0 n \\
1\end{array}$ & $\begin{array}{l}4378.512 \\
4372.588 \\
4344.891 \\
4341.826 \\
4336.135\end{array}$ & $\begin{array}{l}\text { 5. } 7 \\
\text { 1. } 4 \\
\text { 8. } 0 \\
2.8 \\
0.1\end{array}$ & $\begin{array}{l}+0.026 \\
+0.030 \\
+0.037 \\
+0.024 \\
+0.013\end{array}$ & $\begin{array}{l}\text { Fe I } \\
\text { Fe I } \\
\text {-Fe I? } \\
\text { Fe I } \\
\text { Fe I }\end{array}$ & $\begin{array}{l}4012.628 \\
4010.618 \\
4010.522 \\
4009.388 \\
4009.240\end{array}$ & $\begin{array}{l}1 n \\
1 \\
1 \\
1 \\
1 n\end{array}$ & $\begin{array}{l}4012.602 \\
4010.588 \\
4010.492 \\
4009.420 \\
4009.255\end{array}$ & $\begin{array}{r}6.2 \\
16.7 \\
2.7 \\
0.9 \\
1.1\end{array}$ & $\begin{array}{r}-0.026 \\
-0.030 \\
-0.030 \\
+0.032 \\
+0.015\end{array}$ & $\begin{array}{c}\mathrm{Ni} \text { I?_- Fe I } \\
\text { Fe I } \\
\text { Fe I? } \\
\text { Fe I } \\
\text { Fe I }\end{array}$ \\
\hline $\begin{array}{l}\text { 4335. } 773 \\
4334.928 \\
4332.432 \\
4331.411 \\
4288.297\end{array}$ & $\begin{array}{l}0 \\
1 n \\
1 \\
1 n \\
1\end{array}$ & $\begin{array}{l}4335.783 \\
4334.938 \\
4332.453 \\
4331.442 \\
4288.268\end{array}$ & $\begin{array}{l}\text { 1. } 3 \\
\text { 3. } 2 \\
\text { 1. } 8 \\
\text { 4. } 4 \\
\text { 1. } 2\end{array}$ & $\begin{array}{l}+0.010 \\
+0.010 \\
+0.021 \\
+0.031 \\
-0.029\end{array}$ & $\begin{array}{l}\text { Fe I?- } \\
\text { Fe I? } \\
\text { Fe I } \\
\text { Fe I- } \\
\text { Fe I? }\end{array}$ & $\begin{array}{l}\text { 4003. } 287 \\
4001.212 \\
3996.540 \\
3996.139 \\
3993.642\end{array}$ & $\begin{array}{l}1 n \\
1 N \\
1 \\
1 \\
1\end{array}$ & $\begin{array}{l}\text { 4003. } 275 \\
4001.241 \\
3996.546 \\
\text { 3996. } 117 \\
\text { 3993. } 612\end{array}$ & $\begin{array}{l}2.5 \\
1.0 \\
2.6 \\
4.0 \\
5.0\end{array}$ & $\begin{array}{r}-0.012 \\
+0.029 \\
+0.006 \\
-0.022 \\
-0.030\end{array}$ & $\begin{array}{c}\text { Mn I-Fe I } \\
\text { Fe I } \\
\text { Fe I } \\
\text { Fe I? } \\
\text { Fe I }\end{array}$ \\
\hline $\begin{array}{l}4276.082 \\
4272.528 \\
4269.730 \\
4269.053 \\
4243.560\end{array}$ & $\begin{array}{l}1 \\
1 \\
1 n \\
2 \\
2\end{array}$ & $\begin{array}{l}4276.103 \\
4272.544 \\
4269.740 \\
4269.034 \\
4243.547\end{array}$ & $\begin{array}{r}4.7 \\
10.5 \\
17.8 \\
3.5 \\
13.7\end{array}$ & $\begin{array}{r}+0.021 \\
+0.016 \\
+0.010 \\
-0.019 \\
-0.013\end{array}$ & $\begin{array}{c}\mathrm{CH} \text { Fe I } \\
\text { Fe I- } \\
\text { Fe I } \\
\text { Fe I } \\
\text { Fe I }\end{array}$ & $\begin{array}{l}3989.006 \\
3983.518 \\
3980.008 \\
3970.863 \\
3963.438\end{array}$ & $\begin{array}{l}1 N \\
1 n \\
1 \\
1 \\
1\end{array}$ & $\begin{array}{l}3988.992 \\
3983.540 \\
3980.012 \\
3970.843 \\
3963.437\end{array}$ & $\begin{array}{r}18.8 \\
10.3 \\
6.8 \\
3.3 \\
12.1\end{array}$ & $\begin{array}{r}-0.014 \\
+0.022 \\
+0.004 \\
-0.020 \\
-0.001\end{array}$ & $\begin{array}{l}\text { - Fe I } \\
\text { Fe I } \\
\text { Fe I } \\
\text { Fe I? } \\
\text { Fe I }\end{array}$ \\
\hline
\end{tabular}




\begin{tabular}{|c|c|c|c|c|c|c|c|c|c|c|c|}
\hline \multicolumn{2}{|c|}{ Laboratory } & \multicolumn{4}{|c|}{ Sun } & \multicolumn{2}{|c|}{ Laboratory } & \multicolumn{4}{|c|}{ Sun } \\
\hline $\begin{array}{l}\text { Wave- } \\
\text { length } \\
\text { A }\end{array}$ & $\begin{array}{l}\text { Inten- } \\
\text { sity }\end{array}$ & $\begin{array}{l}\text { Wave- } \\
\text { length } \\
\text { A }\end{array}$ & $\begin{array}{l}\text { Inten- } \\
\text { sity } \\
\Delta \lambda / \lambda\end{array}$ & $\odot-$ Lab. & $\begin{array}{c}\text { Solar } \\
\text { Identifi- } \\
\text { cation }\end{array}$ & $\begin{array}{c}\text { Wave- } \\
\text { length } \\
\text { A }\end{array}$ & $\begin{array}{c}\text { Inten- } \\
\text { sity }\end{array}$ & $\begin{array}{l}\text { Wave- } \\
\text { length } \\
\mathrm{A}\end{array}$ & $\begin{array}{c}\text { Inten- } \\
\text { sity } \\
\Delta \lambda / \lambda\end{array}$ & $\odot$ - Lab. & $\begin{array}{l}\text { Solar } \\
\text { Identifi- } \\
\text { cation }\end{array}$ \\
\hline $\begin{array}{l}3962.717 \\
3960.642 \\
3951.638 \\
3943.166 \\
3936.558\end{array}$ & $\begin{array}{l}1 n \\
1 \\
1 N \\
1 n \\
1\end{array}$ & $\begin{array}{l}3962.722 \\
3960.647 \\
3951.626 \\
3943.182 \\
3936.557\end{array}$ & $\begin{array}{r}12.0 \\
1.4 \\
7.1 \\
11.5 \\
16.2\end{array}$ & $\begin{array}{r}+0.005 \\
+0.005 \\
-0.012 \\
+0.016 \\
-0.001\end{array}$ & $\begin{array}{l}\text { Fe I } \\
\text { Fe I } \\
\text { Fe I } \\
\text { Fe I } \\
\text { Fe I }\end{array}$ & $\begin{array}{l}3628.414 \\
3625.498 \\
3622.431 \\
3619.942 \\
3618.160\end{array}$ & $\begin{array}{l}0 \\
1 \\
3 \\
2 n \\
2\end{array}$ & $\begin{array}{l}3628.439 \\
3625.501 \\
3622.438 \\
3619.937 \\
3618.187\end{array}$ & $\begin{array}{r}2.2 \\
13.5 \\
0.8 \\
13.8 \\
5.3\end{array}$ & $\begin{array}{r}+0.025 \\
+0.003 \\
+0.007 \\
-0.005 \\
+0.027\end{array}$ & $\begin{array}{l}\text { Fe I } \\
\text { Fe I } \\
\text { Fe I } \\
\text { Fe I } \\
\text { Fe I }\end{array}$ \\
\hline $\begin{array}{l}3934.356 \\
3932.266 \\
3931.883 \\
3930.876 \\
3892.302\end{array}$ & $\begin{array}{l}1 n \\
1 n \\
0 \\
0 N \\
1\end{array}$ & $\begin{array}{l}3934.366 \\
3932.254 \\
3931.898 \\
3930.889 \\
3892.314\end{array}$ & $\begin{array}{r}35.3 \\
5.6 \\
6.1 \\
10.4 \\
10.3\end{array}$ & $\begin{array}{r}+0.010 \\
-0.012 \\
+0.015 \\
+0.013 \\
+0.012\end{array}$ & $\begin{array}{c}\text { Fe I } \\
\text { Fe I } \\
\text { Fe I } \\
\text { Fe I- } \\
\text { Fe I }\end{array}$ & $\begin{array}{l}3617.007 \\
3616.857 \\
3616.722 \\
3615.959 \\
3615.814\end{array}$ & $\begin{array}{l}2 \\
0 \\
1 \\
1 \\
2\end{array}$ & $\begin{array}{l}3617.011 \\
3616.878 \\
3616.728 \\
3615.962 \\
3615.811\end{array}$ & $\begin{array}{r}10.6 \\
0.1 \\
1.0 \\
10.2 \\
2.5\end{array}$ & $\begin{array}{l}+0.004 \\
+0.021 \\
+0.006 \\
+0.003 \\
-0.003\end{array}$ & $\begin{array}{l}\mathrm{Fe} \text { I } \\
\mathrm{Fe} \text { I ? } \\
\mathrm{Fe} \mathrm{I} \\
\mathrm{Fe} \\
\mathrm{F} \quad \mathrm{I}\end{array}$ \\
\hline $\begin{array}{l}3818.593 \\
3815.188 \\
3814.785 \\
3805.771 \\
3804.501\end{array}$ & $\begin{array}{l}2 n \\
1 n \\
1 \\
3 \\
1\end{array}$ & $\begin{array}{l}3818.620 \\
3815.210 \\
3814.784 \\
3805.745 \\
3804.486\end{array}$ & $\begin{array}{r}21.7 \\
8.9 \\
18.9 \\
11.3 \\
7.1\end{array}$ & $\begin{array}{l}+0.027 \\
+0.022 \\
-0.001 \\
-0.026 \\
-0.015\end{array}$ & $\begin{array}{c}\text { Fe I } \\
\text { Fe } \mathrm{I}-\mathrm{CN} \\
\mathrm{Fe} I \\
\mathrm{CN}-\mathrm{Fe} I \\
\mathrm{CN}-\mathrm{Fe} \mathrm{I}\end{array}$ & $\begin{array}{l}3615.518 \\
3615.328 \\
3613.711 \\
3613.612 \\
3611.188\end{array}$ & $\begin{array}{l}0 \\
0 \\
1 \\
2 \\
1 n\end{array}$ & $\begin{array}{l}3615.531 \\
3615.324 \\
3613.719 \\
3613.605 \\
3611.184\end{array}$ & $\begin{array}{r}0.5 \\
0.8 \\
9.7 \\
24.4 \\
15.0\end{array}$ & $\begin{array}{r}+0.013 \\
-0.004 \\
+0.008 \\
-0.007 \\
-0.004\end{array}$ & $\begin{array}{l}\mathrm{Fe} \\
\mathrm{Fe} \\
\mathrm{Fe} \\
\mathrm{Fe} \\
\mathrm{Fe}\end{array}$ \\
\hline $\begin{array}{l}3801.337 \\
3799.024 \\
3794.174 \\
3793.136 \\
3786.443\end{array}$ & $\begin{array}{l}1 \\
0 \\
0 \\
1 \\
3\end{array}$ & $\begin{array}{l}3801.371 \\
3799.021 \\
3794.176 \\
3793.125 \\
3786.448\end{array}$ & $\begin{array}{r}30.2 \\
1.1 \\
1.6 \\
11.3 \\
26.6\end{array}$ & $\begin{array}{l}+0.034 \\
-0.003 \\
+0.002 \\
-0.011 \\
+0.005\end{array}$ & $\begin{array}{c}\text { Fe } 1-\mathrm{CN} \\
\text { Fe I? } \\
\text { Fe I? } \\
\text { Fe I? CN? } \\
\text { Fe I? }\end{array}$ & $\begin{array}{l}3609.996 \\
3607.780 \\
3607.256 \\
3607.102 \\
3606.253\end{array}$ & $\begin{array}{l}1 \\
2 n \\
1 \\
2 n \\
2\end{array}$ & $\begin{array}{l}3609.978 \\
3607.772 \\
3607.251 \\
3607.124 \\
3606.251\end{array}$ & $\begin{array}{l}1.2 \\
1.8 \\
0.8 \\
1.7 \\
1.4\end{array}$ & $\begin{array}{l}-0.018 \\
-0.008 \\
-0.005 \\
+0.022 \\
-0.002\end{array}$ & $\begin{array}{l}\mathrm{Fe} \text { I ? } \\
\mathrm{Fe} \text { I } \\
\mathrm{Fe} \text { I } \\
\mathrm{Fe} \text { I } \\
\mathrm{Fe} \text { I }\end{array}$ \\
\hline $\begin{array}{l}3784.812 \\
3784.698 \\
3782.862 \\
3781.300 \\
3780.966\end{array}$ & $\begin{array}{l}1 \\
1 \\
3 \\
0 \\
0\end{array}$ & $\begin{array}{l}3784.826 \\
3784.675 \\
3782.848 \\
3781.321 \\
3780.989\end{array}$ & $\begin{array}{l}2.3 \\
2.4 \\
1.2 \\
1.3 \\
2.6\end{array}$ & $\begin{array}{l}+0.014 \\
-0.023 \\
-0.014 \\
+0.021 \\
+0.023\end{array}$ & $\begin{array}{c}\text { Fe I CN } \\
\text { CN Fe I? } \\
\text { Fe I } \\
\text { Fe } 1 ? \\
\text { Fe } 1 ?-\mathrm{CN} ?\end{array}$ & $\begin{array}{l}3606.016 \\
3604.701 \\
3604.090 \\
3603.956 \\
3603.673\end{array}$ & $\begin{array}{l}0 \\
2 \\
0 n \\
2 \\
2\end{array}$ & $\begin{array}{l}\text { 3606. } 039 \\
3604.702 \\
3604.07 \\
3603.950 \\
3603.691\end{array}$ & $\begin{array}{r}7.5 \\
17.3 \\
0.5 \\
12.6 \\
9.0\end{array}$ & $\begin{array}{l}+0.023 \\
+0.001 \\
-0.02 \\
-0.006 \\
+0.018\end{array}$ & $\begin{array}{l}\text { Fe I ? } \\
\text { Fe I } \\
\text { Fe I ? } \\
\text { Fe I } \\
\text { Fe I }\end{array}$ \\
\hline $\begin{array}{l}3778.809 \\
3776.838 \\
3770.548 \\
3769.310 \\
3759.597\end{array}$ & $\begin{array}{l}1 \\
0 \\
0 \\
1 n \\
1 n\end{array}$ & $\begin{array}{l}3778.798 \\
3776.839 \\
3770.531 \\
3769.316 \\
3759.585\end{array}$ & $\begin{array}{r}23.9 \\
0.5 \\
3.7 \\
2.8 \\
13.8\end{array}$ & $\begin{array}{l}-0.011 \\
+0.001 \\
-0.017 \\
+0.006 \\
-0.012\end{array}$ & $\begin{array}{c}\text { CN Fe I? } \\
\text { Fe } 1 ? \\
\text { Fe I? } \\
\text { Fe I- } \\
\text { Fe I }\end{array}$ & $\begin{array}{l}3603.449 \\
3602.898 \\
3601.273 \\
3599.972 \\
3599.842\end{array}$ & $\begin{array}{l}1 \\
0 \\
1 \\
2 n \\
1\end{array}$ & $\begin{array}{l}3603.438 \\
3602.878 \\
3601.284 \\
3599.970 \\
3599.831\end{array}$ & $\begin{array}{r}0.9 \\
0.5 \\
1.7 \\
16.2 \\
1.8\end{array}$ & $\begin{array}{l}-0.011 \\
-0.020 \\
+0.011 \\
-0.002 \\
-0.011\end{array}$ & $\begin{array}{l}\text { Fe I } \\
\text { Fe } 1 \\
\text { Fe I } \\
\text { Fe I } \\
\text { Fe I }\end{array}$ \\
\hline $\begin{array}{l}3741.486 \\
3728.972 \\
3707.578 \\
3707.458 \\
3707.335\end{array}$ & $\begin{array}{l}1 n \\
2 \\
1 n \\
2 \\
1\end{array}$ & $\begin{array}{l}3741.479 \\
3728.954 \\
3707.562 \\
3707.465 \\
3707.329\end{array}$ & $\begin{array}{l}20.7 \\
28.1 \\
29.2 \\
25.4 \\
27.6\end{array}$ & $\begin{array}{l}-0.007 \\
-0.018 \\
-0.016 \\
+0.007 \\
-0.006\end{array}$ & $\begin{array}{l}\text { Fe I } \\
\mathrm{Ni} \text { I-Fe I } \\
\mathrm{Ti} \text { I-Fe I } \\
\text { Co I Fe I } \\
\text {-Fe I }\end{array}$ & $\begin{array}{l}3596.853 \\
3596.727 \\
3595.526 \\
3594.312 \\
3592.354\end{array}$ & $\begin{array}{l}1 n \\
0 \\
1 \\
0 \\
1\end{array}$ & $\begin{array}{l}3596.859 \\
3596.752 \\
3595.540 \\
3594.317 \\
3592.367\end{array}$ & $\begin{array}{l}0.8 \\
0.4 \\
0.3 \\
2.1 \\
1.1\end{array}$ & $\begin{array}{r}+0.006 \\
+0.025 \\
+0.014 \\
+0.005 \\
+0.013\end{array}$ & $\begin{array}{l}\text { Fe I } \\
\text { Fe I ? } \\
\text { Fe I } \\
\text { Fe i ? } \\
\text { Fe I }\end{array}$ \\
\hline $\begin{array}{l}3699.810 \\
3696.548 \\
3695.632 \\
3688.198 \\
3684.552\end{array}$ & $\begin{array}{l}1 \\
1 n \\
2 n \\
1 \\
1\end{array}$ & $\begin{array}{l}3699.825 \\
3696.523 \\
3695.652 \\
3688.173 \\
3684.542\end{array}$ & $\begin{array}{l}12.0 \\
17.6 \\
19.8 \\
19.0 \\
14.1\end{array}$ & $\begin{array}{r}+0.015 \\
-0.025 \\
+0.020 \\
-0.025 \\
-0.010\end{array}$ & $\begin{array}{l}\text { Fe I } \\
\text { Fe i } \\
\text { Fe I } \\
\text { Fe I } \\
\text { Fe I }\end{array}$ & $\begin{array}{l}3589.876 \\
3584.354 \\
3584.264 \\
3584.110 \\
3583.921\end{array}$ & $\begin{array}{l}0 n \\
1 \\
1 n \\
1 \\
1 n\end{array}$ & $\begin{array}{l}3589.882 \\
3584.383 \\
3584.257 \\
3584.097 \\
3583.911\end{array}$ & $\begin{array}{r}4.7 \\
7.9 \\
1.5 \\
10.3 \\
27.9\end{array}$ & $\begin{array}{r}+0.006 \\
+0.029 \\
-0.007 \\
-0.013 \\
-0.010\end{array}$ & $\begin{array}{l}\text { Fe I ? } \\
\text { Fe I } \\
\text { Fe I } \\
\text { Fe I } \\
\text { Fe I }\end{array}$ \\
\hline $\begin{array}{l}3680.962 \\
3680.396 \\
3677.503 \\
3672.114 \\
3665.845\end{array}$ & $\begin{array}{l}2 n \\
2 n \\
2 \\
0 \\
1\end{array}$ & $\begin{array}{l}3680.944 \\
3680.389 \\
3677.514 \\
3672.124 \\
3665.850\end{array}$ & $\begin{array}{r}23.8 \\
21.2 \\
26.4 \\
6.0 \\
6.3\end{array}$ & $\begin{array}{l}-0.018 \\
-0.007 \\
+0.011 \\
+0.010 \\
+0.005\end{array}$ & $\begin{array}{l}\text { Fe I } \\
\text { Fe I } \\
\text { Fe I } \\
\text { Fe I } \\
\text { Fe I }\end{array}$ & $\begin{array}{l}3583.687 \\
3583.577 \\
3582.970 \\
3582.908 \\
3582.460\end{array}$ & $\begin{array}{l}2 \\
1 \\
3 n \\
1 \\
2\end{array}$ & $\begin{array}{l}3583.697 \\
3583.597 \\
3582.964 \\
3582.877 \\
3582.437\end{array}$ & $\begin{array}{r}33.2 \\
3.9 \\
5.9 \\
6.6 \\
7.8\end{array}$ & $\begin{array}{l}+0.010 \\
+0.020 \\
-0.006 \\
-0.031 \\
-0.023\end{array}$ & 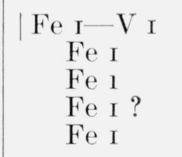 \\
\hline $\begin{array}{l}3659.214 \\
3659.094 \\
3651.918 \\
3650.554 \\
3640.096\end{array}$ & $\begin{array}{l}1 \\
1 n \\
1 n \\
2 n \\
1\end{array}$ & $\begin{array}{l}3659.234 \\
3659.124 \\
3651.921 \\
3650.538 \\
3640.118\end{array}$ & $\begin{array}{r}3.8 \\
0.7 \\
25.6 \\
25.5 \\
2.3\end{array}$ & $\begin{array}{r}+0.020 \\
+0.030 \\
+0.003 \\
-0.016 \\
+0.022\end{array}$ & $\begin{array}{l}\text { Ce II? Fe I } \\
\text { Fe I } \\
\begin{array}{c}\mathrm{Fe} \text { I } \mathrm{CH} \\
\mathrm{Fe} \mathrm{I}\end{array}\end{array}$ & $\begin{array}{l}3581.951 \\
3580.402 \\
3579.562 \\
3577.490 \\
3574.609\end{array}$ & $\begin{array}{l}1 \\
1 \\
1 \\
1 n \\
2\end{array}$ & $\begin{array}{l}3581.941 \\
3580.412 \\
3579.562 \\
3577.465 \\
3574.584\end{array}$ & $\begin{array}{r}16.4 \\
12.5 \\
9.9 \\
13.1 \\
1.5\end{array}$ & $\begin{array}{r}-0.010 \\
+0.010 \\
0.000 \\
-0.025 \\
-0.025\end{array}$ & $\begin{array}{c}\text { Fe I } \\
\text { Fe I } \\
\text { Fe I } \\
\text { Ce II- Fe I } \\
\text { Fe I }\end{array}$ \\
\hline $\begin{array}{l}3639.964 \\
3639.502 \\
3639.308 \\
3628.868 \\
3628.620\end{array}$ & $\begin{array}{l}1 n \\
1 n \\
1 n \\
1 \\
1\end{array}$ & $\begin{array}{l}3639.985 \\
3639.525 \\
3639.332 \\
3628.879 \\
3628.599\end{array}$ & $\begin{array}{r}2.6 \\
12.3 \\
3.3 \\
3.4 \\
12.7\end{array}$ & $\begin{array}{l}+0.021 \\
+0.023 \\
+0.024 \\
+0.011 \\
-0.021\end{array}$ & $\begin{array}{c}\text { Fe I } \\
\text { Fe I } \\
\text { Fe I } \\
\text { Fe I } \\
\text { Fe I }\end{array}$ & $\begin{array}{l}3574.256 \\
3567.748 \\
3562.269 \\
3555.736 \\
3539.376\end{array}$ & $\begin{array}{l}1 \\
1 \\
1 n \\
1 \\
1\end{array}$ & $\begin{array}{l}3574.253 \\
3567.742 \\
3562.270 \\
3555.724 \\
3539.371\end{array}$ & $\begin{array}{r}12.6 \\
9.5 \\
10.9 \\
3.2 \\
1.1\end{array}$ & $\begin{array}{l}-0.003 \\
-0.006 \\
+0.001 \\
-0.012 \\
-0.005\end{array}$ & 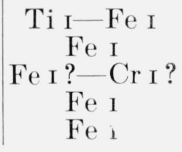 \\
\hline
\end{tabular}


TABLE 5.-Faint lines of Fe I in the solar spectrum-Continued

\begin{tabular}{|c|c|c|c|c|c|c|c|c|c|c|c|}
\hline \multicolumn{2}{|c|}{ Laboratory } & \multicolumn{4}{|c|}{ Sun } & \multicolumn{2}{|c|}{ Laboratory } & \multicolumn{4}{|c|}{ Sun } \\
\hline $\begin{array}{l}\text { Wave- } \\
\text { length } \\
\text { A }\end{array}$ & $\begin{array}{c}\text { Inten- } \\
\text { sity }\end{array}$ & $\begin{array}{l}\text { Wave- } \\
\text { length } \\
\text { A }\end{array}$ & $\begin{array}{l}\text { Inten- } \\
\text { sity } \\
\Delta \lambda / \lambda\end{array}$ & $\odot$ - Lab. & $\begin{array}{l}\text { Solar } \\
\text { Identifi- } \\
\text { cation }\end{array}$ & $\begin{array}{l}\text { Wave- } \\
\text { length } \\
\text { A }\end{array}$ & $\begin{array}{c}\text { Inten- } \\
\text { sity }\end{array}$ & $\begin{array}{l}\text { Wave- } \\
\text { length } \\
\text { A }\end{array}$ & $\begin{array}{l}\text { Inten- } \\
\text { sity } \\
\Delta \lambda / \lambda\end{array}$ & $\odot \underset{A}{-L a b .}$ & $\begin{array}{l}\text { Solar } \\
\text { Identifi- } \\
\text { cation }\end{array}$ \\
\hline $\begin{array}{l}3538.688 \\
3530.976 \\
3528.316 \\
3525.622 \\
3519.500\end{array}$ & $\begin{array}{l}1 \\
1 \\
0 \\
1 \\
1\end{array}$ & $\begin{array}{l}3538.690 \\
3530.965 \\
3528.324 \\
3525.618 \\
3519.505\end{array}$ & $\begin{array}{r}2.3 \\
7.1 \\
3.8 \\
12.6 \\
1.1\end{array}$ & $\begin{array}{l}+0.002 \\
-0.011 \\
+0.008 \\
-0.004 \\
+0.005\end{array}$ & $\begin{array}{c}\text { Fe I } \\
\text { Fe I } \\
\text { Fe I } ? \\
\text { Fe I } \\
\text { Fe I }\end{array}$ & $\begin{array}{l}3337.915 \\
3330.206 \\
3328.589 \\
3328.470 \\
3324.142\end{array}$ & $\begin{array}{l}1 \\
1 \\
0 \\
1 n \\
0\end{array}$ & $\begin{array}{l}3337.923 \\
3330.234 \\
3328.583 \\
3328.475 \\
3324.150\end{array}$ & $\begin{array}{r}42.3 \\
13.2 \\
2.9 \\
12.2 \\
19.4\end{array}$ & $\begin{array}{l}+0.008 \\
+0.028 \\
-0.006 \\
+0.005 \\
+0.008\end{array}$ & $\begin{array}{c}\mathrm{Fe} \text { I } \\
\mathrm{Fe} \mathrm{I}-\mathrm{NH} \\
\mathrm{Fe} \text { I? } \\
\mathrm{Fe} \text { I } \\
\mathrm{Fe} I ?\end{array}$ \\
\hline $\begin{array}{l}3515.534 \\
3510.682 \\
3506.946 \\
3500.164 \\
3499.271\end{array}$ & $\begin{array}{l}1 \\
2 \\
1 \\
2 \\
1\end{array}$ & $\begin{array}{l}3515.535 \\
3510.685 \\
3506.938 \\
3500.157 \\
3499.269\end{array}$ & $\begin{array}{r}\text { 7. } 7 \\
15.0 \\
4.4 \\
11.1 \\
6.6\end{array}$ & $\begin{array}{l}+0.001 \\
+0.003 \\
-0.008 \\
-0.007 \\
-0.002\end{array}$ & $\begin{array}{l}\text { Fe I } \\
\text { Fe I } \\
\text { Fe I } \\
\text { Fe I } \\
\text { Fe I }\end{array}$ & $\begin{array}{l}3316.838 \\
3316.558 \\
3311.200 \\
3310.916 \\
3298.537\end{array}$ & $\begin{array}{l}0 \\
1 \\
0 n \\
0 n \\
1\end{array}$ & $\begin{array}{l}3316.851 \\
3316.569 \\
3311.215 \\
3310.918 \\
3298.558\end{array}$ & $\begin{array}{r}12.5 \\
11.4 \\
15.4 \\
8.2 \\
14.8\end{array}$ & $\begin{array}{l}+0.013 \\
+0.011 \\
+0.015 \\
+0.002 \\
+0.021\end{array}$ & $\begin{array}{c}\text { Fe I-V II } \\
\text { Fe I } \\
\text { Fe I } \\
\text { Fe I } \\
\text { Fe I }\end{array}$ \\
\hline $\begin{array}{l}3498.755 \\
3488.827 \\
3487.138 \\
3486.142 \\
3483.890\end{array}$ & $\begin{array}{l}2 \\
2 \\
0 \\
1 \\
2\end{array}$ & $\begin{array}{l}3498.749 \\
3488.826 \\
3487.150 \\
3486.143 \\
3483.884\end{array}$ & $\begin{array}{r}13.4 \\
19.5 \\
3.7 \\
10.3 \\
13.4\end{array}$ & $\begin{array}{l}-0.006 \\
-0.001 \\
+0.012 \\
+0.001 \\
-0.006\end{array}$ & $\begin{array}{c}\mathrm{Fe} \text { I } \\
\mathrm{Fe} \mathrm{I} \\
\mathrm{Fe} \mathrm{I} ? \\
\mathrm{Fe} \mathrm{I} \\
\mathrm{Fe} 1\end{array}$ & $\begin{array}{l}3294.621 \\
3282.440 \\
3268.885 \\
3263.062 \\
3262.878\end{array}$ & $\begin{array}{l}0 n \\
1 \\
1 \\
1 \\
1\end{array}$ & $\begin{array}{l}3294.622 \\
3282.447 \\
3268.860 \\
3263.073 \\
3262.902\end{array}$ & $\begin{array}{r}11.4 \\
11.0 \\
5.2 \\
12.9 \\
21.7\end{array}$ & $\begin{array}{l}+0.001 \\
+0.007 \\
-0.025 \\
+0.011 \\
+0.024\end{array}$ & $\begin{array}{c}\text { Fe I } \\
\text { Fe I } \\
\text { Fe I? } \\
\text { Fe I } \\
\text { Fe I }\end{array}$ \\
\hline $\begin{array}{l}3482.446 \\
3472.318 \\
3457.894 \\
3450.743 \\
3448.606\end{array}$ & $\begin{array}{l}1 \\
0 n \\
1 \\
1 \\
1 n\end{array}$ & $\begin{array}{l}3482.451 \\
3472.307 \\
3457.894 \\
3450.747 \\
3448.592\end{array}$ & $\begin{array}{r}10.6 \\
16.4 \\
7.5 \\
2.0 \\
6.7\end{array}$ & $\begin{array}{r}+0.005 \\
-0.011 \\
0.000 \\
+0.004 \\
-0.014\end{array}$ & $\begin{array}{l}\text { Fe I Fe II } \\
\frac{\text { Fe I }}{\mathrm{Fe} \mathrm{I}-} \\
\text { Fe I Ti I } \\
\text { Fe I }\end{array}$ & $\begin{array}{l}3261.801 \\
3261.636 \\
3260.723 \\
3260.460 \\
3259.708\end{array}$ & $\begin{array}{l}0 \\
0 \\
0 \\
0 \\
1\end{array}$ & $\begin{array}{l}3261.817 \\
3261.639 \\
3260.692 \\
3260.472 \\
3259.713\end{array}$ & $\begin{array}{r}16.9 \\
37.2 \\
4.5 \\
4.6 \\
8.6\end{array}$ & $\begin{array}{l}+0.016 \\
+0.003 \\
-0.031 \\
+0.012 \\
+0.005\end{array}$ & $\begin{array}{c}\text { Fe I } \\
\text { Fe I? } \\
\text { Fe I? } \\
\text { Fe I? } \\
\text { Fe I }\end{array}$ \\
\hline $\begin{array}{l}3444.532 \\
3435.219 \\
3430.066 \\
3429.179 \\
3425.441\end{array}$ & $\begin{array}{l}2 \\
0 n \\
0 n \\
1 \\
1\end{array}$ & $\begin{array}{l}3444.518 \\
3435.246 \\
3430.083 \\
3429.148 \\
3425.446\end{array}$ & $\begin{array}{r}\text { 13. } 2 \\
1.0 \\
0.6 \\
1.3 \\
4.7\end{array}$ & $\begin{array}{l}-0.014 \\
+0.027 \\
+0.017 \\
-0.031 \\
+0.005\end{array}$ & $\begin{array}{c}\text { Fe I } \\
\text { Fe I? } \\
\text { Fe I? } \\
\text { Fe I? } \\
\text { Fe I Nb II }\end{array}$ & $\begin{array}{l}3258.092 \\
3249.844 \\
3249.504 \\
3241.378 \\
3232.656\end{array}$ & $\begin{array}{l}1 \\
1 \\
1 \\
1 n \\
1\end{array}$ & $\begin{array}{l}3258.100 \\
3249.861 \\
3249.535 \\
3241.391 \\
3232.687\end{array}$ & $\begin{array}{l}11.4 \\
13.7 \\
25.6 \\
17.9 \\
16.5\end{array}$ & $\begin{array}{l}+0.008 \\
+0.017 \\
+0.031 \\
+0.013 \\
+0.031\end{array}$ & $\begin{array}{c}\mathrm{Fe} \mathrm{I} \\
\mathrm{Fe} \mathrm{I} \\
\mathrm{Fe} \mathrm{I}-\mathrm{VII} \\
\mathrm{Fe} \mathrm{I} \\
\mathrm{Fe} \mathrm{I}-\end{array}$ \\
\hline $\begin{array}{l}3423.558 \\
3422.120 \\
3421.930 \\
3420.250 \\
3418.905\end{array}$ & $\begin{array}{l}1 \\
2 \\
1 \\
0 n \\
1\end{array}$ & $\begin{array}{l}3423.534 \\
3422.127 \\
3421.900 \\
3420.228 \\
3418.881\end{array}$ & $\begin{array}{r}3.2 \\
13.1 \\
0.6 \\
2.9 \\
16.1\end{array}$ & $\begin{array}{r}-0.024 \\
+0.007 \\
-0.030 \\
-0.022 \\
-0.024\end{array}$ & $\begin{array}{c}\mathrm{NH} \text { - Fe I? } \\
\text { Fe I } \\
\text { Fe I } \\
\text { Fe I? } \\
\text { Fe I }\end{array}$ & $\begin{array}{l}3223.480 \\
3216.343 \\
3205.782 \\
3204.454 \\
3204.306\end{array}$ & $\begin{array}{l}1 n \\
0 \\
1 \\
0 n \\
1\end{array}$ & $\begin{array}{l}3223.449 \\
3216.359 \\
3205.783 \\
3204.453 \\
3204.284\end{array}$ & $\begin{array}{r}17.9 \\
1.7 \\
12.2 \\
4.7 \\
17.0\end{array}$ & $\begin{array}{l}-0.031 \\
+0.016 \\
+0.001 \\
-0.001 \\
-0.022\end{array}$ & $\begin{array}{c}\text { Fe I } \\
\text { Fe I? } \\
\text { Fe I } \\
\text { Fe I } \\
- \text { Fe I }\end{array}$ \\
\hline $\begin{array}{l}3416.840 \\
3414.432 \\
3408.474 \\
3401.007 \\
3400.662\end{array}$ & $\begin{array}{l}1 \\
0 \\
1 \\
1 \\
1\end{array}$ & $\begin{array}{l}3416.869 \\
3414.403 \\
3408.505 \\
3400.987 \\
3400.645\end{array}$ & $\begin{array}{r}\text { 6. } 1 \\
4.7 \\
6.5 \\
12.2 \\
10.3\end{array}$ & $\begin{array}{r}+0.029 \\
-0.029 \\
+0.031 \\
-0.020 \\
-0.017\end{array}$ & $\begin{array}{c}\text { NH Fe I } \\
\text { Fe I? } \\
\text { Fe I } \\
\text { Fe I } \\
\text { Fe I }\end{array}$ & $\begin{array}{l}3202.958 \\
3198.492 \\
3195.968 \\
3195.235 \\
3192.521\end{array}$ & $\begin{array}{l}2 \\
1 \\
1 \\
1 n \\
2\end{array}$ & $\begin{array}{l}3202.942 \\
3198.487 \\
3195.990 \\
3195.230 \\
3192.534\end{array}$ & $\begin{array}{r}\text { 4. } 7 \\
\text { 18. } 2 \\
\text { 32. } 2 \\
\text { 26. } 1 \\
\text { 31. } 9\end{array}$ & $\begin{array}{l}-0.016 \\
-0.005 \\
+0.022 \\
-0.005 \\
+0.013\end{array}$ & $\begin{array}{c}\mathrm{Fe} \mathrm{I} \\
\mathrm{Fe} \mathrm{I} \\
\mathrm{Fe} \mathrm{I} \\
\mathrm{Fe} I \\
\mid \mathrm{Fe} \mathrm{I}-\mathrm{CH} ?\end{array}$ \\
\hline $\begin{array}{l}3398.620 \\
3384.946 \\
3384.392 \\
3381.990 \\
3381.498\end{array}$ & $\begin{array}{l}1 \\
0 n \\
0 \\
1 n \\
1 n\end{array}$ & $\begin{array}{l}3398.612 \\
3384.925 \\
3384.425 \\
3381.993 \\
3381.495\end{array}$ & $\begin{array}{r}11.8 \\
3.5 \\
5.0 \\
11.2 \\
0.9\end{array}$ & $\begin{array}{l}-0.008 \\
-0.021 \\
+0.033 \\
+0.003 \\
-0.003\end{array}$ & $\begin{array}{c}\mathrm{Fe} \text { I } \mid \mathrm{Ti} \text { I } \\
\mathrm{Fe} \text { I } \\
\mathrm{Fe} \text { I? } \\
\mathrm{Fe} \text { I } \\
\text { Co I Fe I }\end{array}$ & $\begin{array}{l}3189.612 \\
3186.276 \\
3184.215 \\
3181.142 \\
3170.978\end{array}$ & $\begin{array}{l}0 \\
0 \\
1 \\
0 n \\
0\end{array}$ & $\begin{array}{l}3189.634 \\
3186.272 \\
3184.210 \\
3181.131 \\
3170.985\end{array}$ & $\begin{array}{r}0.5 \\
9.6 \\
17.6 \\
4.1 \\
14.7\end{array}$ & $\begin{array}{l}+0.022 \\
-0.004 \\
-0.005 \\
-0.011 \\
+0.007\end{array}$ & $\begin{array}{l}\text { Fe I } \\
\text { Fe I } \\
\text { Fe I } \\
\text { Fe I } \\
\text { Fe } 1 \text { ? }\end{array}$ \\
\hline $\begin{array}{l}3381.132 \\
3380.756 \\
3379.688 \\
3377.971 \\
3377.345\end{array}$ & $\begin{array}{l}1 \\
1 \\
1 \\
3 \\
0 n\end{array}$ & $\begin{array}{l}3381.132 \\
3380.752 \\
3379.706 \\
3377.977 \\
3377.361\end{array}$ & $\begin{array}{r}16.1 \\
30.9 \\
0.9 \\
23.1 \\
1.8\end{array}$ & $\begin{array}{r}0.000 \\
-0.004 \\
+0.018 \\
+0.006 \\
+0.016 \\
+0.006\end{array}$ & $\begin{array}{l}\text { Fe I- } \\
\text { Sr II-Fe I } \\
\text { Fe I } \\
\text { Fe I } \\
\text { Fe I-VI }\end{array}$ & $\begin{array}{l}3165.280 \\
\\
3159.437 \\
3158.193 \\
3157.293 \\
3146.270\end{array}$ & $\begin{array}{l}0 \\
\\
1 \\
0 \\
1 \\
1\end{array}$ & $\begin{array}{l}3165.266 \\
3159.436 \\
3158.191 \\
3157.294 \\
3146.301\end{array}$ & $\begin{array}{c}21.9 \\
\text { Est. Int. } \\
{[-1]} \\
{[-1]} \\
{[0]} \\
{[0]}\end{array}$ & $\begin{array}{l}-0.014 \\
-0.001 \\
-0.002 \\
+0.001 \\
+0.031\end{array}$ & $\begin{array}{l}\text { Fe I } \\
\text { Fe I } \\
\text { Fe I? } \\
\text { Fe I } \\
\text { Fe I }\end{array}$ \\
\hline $\begin{array}{l}3373.300 \\
3371.304 \\
3368.800 \\
3367.660\end{array}$ & $\begin{array}{l}1 \\
1 \\
1 n \\
1\end{array}$ & $\begin{array}{l}3373.316 \\
3371.295 \\
3368.821 \\
3367.677\end{array}$ & $\begin{array}{l}17.1 \\
18.1 \\
16.5 \\
17.2\end{array}$ & $\begin{array}{l}+0.000 \\
+0.016 \\
-0.009 \\
+0.021 \\
+0.017\end{array}$ & $\begin{array}{l}\text { Fe I } \\
\text { Fe I } \\
\text { Fe I } \\
\text { NH Fe I }\end{array}$ & $\begin{array}{l}3139.485 \\
3135.590 \\
3134.401 \\
3132.660 \\
3123.545\end{array}$ & $\begin{array}{l}1 \\
1 n \\
1 \\
1 \\
1 n\end{array}$ & $\begin{array}{l}3139.486 \\
3135.589 \\
3134.396 \\
3132.635 \\
3123.561\end{array}$ & $\begin{array}{r}{[-1]} \\
{[0]} \\
{[1]} \\
{[1]} \\
{[1]}\end{array}$ & $\begin{array}{l}+0.001 \\
-0.001 \\
-0.005 \\
-0.025 \\
+0.016\end{array}$ & $\begin{array}{c}\text { Fe I } \\
\text { Fe I } \\
\text { Fe I? } \\
\text { Fe I } \\
\text { Fe I }\end{array}$ \\
\hline $\begin{array}{l}3367.292 \\
3364.402 \\
3357.558 \\
3354.512 \\
3340.184\end{array}$ & $\begin{array}{l}1 \\
1 \\
1 \\
1 \\
0\end{array}$ & $\begin{array}{l}3367.299 \\
3364.400 \\
3357.569 \\
3354.537 \\
3340.178\end{array}$ & $\begin{array}{r}8.9 \\
10.1 \\
11.6 \\
7.3 \\
3.7\end{array}$ & $\begin{array}{l}+0.007 \\
-0.002 \\
+0.011 \\
+0.025 \\
-0.006\end{array}$ & $\begin{array}{l}\text { Fe I } \\
\text { Fe I NH } \\
\text { Fe I } \\
\text { Fe I? Ti II? } \\
\text { Fe I }\end{array}$ & $\begin{array}{l}3120.220 \\
3116.502 \\
3115.862 \\
3115.656 \\
3109.614\end{array}$ & $\begin{array}{l}2 n \\
1 n \\
1 \\
2 \\
1 n\end{array}$ & $\begin{array}{l}3120.237 \\
3116.503 \\
3115.883 \\
3115.668 \\
3109.622\end{array}$ & $\begin{array}{l}{[1]} \\
{[1]} \\
{[0]} \\
{[1]} \\
{[1]}\end{array}$ & $\begin{array}{l}+0.017 \\
+0.001 \\
+0.021 \\
+0.012 \\
+0.00 \varepsilon\end{array}$ & $\begin{array}{c}\text { Fe I } \\
\text { Fe I } \\
\text { Fe I } \\
\text { Fe I Cr II } \\
\text { Fe I }\end{array}$ \\
\hline
\end{tabular}


TABLE 5.-Faint Lines of Fe I in the solar spectrum-Concluded

\begin{tabular}{|c|c|c|c|c|c|c|c|c|c|c|c|}
\hline \multicolumn{2}{|c|}{ Laboratory } & \multicolumn{4}{|c|}{ Sun } & \multicolumn{2}{|c|}{ Laboratory } & \multicolumn{4}{|c|}{ Sun } \\
\hline $\begin{array}{c}\text { Wave- } \\
\text { length } \\
\text { A }\end{array}$ & $\begin{array}{c}\text { Inten- } \\
\text { sity }\end{array}$ & $\begin{array}{l}\text { Wave- } \\
\text { length } \\
\text { A }\end{array}$ & $\begin{array}{l}\text { Inten- } \\
\text { sity } \\
\Delta \lambda / \lambda\end{array}$ & $\odot_{\mathrm{A}}^{- \text {Lab. }}$ & $\begin{array}{l}\text { Solar } \\
\text { Indentifi- } \\
\text { cation }\end{array}$ & $\begin{array}{l}\text { Wave- } \\
\text { length } \\
\text { A }\end{array}$ & $\begin{array}{c}\text { Inten- } \\
\text { sity }\end{array}$ & $\begin{array}{l}\text { Wave- } \\
\text { length } \\
\text { A }\end{array}$ & $\begin{array}{l}\text { Inten- } \\
\text { sity } \\
\Delta \lambda / \lambda\end{array}$ & $\odot$ - Lab. & $\begin{array}{l}\text { Solar } \\
\text { Identifi- } \\
\text { cation }\end{array}$ \\
\hline 3107.322 & $1 n$ & 3107. 322 & {$[0]$} & 0.000 & Fe I & 2985. 750 & 0 & 2985. 73 & [1] & -0.02 & $\mathrm{Fe}_{1}$ \\
\hline 3098. 963 & 1 & 3098. 968 & {$[-1]$} & +0.005 & Fe I & 2979. 867 & 0 & 2979. 88 & [1] & +0.01 & Fe I \\
\hline 3087.420 & $1 n$ & 3087.453 & {$[0]$} & +0.033 & $\mathrm{Fe}$ I OH & 2978. 060 & 1 & 2978. 055 & [3] & -0.005 & $\mathrm{Fe} 1$ \\
\hline 3081.278 & 1 & 3081. 247 & [2] & -0.031 & $\mathrm{OH} \mathrm{Fe} \mathbf{I}$ & 2975. 298 & 0 & 2975. 278 & [3] & -0.020 & $-\mathrm{Fe}_{\mathrm{I}}$ \\
\hline 3049.564 & $1 n$ & 3049.546 & {$[2]$} & -0.018 & Fe I & 2971. 776 & 0 & 2971.77 & [1] & -0.01 & $\mathrm{Fe}_{\mathrm{I}}$ \\
\hline 3049.356 & $1 n$ & 3049. 349 & [3] & -0.007 & $\mathrm{Fe} I$ & 2963.518 & $0 n$ & 2963.52 & {$[3 \mathrm{~N}]$} & 0.00 & $\mathrm{Fe}$ 1? $\mid \mathrm{Cr}$ II \\
\hline 3038. 334 & $0 n$ & 3038. 312 & [3] & -0.022 & $\mathrm{Fe} \mathrm{I}$ & 2962. 585 & $1 n$ & 2962.59 & {$[-1]$} & 0.00 & Fe I \\
\hline 3012.942 & $1 n$ & 3012. 937 & [3] & -0.005 & Fe I & 2958. 462 & 1 & 2958.45 & {$[0 \mathrm{~N}]$} & -0.01 & Fe I \\
\hline 3011.883 & 2 & 3011.88 & {$[0 \mathrm{~N}]$} & 0.00 & Fe I & 2947. 116 & 0 & 2947. 04 & {$[-1]$} & -0.08 & $\mathrm{~W}_{\mathrm{I}}-\mathrm{Fe} \mathrm{I} ?$ \\
\hline \multirow[t]{2}{*}{ 2995. 256} & 0 & 2995. 260 & {$[3]$} & +0.004 & $\mathrm{Fe} \mathrm{I}$ & 2946. 095 & 1 & 2946. 08 & {$[-1]$} & +0.02 & $\mathrm{Fe}_{\mathrm{I}}$ \\
\hline & & & & & & 2945. 702 & 1 & 2945. 65 & {$[-1]$} & -0.05 & Fe I \\
\hline
\end{tabular}

(Paper 65A1-80) 